\title{
EVALUATION OF ENDOTHELIAL CELL RESPONSES TO ELEVATED GLUCOSE IN VITRO
}

\author{
A Thesis \\ presented to \\ the Faculty of California Polytechnic State University, \\ San Luis Obispo \\ In Partial Fulfillment \\ Of the Requirements for the Degree \\ Master of Science in Biomedical Engineering \\ By \\ Gabriella Paige Byrd Sugerman \\ August 2018
}


(C) 2018

Gabriella Paige Byrd Sugerman

ALL RIGHTS RESERVED 


\section{COMMITTEE MEMEBERSHIP}

TITLE: Evaluation of Endothelial Cell Responses to Elevated Glucose In Vitro

AUTHOR: Gabriella Paige Byrd Sugerman

DATE SUBMITTED: August 2018

COMMITTEE CHAIR: $\quad$ Kristen O'Halloran Cardinal, Ph.D.

Professor of Biomedical Engineering

COMMITTEE MEMBER: $\quad$ Trevor Ryan Cardinal, Ph.D.

Professor of Biomedical Engineering

COMMITTEE MEMBER: Daniel G. Peterson, Ph.D.

Professor of Animal Science 


\begin{abstract}
Evaluation of Endothelial Cell Responses to Elevated Glucose In Vitro
\end{abstract}

Gabriella Paige Byrd Sugerman

Developing a tissue-engineered Blood Vessel Mimic (BVM) to represent diabetic macrovascular disease could expedite design of new vascular devices specifically tailored to diabetic patients. In contribution toward this model, this thesis assessed Human Umbilical Vein Endothelial Cell (HUVEC) responses to high glucose conditions. Interleukin 6 (IL-6) and Cluster of Differentiation 36 (CD36) were selected to signify oxidative stress activity, a hallmark of diabetic macrovascular disease. Next, activity of potential reference genes B2M, HPRT1, and ACTB was assessed. All genes were found to exceed acceptable variability, so the $\mathrm{E}^{-\Delta \mathrm{C}} \mathrm{T}$ method of data analysis was selected. Next, cellular responses to high glucose treatment at $10.5 \mathrm{mM}$ glucose and $25.5 \mathrm{mM}$ glucose for 7 and 14 days were measured by qPCR. IL-6 mRNA expression increased significantly $(\mathrm{p}<0.001)$ following treatment with $25.5 \mathrm{mM}$ glucose at both timepoints.

Finally, fluorescent staining for Reactive Oxygen Species (ROS) production and cell viability was performed on HUVECs treated with 10.5- and 25.5-mM glucose for 24 and 48 hours. No differences in ROS production or cell viability were detected due to uncontrolled cell damage during the two-hour staining and imaging procedure. This thesis was limited by low reaction efficiency in qPCR reactions due to mistaken purchasing of primers with included probe-quencher reporters. Measurement of reaction efficiency facilitated valid analysis of data collected using these primers. Imaging experiments were unsuccessful due to a lack of incubation equipment designated for cells undergoing live staining and imaging. Alternative imaging assessments of oxidative stress activity were proposed to circumvent this problem. 


\section{ACKNOWLEDGMENTS}

I would like to thank Dr. Kristen Cardinal for her guidance and support throughout my pursuit of a Master of Science degree. The privilege of working with Dr. Cardinal has inspired my curiosity, determination, and enduring love of research. Her positive impact on all of her students—especially those in her research laboratory—inspires greatness in our department.

I would also like to thank my committee members, Dr. Trevor Cardinal and Dr. Daniel Peterson, for their time and expertise. Additionally, I would like to thank my fellow research lab members, especially Enoch Tsui and Conor Hedigan, for their assistance in the laboratory, willingness to share their knowledge, and moral support.

Finally, I would like to thank my family—Mom, Dad, Sam, Doris, and Liz—for their unending support and love. 


\section{TABLE OF CONTENTS}

\section{Page}

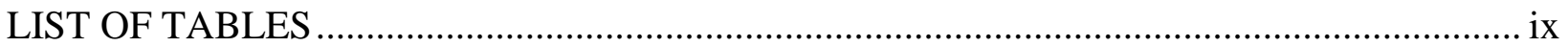

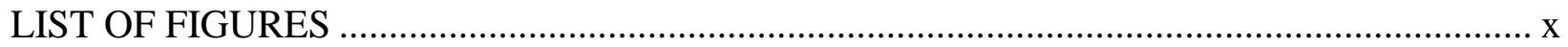

\section{CHAPTER}

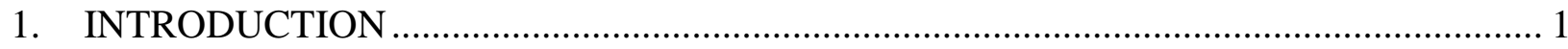

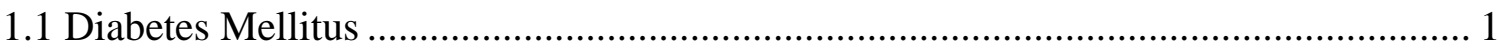

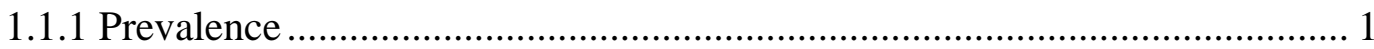

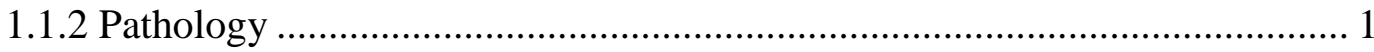

1.2 Traditional Device Testing Progression............................................................ 5

1.3 Blood Vessel Mimics ........................................................................................ 6

1.3.1 Inducing Elevated Glucose Conditions in BVMs .................................. 8

1.4 Cellular Responses and Expression of mRNA and Proteins .................................. 9

1.4.1 Cellular Response to Oxidative Stress and qPCR .................................... 9

1.4.2 Reactive Oxygen Species and Fluorescent Microscopy ............................ 14

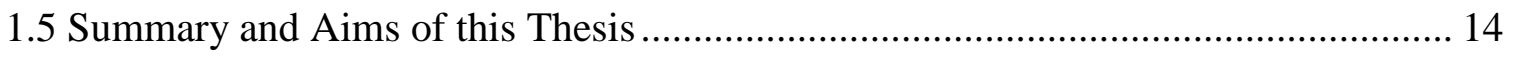

\section{DETERMINING GENES OF INTEREST AND OPTIMIZING QPCR DATA ANALYSIS}

16

2.1: Introduction 16

2.2: Gene Selection and Primer Acquisition 16 
2.3 Data Analysis Methods

2.4 Data Analysis Results ......................................................................................... 29

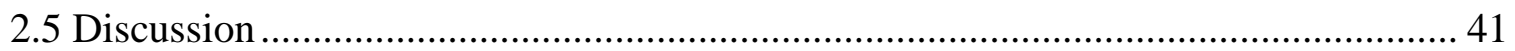

3. EVALUATING GENE EXPRESSION IN RESPONSE TO INCREASED

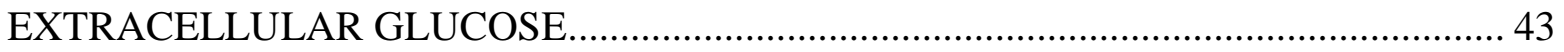

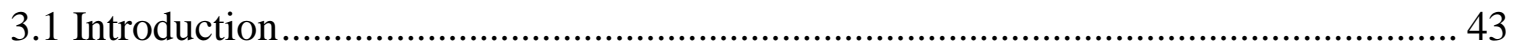

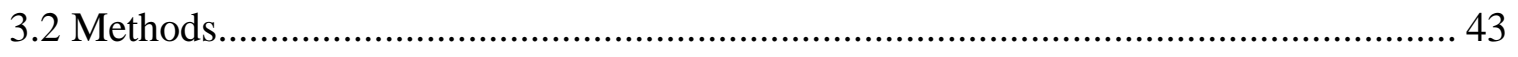

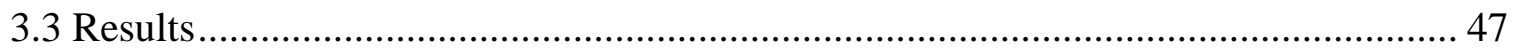

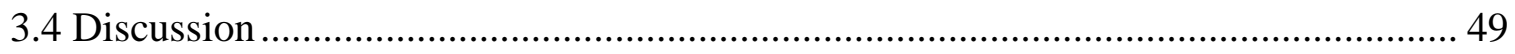

4. EVALUATING ROS GENERATION IN RESPONSE TO INCREASED

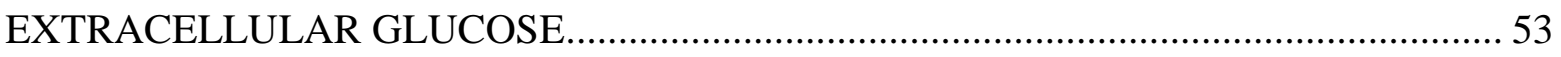

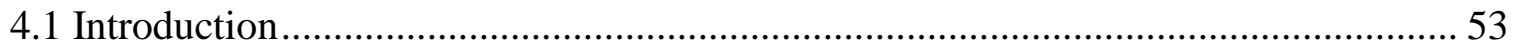

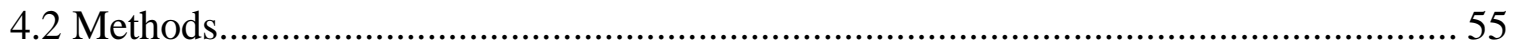

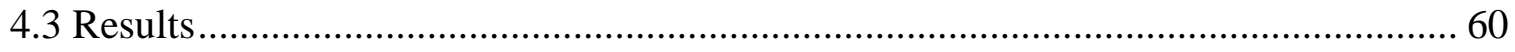

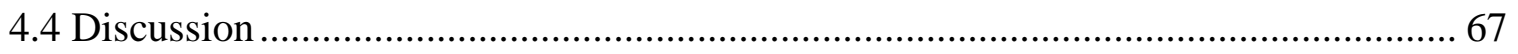

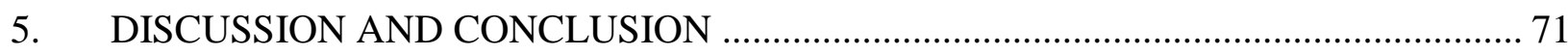

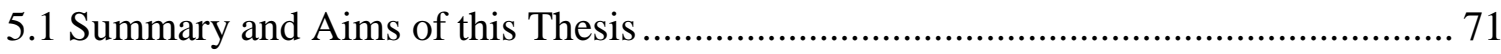

5.2 Challenges and Limitations............................................................................... 72

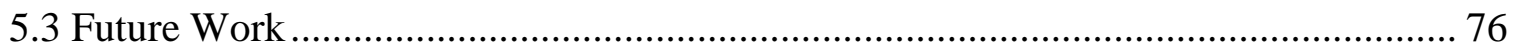

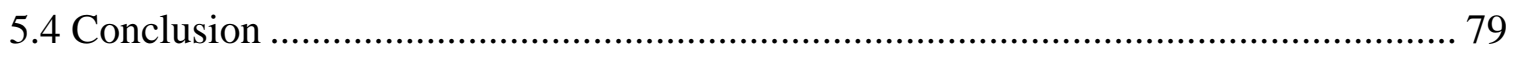




\section{APPENDICES}

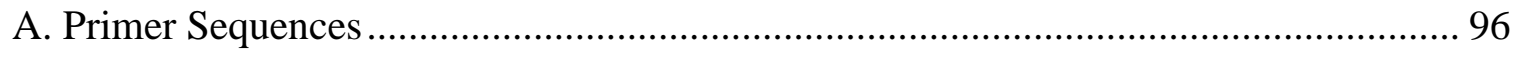

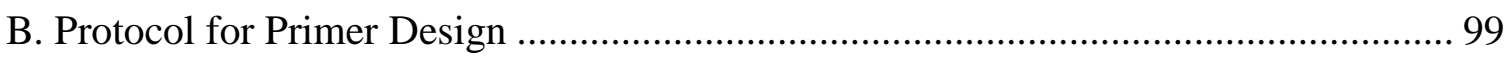

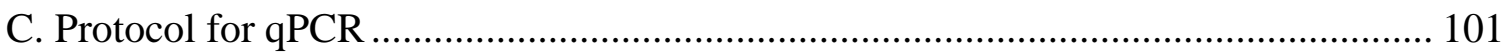

D. Protocol for qPCR Data Analysis .......................................................................... 104

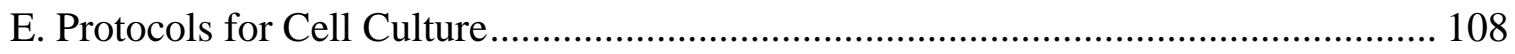

F. Protocols for RNA Isolation and Purity Assessment............................................... 110

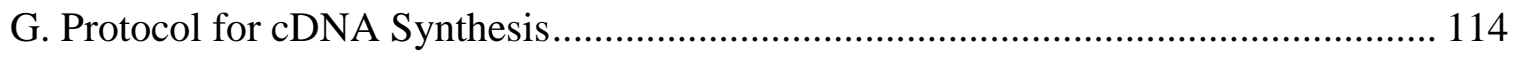

H. Protocols for Cell Culture for Confocal Microscopy ……………............................. 116

I. Protocols for ROS Production and Cell Viability Assessment..................................... 118

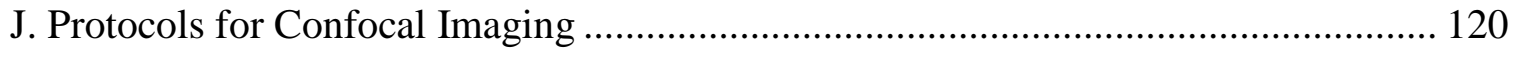

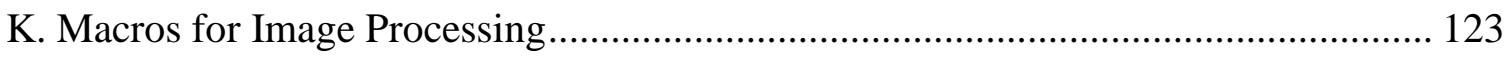

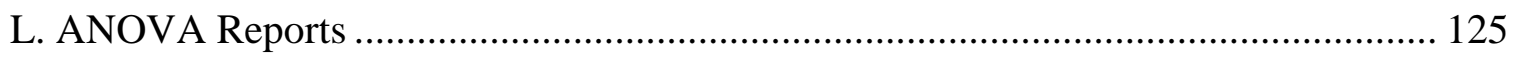

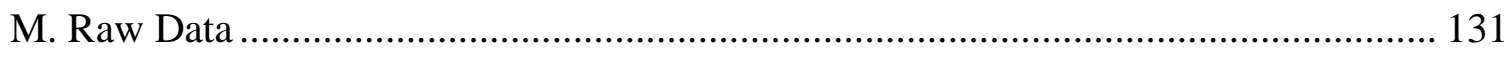




\section{LIST OF TABLES}

Table

Page

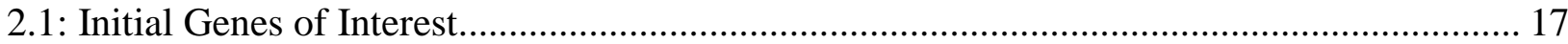

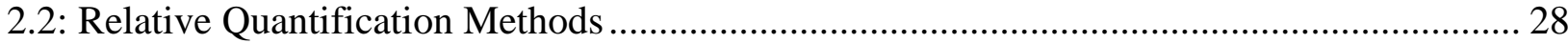

2.3: Measured Efficiencies by Gene from Standard Curve Analysis ..................................... 31

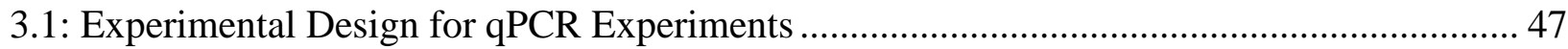

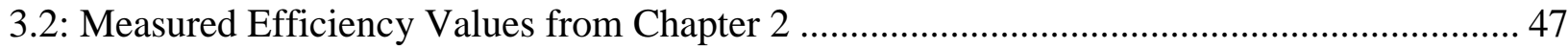

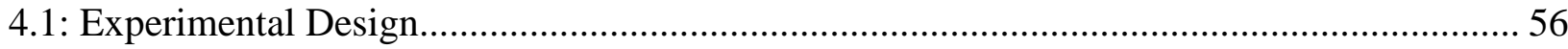

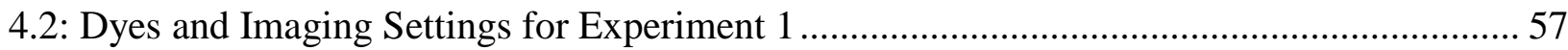

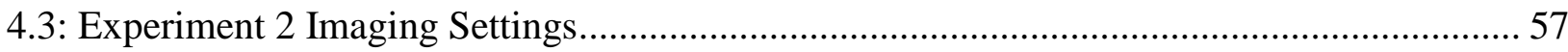

4.4: Summary of Statistical Significance of Imaging Findings ............................................ 67 


\section{LIST OF FIGURES}

Figure $\quad$ Page

1.1: Insulin Resistance Increases with Disease State. Progression of severity of insulin resistance and the associated diagnoses with their associated macrovascular symptoms [5]

1.2: Decreased Event-Free Survival in Diabetic Patients with Vascular Stents [13]. Adverse events include death, cardiopulmonary events (stroke, myocardial infarction, pulmonary

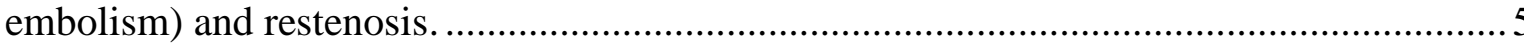

1.3: Blood Vessel and BVM Layers. Blood vessels are composed of tunicae intima, media, and adventitia, while BVMs are composed of polymer scaffolds and cell linings. 7

1.4: Bioreactor Schematic in the Cal Poly Tissue Engineering lab. Cells are sodded into polymer scaffold before incorporation into peristaltic flow system. Cell media is pushed through tubing, through the lumen of the BVM, circulating as cell layer develops. Stopcocks isolate flow through transmural or transluminal paths.

1.5: Pathways Involved in Hyperglycemia-Induced Endothelial Damage. Many pathways interact to initiate diabetic macrovascular disease. Both targets for gene expression analysis are mechanistically understood to be upregulated under hyperglycemic conditions [8].

2.1: Amplification Plot of All Initial Primers. Each line represents one individual qPCR sample. The x-axis represents cycle number, or progress of the qPCR, while Delta Rn on the $\mathrm{y}$-axis represents change in fluorescence intensity. The Delta $\mathrm{Rn}$ value is the derivative of fluorescence intensity. Sample A shows irregular replication while Samples in Region B failed to replicate. 
2.2: Amplification Plot with Linear Amplification Region, Threshold, and $\mathrm{C}_{\mathrm{T}}$.

2.3: Amplification Plots of Successful Initial Primers. Isolated here are only those primers which produced three CT values approximately equal to one another. As in Figure 2.2, the $\mathrm{x}$-axis represents PCR progression and the $\mathrm{y}$-axis represents change in fluorescence intensity. Each line represents one sample qPCR well

2.4: Amplification Curves of PrimeTime Preverified Primers (A)IL-6 (B)ACTB (C)B2M (D)HPRT1 (E)CD36. ACTB (B) displays greater variability among replicates than other sets.

2.5: Dissociation curves of (A) ACTB and (B) IL-6. ACTB has a peak in the non-template control sample, demonstrating primer-dimer formation

2.6: Standard Curve Amplification Plots: Change in fluorescence intensity over time (Delta $\mathrm{Rn}$ ) versus progression of qPCR (Cycle Number) for (A) IL-6 (B) CD36 (C) B2M and (D) HPRT1 pre-verified primers. Horizontal lines depict threshold values.

2.7: Representative Standard Curve Analysis. B2M cDNA dilution factor versus average

$\mathrm{C}_{\mathrm{T}}$ value. Slope of the trendline is used to determine qPCR efficiency

2.8: $\mathrm{E}^{-\mathrm{C}} \mathrm{T}$ Analysis. (A) $\mathrm{B} 2 \mathrm{M}$ expression decreases with Moderate treatment and increases with High glucose treatment. (B) CD36 expression remains consistent across treatments. (C) HPRT1 expression decreases with Moderate treatment. (D) IL-6 expression increases with High glucose treatment. All values are in arbitrary units

presented as mean \pm SEM.

2.9: $\mathrm{E}^{-\Delta \mathrm{C}}{ }_{\mathrm{T}}$ Analysis of 7-Day Data. (A) B2M (B) CD36 (C) HPRT1 and (D) IL-6 expression all increased in High glucose condition compared to Moderate glucose condition relative to Basal. All values are in arbitrary units presented as mean \pm SEM. 
2.10: $2^{-\Delta \Delta C}$ T Analysis of 7-Day Data. Influence of reference gene on results of comparative $\mathrm{C}_{\mathrm{T}}$ method. Expression of genes (A, C, E) relative to HPRT1 expression and (B, D, F) relative to B2M expression. All values are in arbitrary units presented as mean \pm SEM.... 37

2.11: Probe-Quencher qPCR Reporting Mechanism ..................................................................39

2.12: Probe-Quencher Reporting of 7-Day Responses. Data are presented as mean \pm SEM ... 40

3.1: Relative Abundance Comparisons Between Assumed and Calculated Efficiency Values after 7 Day Treatment. (A)B2M expression increased at High glucose concentration. (B) CD36 expression remained consistent across treatment groups. (C) HPRT1 expression remained consistent across treatment groups. (D) IL-6 expression increased at High glucose concentration. Data are presented as mean \pm SEM. 48

3.2: Relative Abundance of Target Genes at 14-day Timepoint. (A) B2M expression increased with High glucose treatment. (B) CD36 expression was unaffected by treatment. (C) HPRT1 expression decreased with Moderate glucose treatment but remained consistent from Basal to High glucose treatment. (D) IL-6 expression increased with High glucose treatment. All data are in arbitrary units and are presented as mean \pm SEM.

4.1: Increased Extracellular Glucose Increases ROS Generation and Subsequent Tissue Damage [35]. Glucose transport initiates glycolysis which increases oxidant generation. Reactive Oxygen Species (ROS) are a byproduct of oxidant generation and induce tissue damage by increasing Advanced Glycation End-products (AGEs) and upregulating the PKC pathway. 
4.2: Viability by Treatment and Duration. In Basal $(5.5 \mathrm{mM})$ glucose condition, viability increased between 24 and 48 hours. Neither Moderate $(10.5 \mathrm{mM})$ nor High $(25.5 \mathrm{mM})$ glucose condition displayed change in viability between 24 and 48 hours.

4.3: Representative Images from Experiment 1. (A) Hoechst 33342-positive (B) CarboxyH2DCFDA-positive (C) EthD-1-positive and (D) Composite images of basal (5.5 mM glucose) treatment condition after 48 hours

4.4: ROS Generation as Mean Gray Value by Treatment and Duration. Samples were treated with Basal (5.5 mM), Moderate (10.5 mM), or High (25.5 mM) glucose. Positive Control samples received Basal glucose treatment and TBHP treatment for ROS generation

4.5: Cell Viability by Treatment at 48 Hours. Positive control samples were cultured with basal (5.5 mM) glucose before TBHP treatment which may decrease cell viability. Basal (5.5 mM), Moderate $(10.5 \mathrm{mM})$ and High $(25.5 \mathrm{mM})$ glucose treatments show no effect on cell viability. 64

4.6: Representative Images from Experiment 2. (A) Hoechst 33342-positive (B) CarboxyH2DCFDA-positive (C) EthD-1-positive and (D) Composite images of basal (5.5 mM glucose) treatment condition after 48 hours

4.7: ROS Generation on (A) Day $1(\mathrm{n}=4$ per group) and (B) Day 2 ( $\mathrm{n}=4$ per group) of Experiment 2. Samples were treated with Basal (5.5 mM), Moderate (10.5 mM), or High (25.5 mM) glucose. Positive Control samples received Basal glucose treatment and TBHP treatment for ROS generation. 


\section{INTRODUCTION}

\subsection{Diabetes Mellitus}

\subsubsection{Prevalence}

Diabetes is extremely prevalent in the United States, and incidence is on the rise. The Centers for Disease Control release an annual National Diabetes Statistics Report and estimate that in $2015,9.4 \%$ of the U.S. population—over 30 million people—had diabetes [1]. Of those, 95\% had Type 2 Diabetes, which is primarily caused by lifestyle factors [1]. Insulin resistance and associated endothelial dysfunction often begin many years before diagnosis of Type 2 Diabetes and is increasing among normoglycemic individuals, with estimates of insulin resistance reaching $32.2 \%$ in 1999-2002 [2].

\subsubsection{Pathology}

Diabetes Mellitus is a metabolic disease wherein glucose is improperly processed by the body, potentially leading to widespread tissue damage. There are two distinct pathologies that differ according to which mechanism of insulin-mediated glucose uptake that is dysfunctional. Type 1 Diabetes is characterized by autoimmune destruction of insulin-producing pancreatic $\beta$ cells, leading to negligible blood insulin concentrations. Type 2 Diabetes is characterized by resistance to insulin signaling, leading to an elevated blood insulin concentration [3]. Though the two diseases share many symptoms, including endothelial dysfunction and macrovascular disease, this thesis focuses on the more common and preventable Type 2 Diabetes [1].

In normoglycemic patients, cellular glucose uptake occurs through two balanced mechanisms. Insulin-mediated glucose transport occurs through the cell membrane protein GLUT4 on adipose and muscle cells and non-insulin-mediated glucose transport occurs on all cells through GLUT1. When insulin resistance develops, transport using GLUT4 decreases and 
the mass balance effect leads to increased GLUT1 utilization. One byproduct of glucose metabolism through GLUT1 is hexosamine, which has a negative feedback effect on GLUT4 and exacerbates development of insulin resistance [4]. Decreased insulin utilization leads to hyperinsulinemia, a hallmark of Type 2 Diabetes which is absent in Type 1 Diabetes.

When a molecule of glucose enters a cell, it undergoes glycolysis initiated by hexokinase in the cytosol and is converted to two three-carbon molecules of pyruvate. Pyruvate is transported to the mitochondria where the citric acid cycle, also known as the Kreb's Cycle, converts it to acetyl-CoA. This cycle yields energy in the form of adenosine triphosphate (ATP) and byproducts $\mathrm{NADH}$ and $\mathrm{FADH}_{2}$, which initiate the electron transport chain along the mitochondrial membrane. The electron transport chain converts high-energy NADH and $\mathrm{FADH}_{2}$ molecules to additional ATP [5]. In Type 2 Diabetes, excess glucose flux causes insufficient hexokinase enzyme availability and additional glucose enters the polyol pathway, leading to increased sorbitol production, which induces oxidative stress. Additionally, NADPH is depleted, producing an increased NADH/NAD+ ratio which further contributes to oxidative stress [6].

Oxidative stress caused by hyperglycemia can cause several coincident conditions in multiple organ systems. An increased amount of circulating glucose is widely understood to trigger mitochondrial production of reactive oxygen species (ROS). These oxygen-containing molecules include radical electrons, allowing them to bind essential molecules like DNA and proteins, thereby worsening oxidative stress conditions in the cells [7]. This activates a continuous inflammatory response, leading to systemic damage over long periods of time, including degradation of the nervous and circulatory systems [8].

Figure 1.1 summarizes how symptoms progress as insulin resistance increases, and how hyperinsulinemia progresses in diagnosis to Type 2 Diabetes [8]. Circulating glucose also causes 
cellular damage by creating advanced glycation end products (AGEs), protein and lipid molecules modified by exposure to glucose [9]. AGEs are influential cellular signals, initiating vascular constriction and vascular stiffening that results in systemic atherosclerosis [10]. In Type 2 Diabetes, vascular stiffening manifests in the entire body from retinopathy to peripheral neuropathy, but the highest mortality rate is caused directly by cardiac macrovascular disease [1].

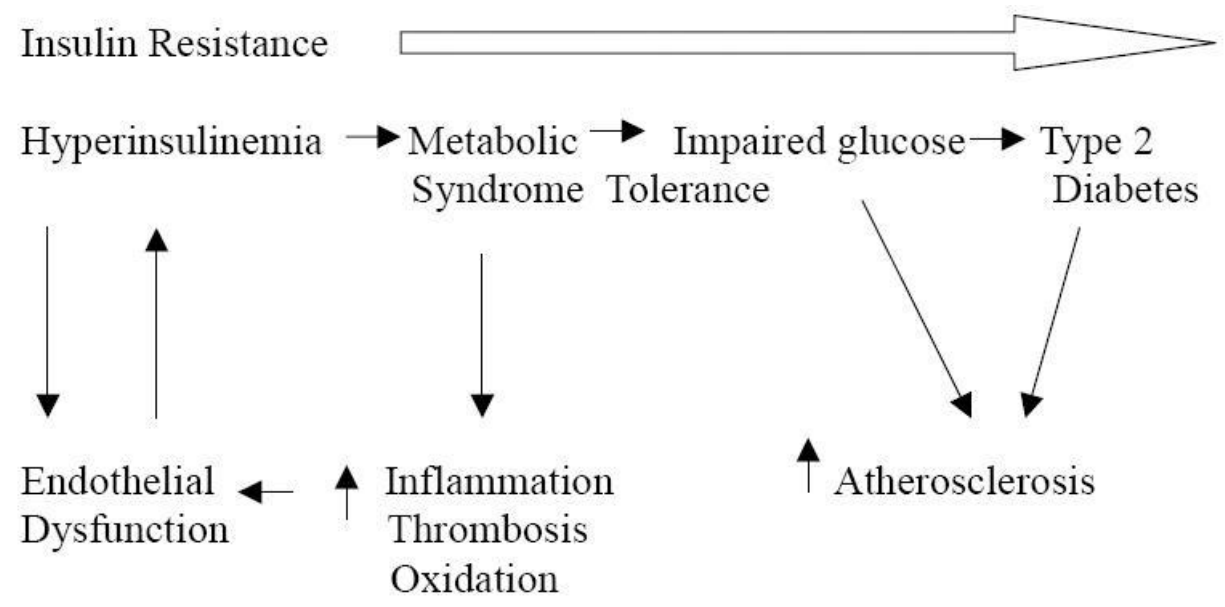

Figure 1.1: Insulin Resistance Increases with Disease State. Progression of severity of insulin resistance and the associated diagnoses with their associated macrovascular symptoms [5]. Macrovascular disease evolves slowly over an individual's lifetime. As chronic stiffening and occlusion of blood vessels, or atherosclerosis, progresses over time, symptoms penetrate from the innermost to outermost layers of blood vessels. The innermost lining of blood vessels is composed of endothelial cells (ECs), which undergo glucose-sensitive signaling pathways in response to oxidative stress. In healthy endothelial cells, insulin signaling leads to phosphorylation of endothelial nitric oxide synthase (eNOS), which increases nitric oxide production [11]. This process becomes dysfunctional as insulin resistance develops, with significant downregulation of insulin receptor substrate 1 (Irs1) and eNOS [12]. In a mouse model using overexpression of a dominant negative endothelium-specific insulin receptor 
(ESMIRO), endothelial insulin resistance has been shown to decrease vasorelaxation responses to acetylcholine, increase superoxide production, and promote development of atherosclerotic lesions $[11,13]$. Endothelial cells transmit insulin from the bloodstream to skeletal muscle, a primary location for insulin-mediated glucose metabolism, so when endothelial insulin signaling is disrupted, glucose uptake by skeletal muscles is decreased [12] and hyperglycemia is aggravated.

These pathways promote inflammation and, in combination with AGE signaling, create a pro-atherosclerotic environment within vessels $[3,8]$. Atherosclerosis is traditionally treated with angioplasty or stenting, procedures intended to increase the inner diameter of the blood vessel by compressing plaques [14]. Due to chronic pro-inflammatory conditions present in diabetes, historically these procedures have a lower rate of success in diabetic patients than in non-diabetic conditions $[4,10-13]$. Figure 1.2 depicts the increase in adverse outcomes like death, cardiac events, and repeat procedures among diabetic patients [17]. 


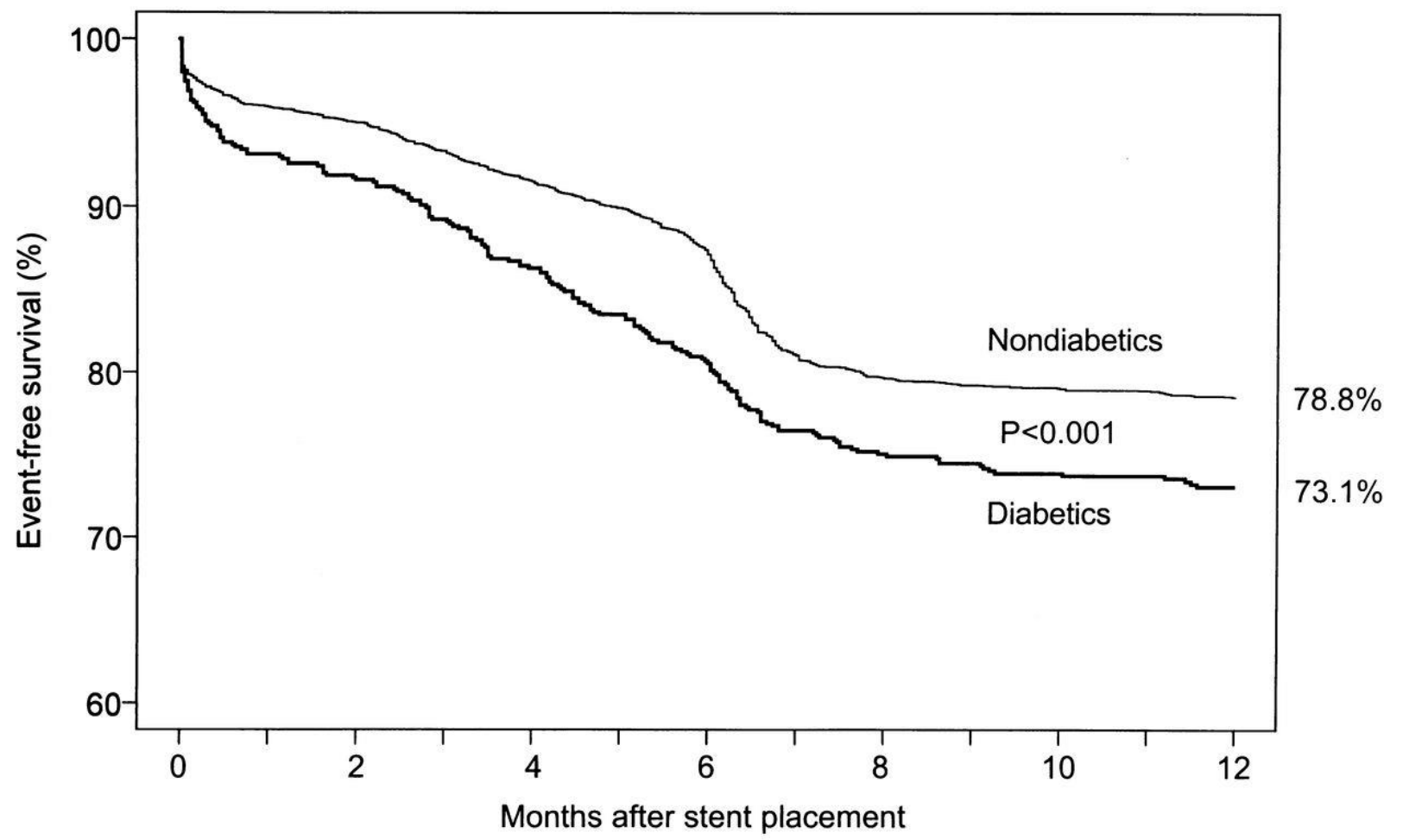

Figure 1.2: Decreased Event-Free Survival in Diabetic Patients with Vascular Stents [13].

Adverse events include death, cardiopulmonary events (stroke, myocardial infarction, pulmonary embolism) and restenosis.

Every improvement to current practice has closed the gap between diabetic and nondiabetic populations [19], but it persists, and has driven medical device companies to design stents and other vascular interventions specifically for diabetic patients $[15,16]$.

\subsection{Traditional Device Testing Progression}

To ensure patient safety, newly designed medical devices must be thoroughly tested before clinical trials take place. Traditionally, testing applicability of newly designed devices for diabetic patients requires development of diabetic animal models. A perfect animal model for atherosclerosis and vascular stenting in Type 2 Diabetes is elusive. The many attempts to develop such a model attest to this challenge. Canine models were used historically, with stents deployed in coronary and peripheral arteries. However, canine arteries have noticeably different 
coagulation and intimal healing patterns than human counterparts, and results from canine trials failed to predict success of human trials [22].

Artificially-induced atherosclerosis often produces endothelial abrasions on otherwise healthy rats, rabbits, and swine [23], leading to a very different habitat for recovery than a diabetic blood vessel. Drug-induced diabetic animal models and 2D cell-culture models are used in tandem to evaluate stent quality [15], but cannot predict the outcome of clinical testing [1720]. Tissue engineering, a broad term for combining engineered materials and living cells to create functional constructed tissues, has enabled device designers to conduct intermediate research between established 2D benchtop testing methods and costly animal trials [26].

\subsection{Blood Vessel Mimics}

In order to test vascular devices, Cal Poly's Tissue Engineering Lab cultivates tissueengineered blood vessel mimics (BVMs) using polymer scaffolds and human vascular cells. Cells are derived from umbilical cord veins isolated and pooled over multiple donated umbilical cords. Cells from the innermost layer are referred to as Human Umbilical Vein Endothelial Cells (HUVECs). HUVECs are cultured before being harvested and injected into a polymer scaffold.

Scaffolds are fabricated by electrospinning, wherein thin fibers of poly-lactic-co-glycolicacid (PLGA) are pulled by electrostatic attraction to form a thin tube around a translating and rotating mandrel. The scaffold is conditioned in high-protein media to promote cell adhesion before cells are sodded under high pressure to promote penetration into the walls of the scaffold. The scaffold takes the role of the tunica adventitia, the outermost fibrous layer of a blood vessel. In some cases, human smooth muscle cells isolated from the umbilical artery (HUASMCs) are sodded first, creating a muscular tunica media, before human endothelial cells isolated from the umbilical vein (HUVECs) are sodded to create a tunica intima [27]. Figure 1.3 compares blood 
vessel layers to corresponding components of the BVM. To isolate the endothelial dysfunction response to elevated glucose, models in this thesis contain only HUVECs. Over time, the cells form a robust layer, which can respond to environmental conditions like high glucose concentration or foreign objects like implanted stents.
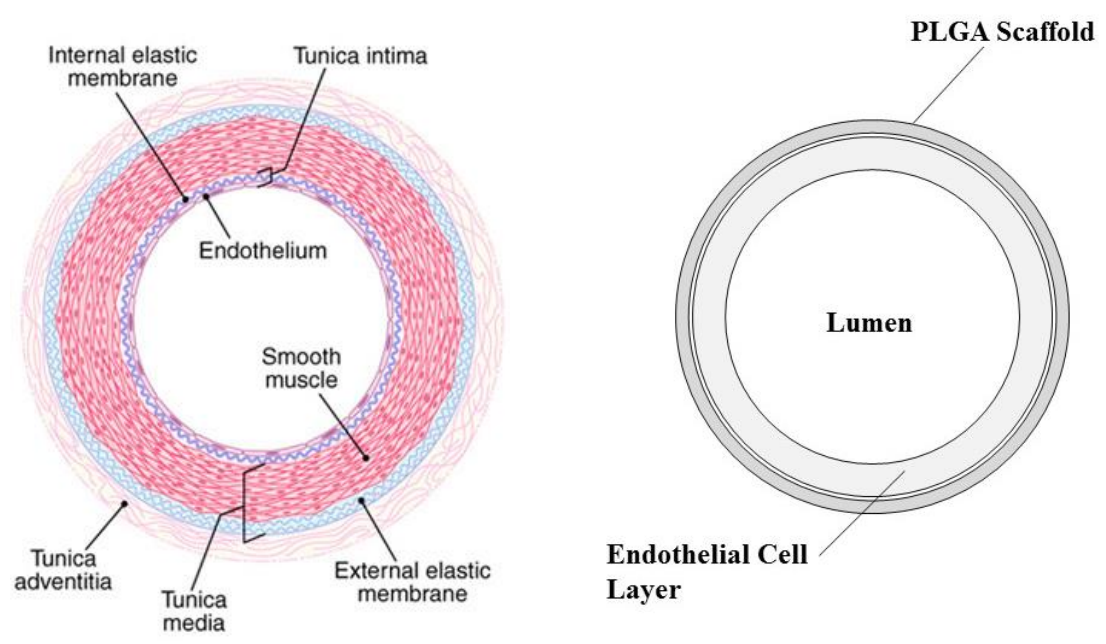

Figure 1.3: Blood Vessel and BVM Layers. Blood vessels are composed of tunicae intima, media, and adventitia, while BVMs are composed of polymer scaffolds and cell linings.

Scaffolds are incorporated into a bioreactor, a closed system whereby cell media can be pumped through vessel mimics under pulsatile flow. Figure 1.4 depicts the path of cell media through the peristaltic pump, bioreactor, and cell media reservoir. 


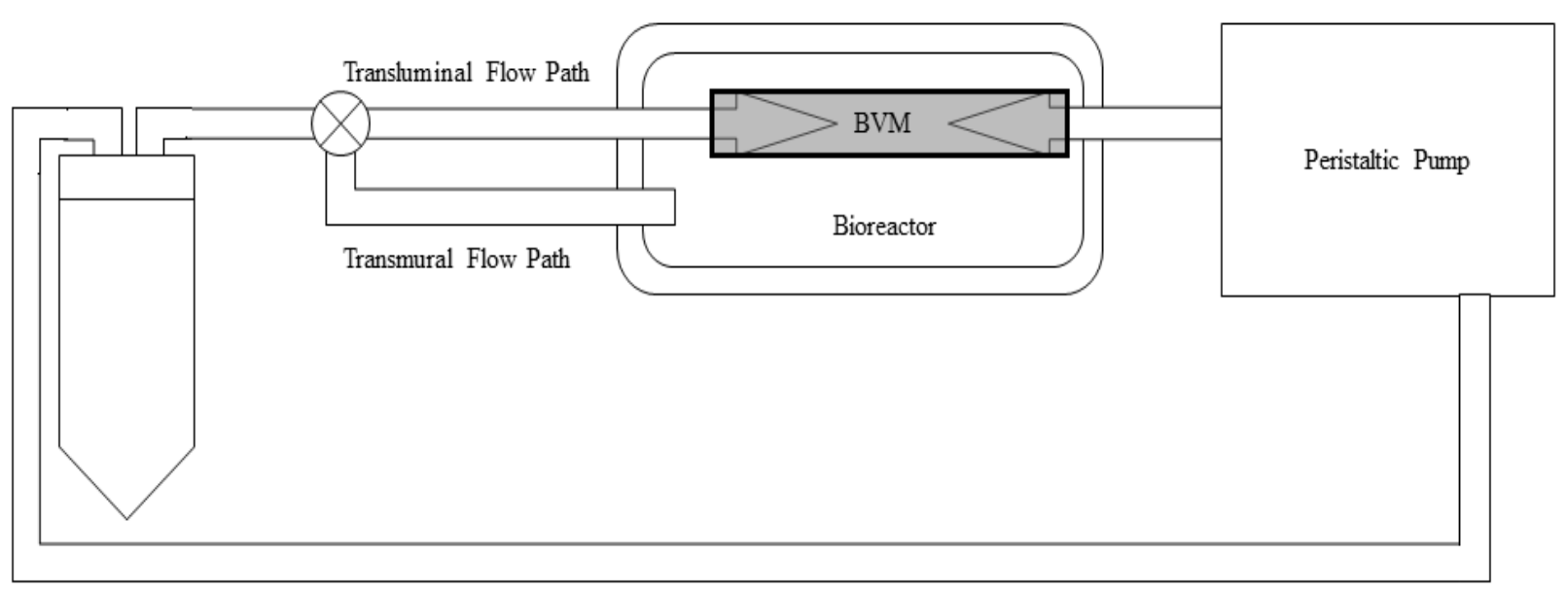

Figure 1.4: Bioreactor Schematic in the Cal Poly Tissue Engineering lab. Cells are sodded into polymer scaffold before incorporation into peristaltic flow system. Cell media is pushed through tubing, through the lumen of the BVM, circulating as cell layer develops. Stopcocks isolate flow through transmural or transluminal paths.

\subsubsection{Inducing Elevated Glucose Conditions in BVMs}

To replicate the hyperglycemic conditions present during prediabetes and Type 2

Diabetes, additional glucose can be added to cell media [28]. Standard cell media contains 5.5 $\mathrm{mM}$ glucose as a source of cellular energy, meant to reproduce the concentration of glucose in the blood in a normoglycemic individual. To imitate prediabetic conditions, glucose concentration can be increased to $10.5 \mathrm{mM}$; to imitate diabetic conditions, the concentration can be $25.5 \mathrm{mM}$ (Table 1.1). Moderate glucose media was made by combining $8.40 \mathrm{~mL} 80 \mathrm{mM}$ sterile glucose solution with $116.6 \mathrm{~mL}$ cell media, and high glucose media was made by combining $33.55 \mathrm{~mL} 80 \mathrm{mM}$ glucose with $91.45 \mathrm{~mL}$ cell media. These concentrations have been previously established as sufficient to influence cell behavior as measured by changes in gene expression [28]. The glucose concentration required to detect changes in viability representative of diabetic conditions is higher in cultured cells compared to recently-isolated primary cells. This difference is related to changes in glucose utilization of proliferating cells in culture $[29,30]$. 
Table 1.1: Elevated Glucose Conditions

\begin{tabular}{|c|c|c|c|}
\hline Treatment & Total Glucose & Total Glucose & Represented Disease State [22] \\
\hline Label & $\begin{array}{l}\text { Concentration } \\
(\mathrm{mM})\end{array}$ & $\begin{array}{l}\text { Concentration } \\
(\mathbf{m g} / \mathbf{d L})\end{array}$ & $\begin{array}{l}\text { (Diagnostic Fasting Plasma } \\
\text { Glucose) }\end{array}$ \\
\hline Basal & 5.5 & 99 & $\begin{array}{l}\text { Normoglycemic } \\
(<100 \mathrm{mg} / \mathrm{dL})\end{array}$ \\
\hline Moderate & 10.5 & 189 & $\begin{array}{l}\text { Prediabetic } \\
(100-125 \mathrm{mg} / \mathrm{dL})\end{array}$ \\
\hline High & 25.5 & 459 & $\begin{array}{l}\text { Diabetic } \\
(>126 \mathrm{mg} / \mathrm{dL})\end{array}$ \\
\hline
\end{tabular}

Exposing BVMs to high glucose conditions is the first step toward potentially approximating the environment of a diabetic blood vessel. If the conditions are sufficiently similar, the BVMs should exhibit oxidative stress, a well-documented cellular response to elevated glucose $[9,24,25]$.

\subsection{Cellular Responses and Expression of mRNA and Proteins}

\subsubsection{Cellular Response to Oxidative Stress and qPCR}

Glucose molecules signal mitochondria to increase production of ROS, which bind and inactivate a wide range of molecules within the cell. This process in turn further increases ROS production through a positive autoregulatory feedback loop. An imbalance of ROS and ROSquenching molecules, or antioxidants, leads to a condition known as oxidative stress. Oxidative stress can be useful to the body, signaling immune responses to foreign antigens. In chronic oxidative stress conditions, however, it leads to endothelial dysfunction, the precursor to 
macrovascular damage characteristic of diabetes. This occurs when stress-sensitive pathways are activated, notably the nuclear factor kappa-light-chain-enhancer of activated B cells (NF- $\mathrm{B})$ pathway. ROS and inflammatory cytokine interleukin-6 (IL-6) bind their extracellular receptors, initiating a signaling cascade which activates $\mathrm{NF}-\kappa \mathrm{B}$. When activated, NF- $\kappa \mathrm{B}$ translocates into the nucleus and acts as a transcription factor for inflammatory cytokines like IL-6 and CD36 and sorbitol, which in turn increases ROS generation and produces AGEs. Figure 3 depicts NF- $\mathrm{B}$ and connected pathways, including mitogen-activated protein kinase (MAPK) and Janus kinase/signal transducers and activator of transcription (JAK/STAT) pathways. These increase insulin resistance and Beta cell dysfunction, the systemic metabolic implications of diabetes. They also trigger endothelial dysfunction in the forms of chronic vasoconstriction, arterial stiffening, and development of atherosclerosis [33].

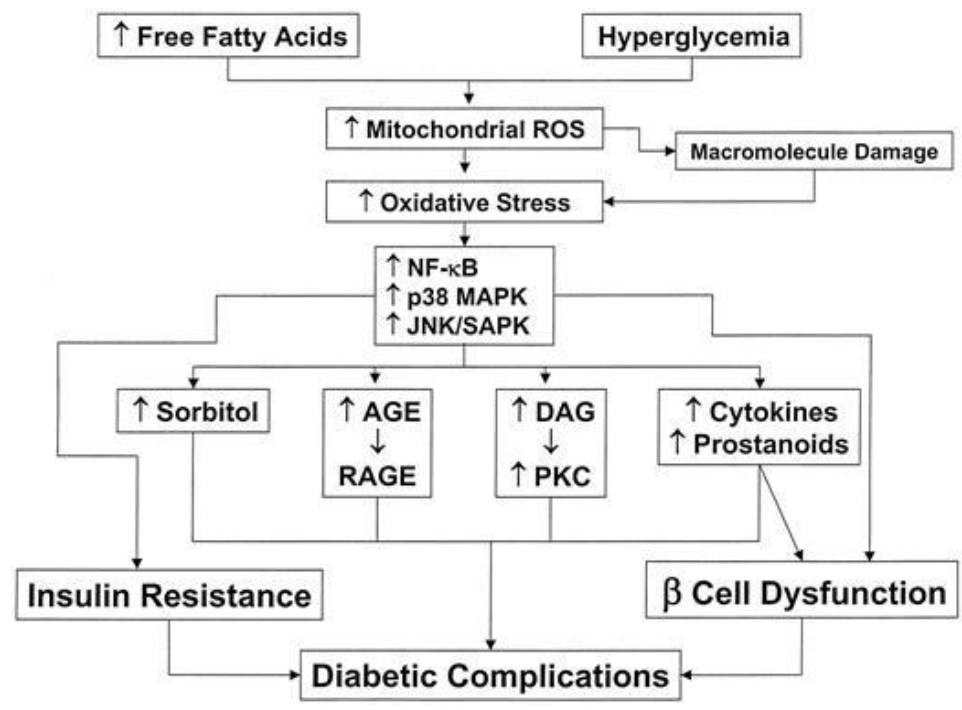

Figure 1.5: Pathways Involved in Hyperglycemia-Induced Endothelial Damage. Many pathways interact to initiate diabetic macrovascular disease. Both targets for gene expression analysis are mechanistically understood to be upregulated under hyperglycemic conditions [8]. Interleukin 6 (IL-6) is both an inflammatory chemokine which initiates the appropriately named "signal transducer and activator of transcription" (JNK/STAT) pathway and a product of 
the same pathway. This gives IL-6 both autocrine feedback to the same cell, upregulating soluble IL-6 production and further increasing mRNA abundance therein [34], and paracrine feedback to nearby cells, which creates signal amplification in localized tissues. Increased IL-6 activity with increased extracellular glucose concentration therefore could represent one step toward a successful model of diabetic macrovascular disease.

Cluster of Differentiation 36 (CD36) is a cell surface scavenger receptor and has been shown to increase production of ROS and smooth muscle cell endocytosis of oxidized LDL, a building block of atherosclerotic plaques [35]. Mechanistically, CD36 binds its receptor and triggers the PPAR- $\gamma$ pathway [36]. This pathway boosts production of CD36 and fatty streak accumulation in vivo. Increasing CD36 expression correlated with elevated glucose concentration is therefore another potential accomplishment in designing a BVM with diabetic vascular disease [31]. After identifying the key pathways promoted by a high glucose environment, determining a means of measurement would be necessary to reveal how closely BVMs in these conditions replicate human blood vessels undergoing disease processes.

\subsubsection{Reverse-Transcription Quantitative Polymerase Chain Reaction (RT-qPCR)}

Cellular response to high-glucose conditions can be assessed using reverse-transcription quantitative polymerase chain reaction (RT-qPCR). Polymerase chain reactions (PCR) amplify the amount of a target sequence of DNA which can be used for further study. PCR utilizes the enzyme DNA Polymerase, which covalently bonds corresponding Watson-Crick base pairs with the template DNA strand. DNA templates here are from the treated cells, and additional free nucleotides are added to the solution. The solution is heated, or denatured, to break hydrogen bonds between the two strands of DNA, exposing unpaired nucleotides to be copied. DNA Polymerase cannot bind to the template strand without a complementary nucleotide primer in 
place on the template strand, which creates a small double-stranded section. This allows for extremely targeted replication of a specific DNA fragment. Under engineered conditions, DNA Polymerase replicates the DNA exponentially over repeated cycles of denaturation, annealing, and extension.

Quantitative PCR (qPCR) uses the same enzymes and principles as traditional PCR but adds a fluorescent reporter molecule, which emits light proportionally to the number of doublestranded DNA molecules present during any given cycle of PCR. qPCR instruments analyze and report fluorescence during each successive cycle, and due to the programmable temporal scale of PCR, allow for back-calculation of starting copy number for each sample. Exponential amplification of target sequences allows for differentiation between relatively similar starting copy numbers, offering accurate measurements of relative abundance of specific gene sequences between samples.

Reverse transcription (RT) is an enzymatic reaction which uses Reverse Transcriptase to transcribe complementary DNA (cDNA) from RNA. Within a cell, RNA transmits communication between the nucleus and the organelles responsible for modifying cell behavior. Messenger RNA (mRNA) in particular relays information by acting as the template in the process of encoding proteins. When extracellular signals initiate cascades that evolved in response to stress, transcription factors bind nuclear DNA in specific places, increasing the number of copies of those particular mRNAs in order to increase production of the associated protein. This process is how cells respond to their environments, making mRNA abundance an appropriate indicator of the activation of stress-response pathways. 


\subsubsection{Genes of Interest}

Due to the granularity of the differences in relative mRNA abundance elucidated by RTqPCR across treatment groups, genes must be selected to accurately represent changes in cell behavior. The genes CD36 and IL-6, the functions of which are described in detail above, were chosen for study through RT-qPCR in the hopes of capturing the acute endothelial cell response to increased extracellular glucose.

\subsubsection{Reference Genes}

To accurately compare levels of mRNA expression across various samples, concentrations of nucleic acid must be identical. This is approximated as closely as possible by measuring RNA concentration before reverse transcription to cDNA, but to further control for variability, samples are also assessed for mRNA abundance of genes which should not be affected by increasing extracellular glucose. Three reference genes are evaluated in this thesis. Beta-actin $(A C T B)$

ACTB encodes for one of two cytoskeletal actin proteins [37]. All human cells express ACTB similarly and cytoskeletal function is not known to be affected by extracellular glucose, making ACTB a suitable reference gene with which to normalize qPCR results.

\section{Beta-2 microglobulin (B2M)}

B2M encodes for a component of the Major Histocompatibility Complex 1 (MHC Class 1), which allows cells to display non-self-proteins to cytotoxic T cells [38]. This is an important function of immunity, and MHC class 1 is found on all nucleated human cells (excluding red blood cells). 
Hypoxanthine-guanine phosphoribosyltransferase (HPRT1)

HPRT1 encodes for protein HGPRT which is a catalytic enzyme involved in synthesizing purine nucleotides, adenine and guanine [39]. Nucleotides are required for translation and transcription of genetic material, making HPRT1 essential to all human cells.

\subsubsection{Reactive Oxygen Species and Fluorescent Microscopy}

To complement qPCR-based gene expression analysis, fluorescent staining for cell viability and ROS expression can be analyzed across glucose treatment groups. Cell viability gives a general estimation of how well cells are surviving and replicating [40]. Extracellular glucose interferes with cell survival by upregulating mitochondrial production of ROS, signaling apoptosis via the transcription factor Sp1 [27, 28]. Cell death due to hyperglycemia is a major component of endothelial dysfunction and the pathology of diabetic macrovascular disease [43] so visualizing and quantifying cell viability offers insight into progression of high glucoseinduced oxidative stress pathways. Decreasing cell viability by approximately $12 \%$ with increasing glucose concentrations contributes to a physiologically relevant model [27, 30].

\subsection{Summary and Aims of this Thesis}

In progression toward a tissue engineered blood vessel mimic system suitable for testing devices intended to treat diabetic macrovascular disease, this thesis pursued the following specific aims:

Aim 1: To identify genetic corollaries that indicate increased oxidative stress responses in endothelial cells, and to determine a method by which to study specific corollaries in gene expression.

Evaluation of oxidative stress response pathway activity by RT-qPCR requires selection of genes whose change in regulation will represent pathway activation. Chapter 2 details this 
selection process. Data analysis methods for RT-qPCR make use of reference genes that remain constant across treatment groups. Determination of the suitability of those reference genes as well as comparisons between mRNA expression of genes of interest depends heavily on the methodology used for data analysis. Principles of and popular approaches to data analysis are also discussed in Chapter 2.

Aim 2: To evaluate endothelial cell expression over time for the markers CD36 and IL6 in titered glycemic conditions representative of healthy, prediabetic, and diabetic individuals.

Based on data analysis protocols and genes of interest determined in Chapter 2, an examination of gene expression in Human Umbilical Vein Endothelial Cells (HUVECs) treated with extracellular glucose concentrations representative of diabetic progression over seven and fourteen-day timepoints was conducted. Results of these experiments are presented and discussed in Chapter 3.

Aim 3: To assess effect of elevated glucose over time on in vitro endothelial cell viability and reactive oxygen species (ROS) production at titered glucose concentrations representative of healthy, prediabetic, and diabetic conditions.

Cell viability and ROS product were measured using a combination of fluorescent stains and confocal microscopy techniques. Fluorescent staining permits image-based analysis of cell viability and function by differentiating live and dead cells and by fluorescing proportionally to the amount of cell products generated. Imaging-based analysis is particularly relevant to the BVM system because these stains can be used to evaluate blood vessel mimics as a whole. Staining, imaging, and image processing are described in detail in Chapter 4. 


\section{DETERMINING GENES OF INTEREST AND OPTIMIZING QPCR DATA}

\section{ANALYSIS}

\section{1: Introduction}

Tissue engineered models are inherently imperfect; it is impossible to capture a complex disease process in a model disconnected from a living organism. Measuring this inaccuracy proves to be a difficult task in itself, as there are dozens of cell types with hundreds of genes with known involvement in development of atherosclerosis and diabetic macrovascular disease [45]. Gene expression analysis is a powerful tool for evaluating cellular responses, granted that the genes analyzed accurately represent those responses, and that data is analyzed properly. Gene selection involves reviewing literature to identify prominent pathways and choosing targets that have established response patterns in vivo in diabetic patients. Data analysis depends on thorough experimental controls and appropriate comparisons, as spurious conclusions can emerge when data is mishandled. This chapter describes preliminary decisions and experiments necessary to compare the elevated glucose BVM model to the diabetic macrovascular disease state.

\section{2: Gene Selection and Primer Acquisition}

A process as complex as the development of diabetic macrovascular disease involves dozens of individual genes coordinating responses to varying stimuli [35]. Known oxidative stress pathways involve multiple points of origin and dozens of potential genetic targets of analysis.

Genes investigated to represent diabetic macrovascular disease were CD36, CD68, COX2, iNOS, IL-1A, IL-6, PLA2G7, and PPAR $\gamma$. Proinflammatory cytokines CD36, CD68, IL1A, and IL-6 are upregulated in Type 2 Diabetes and initiate a positive feedback loop by which acute inflammation becomes uncontrolled [24, 35-37]. Uninhibited inflammation contributes to 
endothelial dysfunction present in diabetic macrovascular disease [7]. PLA2G7 or phospholipase A2 converts circulating phospholipids to arachidonic acid which is cleaved by COX2 or cyclooxygenase-2 into prostaglandin $\mathrm{H} 2$ [38-40]. The presence of COX2 rather than COX1, a functionally similar molecule, is correlated with hyperglycemia-induced inflammation [50]. Inducible nitric oxide synthase (iNOS) gene variants are associated with poorer clinical outcomes in Type 2 diabetic patients [52] and animal models correlate increased iNOS expression with endothelial dysfunction [53]. Finally, PPAR $\gamma$ is a master regulator of fatty acid uptake and is implicated in development of atherosclerosis [54]. Genes that were considered as experimental targets for this thesis are summarized in Table 2.1.

Table 2.1: Initial Genes of Interest

\begin{tabular}{|l|l|l|l|}
\hline Gene $^{\dagger}$ & Function & Pathway & Ref. \\
\hline CD36 & Binds oxLDL and AGE-modified proteins & PPAR $\gamma$ & {$[31]$} \\
\hline CD68 & Correlated with insulin sensitivity and IL-6 secretion & NFאB & {$[46]$} \\
\hline COX2 & Proinflammatory, associated with Type 2 Diabetes & AA & {$[50]$} \\
\hline iNOS & Oxidative stress, NO production & MAPK & {$[53]$} \\
\hline IL-1A & Inflammatory cytokine & NFאB, MAPK & {$[47]$} \\
\hline IL-6 & Immunoregulation, glucose metabolism & JNK/STAT & {$[48]$} \\
\hline PLA2G7 & Promotes oxLDL formation, plaque instability & AA/MAPK & {$[49]$} \\
\hline PPAR $\gamma$ & Glucose metabolism, fatty acid uptake, master regulator & PPAR $\gamma$ & {$[55]$} \\
\hline
\end{tabular}

$†$ HGNC official gene names $\$$ Arachidonic acid pathway

Once these 8 primary genes of interest were identified, the next step was to acquire PCR reagents to evaluate their utility. This took place in three steps: initial primer design, preliminary verification of custom primers, and a decision to use preverified primers. Primer sequences for 
all 8 genes and purchasing information for all qPCR analyses are provided in Appendix A and will be described below.

\subsection{1: Initial Primer Design}

Multiple primer sequences were designed and tested for each of the eight preliminary gene targets. Sequences were acquired from the NCBI gene sequence database GenBank and primers were designed using the IDT PrimerQuest tool (idtdna.com/primerquest) and the NCBI Primer BLAST tool (https://www.ncbi.nlm.nih.gov/tools/primer-blast).

To summarize the process, sequences were searched for in the GenBank Homo sapiens mRNA database by HGNC name. Sequences were then entered into PrimerQuest and custom design parameters were chosen. Primer size was limited to 18-22 nucleotides to encourage binding only onto genes of interest. Primer melting temperature, Tm, was limited to $59-61{ }^{\circ} \mathrm{C}$ to facilitate evaluation of multiple primer sets using the same qPCR settings. Amplicon size was limited to $180-220 \mathrm{bp}$ to equalize annealing times across PCR products and also facilitate evaluation of multiple genes using the same qPCR settings. PrimerQuest then generated five ranked potential primer sets which were copied to Primer BLAST to check for homology with non-target genes. Primer BLAST tested inputted sequences against all consensus mRNA sequences and primers were deemed acceptable when only target genes were returned. This step ensured only the gene of interest was amplified. Once the primers were confirmed to have no homologs, the highest-ranked primer sets were selected and ordered from the PrimerQuest tool. A detailed protocol for primer design, ordering, and selection can be found in Appendix B.

Figure 2.2 shows Delta Rn vs Cycle Number for one or two custom primer sets for each of the eight preliminary genes of interest, totaling 14 sets. Delta $\mathrm{Rn}$ is the derivative of fluorescence intensity and Cycle Number represents qPCR progression. Many of these primers 
failed to amplify, demonstrated by lines that never crossed the horizontal threshold line through the linear amplification region ("B"). Some reactions, including one shown in light green in Figure 2.1 ("A"), had irregular amplification patterns that suggest the formation of unwanted PCR products.

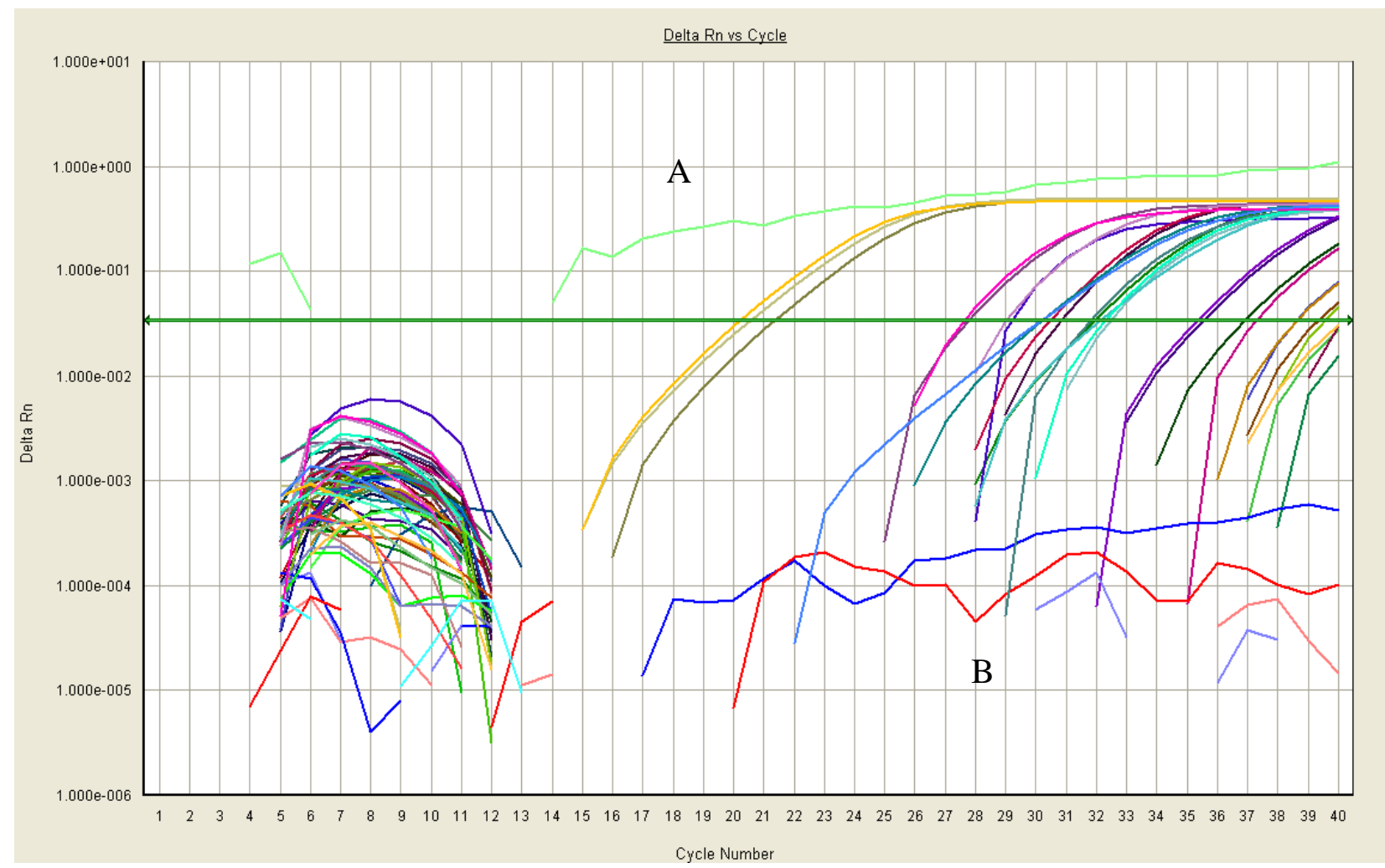

Figure 2.1: Amplification Plot of All Initial Primers. Each line represents one individual qPCR sample. The $x$-axis represents cycle number, or progress of the qPCR, while Delta Rn on the $y$ axis represents change in fluorescence intensity. The Delta Rn value is the derivative of fluorescence intensity. Sample A shows irregular replication while Samples in Region B failed to replicate.

Irregular replication and lack of replication in many wells demonstrated the faults of these primers. Many amplification curves intersected, implying unequal reaction efficiency across wells. 


\subsubsection{Methods: qPCR and Visual Analysis for Primer Quality}

As described briefly in Chapter 1, qPCR measures relative expression of genes across samples by repeatedly separating and replicating target strands. Primers obtained for each gene (Appendix A), template cDNA, DNA polymerase, unpaired nucleotides (dNTPs), and enhancers were combined for each reaction into well plates. Well plates were placed in a thermocycler with qPCR functionality. To begin qPCR, samples were heated to $95^{\circ} \mathrm{C}$ to denature, or separate, strands of DNA. Samples were then cooled to $60^{\circ} \mathrm{C}$ to allow primers to bind to their complementary sequences, at which point DNA polymerase extended to replicate the template strand. Intercalating dye molecules fit between base pairs of DNA as strands anneal and extend, with the amount of intercalated dye being proportional to the amount of DNA present. The thermocycler then measured fluorescence of intercalating dye, marking the completion of one cycle of the reaction. Following completion of forty cycles, a dissociation step tracked fluorescence as temperature gradually increased. Temperature at which PCR products dissociate is determined by identity, so all products which dissociated at approximately the same temperature were assumed to be equivalent. A complete protocol for preparing and running qPCR is available in Appendix C.

During qPCR, measured fluorescence in each well was reported by the thermocycler during each reaction cycle. Amplification curves were generated from these measurements and facilitate comparison of reactions. As cycles progressed, fluorescence increased linearly before reaching a plateau as available nucleotides were depleted. In order to compare relative abundance, a common threshold was established for all samples by drawing a line across the linear region of all amplification curves on the plate. The cycle at which each sample reached the 
threshold is known as the $\mathrm{C}_{\mathrm{T}}$, or "cycles to threshold," value. The $\mathrm{C}_{\mathrm{T}}$ value of each sample is the basis for comparing relative abundance. Figure 2.2 depicts an amplification plot and labels its linear region, threshold, and $\mathrm{C}_{\mathrm{T}}$ value.

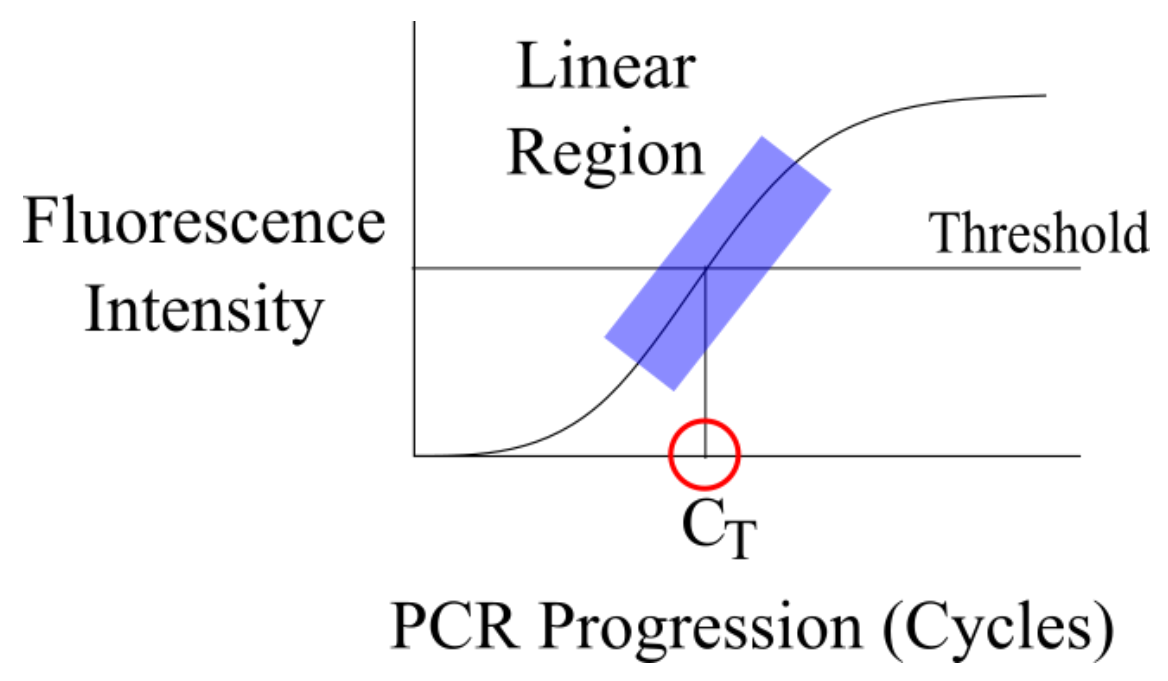

Figure 2.2: Amplification Plot with Linear Amplification Region, Threshold, and $C_{T}$.

Primers were tested by RT-qPCR with cDNA derived from Human Umbilical Vein Endothelial Cells (HUVECs) (Lonza, C2519A) at passage 5 cultured in corresponding cell culture media (Lonza, EGM Plus) to $80 \%$ confluence in T-75's and passaged at 1:3 ratios throughout culture. RNA isolation and cDNA synthesis were performed before qPCR and generation of corresponding amplification plots. Amplification curves for each set of primers were evaluated visually for similarity of amplification across replicates. Amplification curves which intersected in the linear region demonstrated unequal reaction efficiencies and were rejected. Amplification curves which lacked the expected biconcave shape were also rejected, including those which never crossed the threshold line. 


\subsubsection{Preliminary Primer Verification}

During primer selection, primers that failed visual evaluation were rejected. The five sets of custom primers that successfully replicated are presented in Figure 2.3 as a demonstration of primer selection. Genes represented are CD68, IL-1A, PPAR $\gamma$, and PTGS2 (two successful sets). CD36 and IL-6 primer sets failed to replicate.

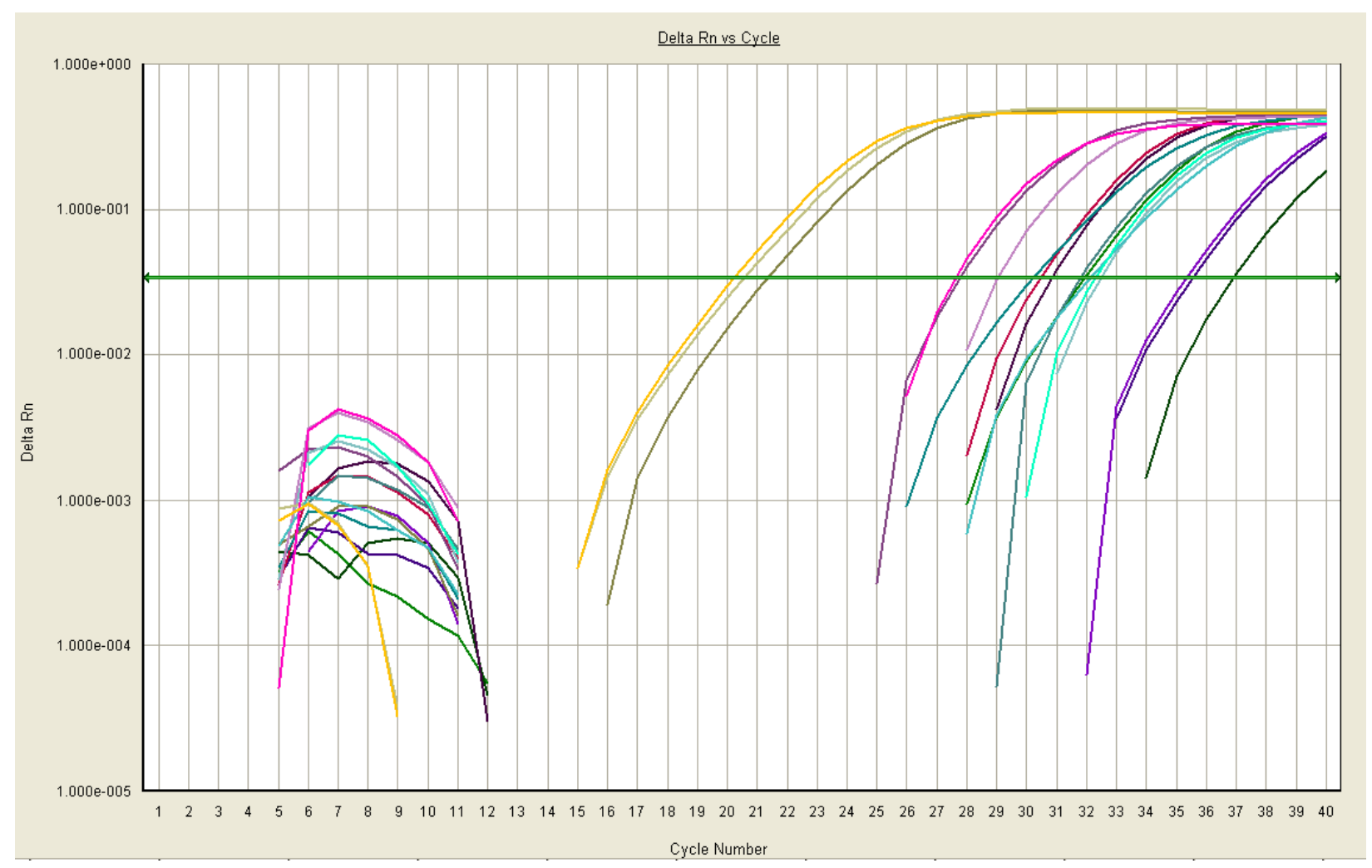

Figure 2.3: Amplification Plots of Successful Initial Primers. Isolated here are only those primers which produced three CT values approximately equal to one another. As in Figure 2.2,

the $x$-axis represents $P C R$ progression and the $y$-axis represents change in fluorescence intensity. Each line represents one sample qPCR well.

This was only the first validation step required to reliably make conclusions from custom primers, as variability of replication efficiency is evident in Figure 2.3 where amplification curves intersect. A complete protocol for primer selection is available in Appendix B. 


\subsubsection{Preverified Primers}

Although the primers in Figure 2.3 were appropriate for further validation, they were not ultimately used in this thesis. Failure to replicate CD36 and IL-6 genes was especially problematic as these genes are particularly well-studied compared to other genes of interest. Owing to the recurring associations of CD36 and Il-6 with diabetes and endothelial dysfunction, these two gene targets were necessary to consider given the aims of this thesis. CD36 mRNA expression was previously documented to increase in response to elevated glucose concentrations in microvascular endothelial cells and this upregulation was demonstrated to mediate oxidative stress [31]. In a genome-wide study, CD36 variants were associated with changes in lipoprotein diameter in response to fenofibrate, an insulin resistance treatment, indicating CD36 may be related to insulin resistance through lipoprotein pathways associated with atherosclerosis [56]. A CD36 knockout mouse model (mice without the gene for CD36) demonstrated decreased inflammation and increased insulin signaling compared to wild-type mice [57]. Plasma IL-6 concentration and advanced glycation end products were both shown to have increased in diabetic patients compared to non-diabetics [58] and IL-6 was recently shown to be involved with procoagulant molecule thrombin in the transition from acute to chronic inflammation [34]. Acquiring primers to evaluate expression of CD36 and IL-6 required repetition of custom primer design or pursuit of another solution.

Due to the cost of repeating attempts at primer design and verification, and in an effort to streamline data acquisition, pre-verified primers were purchased for CD36 and IL-6. The increased cost of these primers (approximately $\$ 130$ for one preverified set compared to $\$ 15$ for one set of custom primers) necessitated a reduction in the number of experimental targets, so other genes of interest were not investigated further in this thesis. PrimeTime qPCR primers 
(IDT DNA, Coralville, IA) arrived having been tested to ensure that the provided set of forward and reverse primers bound complementary DNA (cDNA) at the location of the gene of interest and reliably produced replicable qPCR results. This eliminated the need for primer verification and reduced the amount of cDNA derived from valuable human cells necessary to test primers. Sequences of PrimeTime primers (for CD36, IL-6, and reference genes described below) used for all subsequent experiments are available in Appendix A.

Predesigned primers were also used to measure relative expression of reference genes. This decision was made due to the use of preverified primers for genes of interest, as well as to facilitate comparison with these experiments in future research conducted on the diabetic macrovascular disease BVM model. Three genes, whose functions were previously discussed in Chapter 1, were considered as reference genes for data analysis. The purpose of reference genes is to normalize activity of genes of interest to a gene that maintains consistent expression across treatment groups. The three reference genes analyzed by qPCR were B2M, ACTB, and HPRT1. Though ideal replication behavior was expected from all pre-verified PrimeTime primers, preliminary evaluations were performed using methods described above for custom primers. Amplification for IL-6, CD36, HPRT1, and B2M was consistent across replicates, however ACTB displayed variability. Figure 2.4 displays amplification plots of all preverified primers. Figure 2.4 ("B”) denotes amplification curves for ACTB, which had greater variability than expected. 


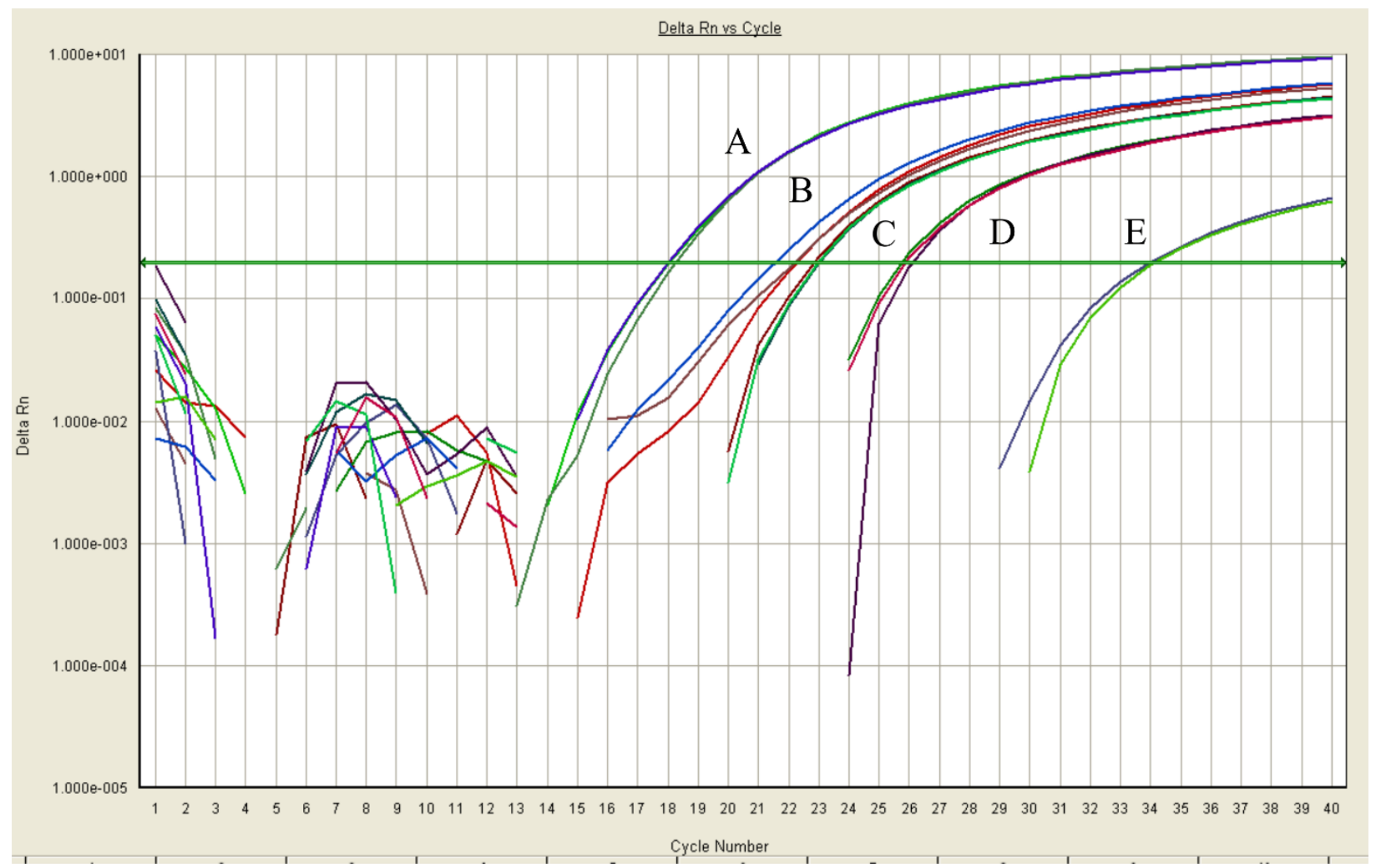

Figure 2.4: Amplification Curves of PrimeTime Preverified Primers (A)IL-6 (B)ACTB (C)B2M

(D)HPRT1 (E)CD36. ACTB (B) displays greater variability among replicates than other sets.

As previously mentioned, a dissociation step occurred following qPCR cycles where temperature was gradually increased, and fluorescence was recorded. When PCR products were identical, they all dissociated at the same temperature, emitting fluorescence. Multiple peaks on a plot of fluorescence intensity against dissociation temperature therefore demonstrated formation of more than one qPCR product. Due to increased variability in ACTB amplification curves, consultation of dissociation curves was warranted. Dissociation curves for ACTB primers had a peak in the non-template control well visible at a much lower temperature than the sample wells. This demonstrated that primers had bound together, referred to as primer-dimer formation.

Figure 2.5 compares dissociation curves from ACTB samples to those from IL-6, which does not display primer-dimer formation. 

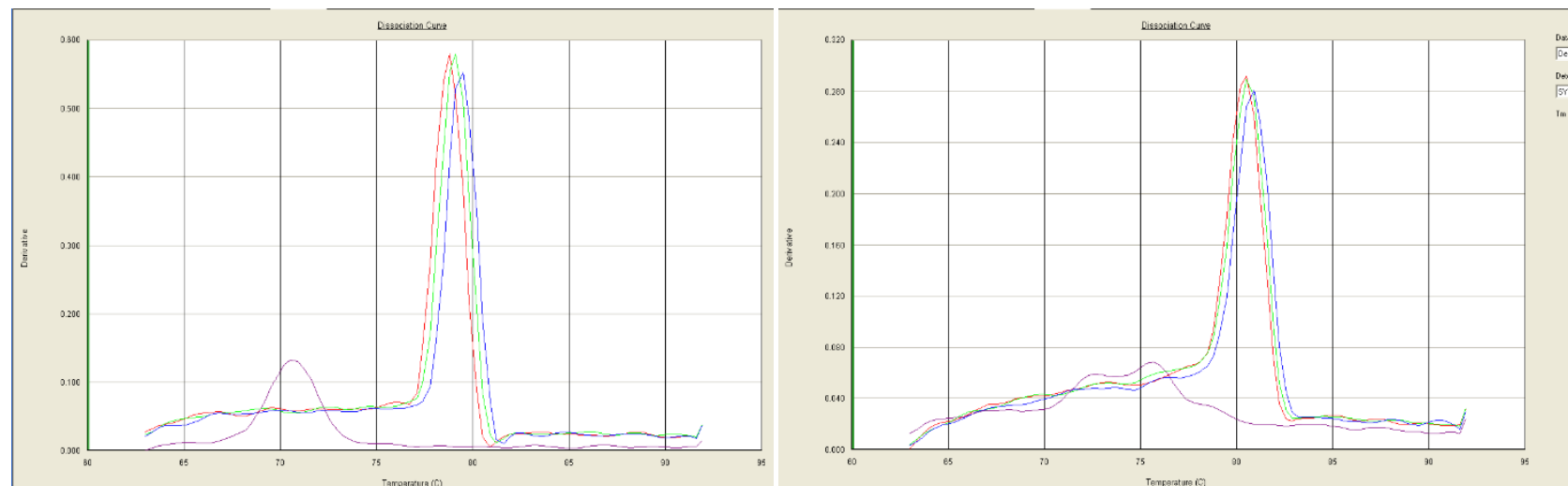

Figure 2.5: Dissociation curves of (A) ACTB and (B) IL-6. ACTB has a peak in the non-template control sample, demonstrating primer-dimer formation.

Eliminating ACTB due to primer-dimer formation determined two target genes, IL-6 and CD36, and two reference genes, B2M and HPRT1. These primers were used in all subsequent analysis throughout this thesis. With these genes established and primers validated, data could be collected. Data from qPCR required processing before interpretation, however, with multiple data analysis methods to choose from. The following section offers comparison of data analysis methods and discusses the selection of the most relevant method for this thesis.

\subsection{Data Analysis Methods}

Differences in mRNA abundance can be compared using two broad approaches: absolute or relative quantification. Both approaches were used in this thesis. Absolute quantification relies on dilution assays to determine a standard curve for each reaction. In this thesis, absolute quantification was used to calculate reaction efficiency $(\mathrm{E})$, which facilitated comparison of starting mRNA abundance $\left(\mathrm{E}^{-\mathrm{C}_{\mathrm{T}}}\right)$ where $\mathrm{C}_{\mathrm{T}}$ is cycles to threshold as determined by $\mathrm{qPCR}$. Relative quantification traditionally relies on the simplifying assumption of perfect efficiency $(E=2.0)$ and three prevalent methods are named for the values compared using this assumption: the $2^{-\mathrm{C}} \mathrm{T}, 2^{-\Delta \mathrm{C}} \mathrm{T}$, and $2^{-\Delta \Delta \mathrm{C}}$ Tethods $[52,53]$. This section details each of these methods and their application to a preliminary dataset. 


\subsubsection{Preliminary Dataset Acquisition}

Throughout this chapter, data from one experiment of the many presented in detail later in this thesis were utilized to compare appropriateness of various data analysis methods. Briefly, these data came from Human Umbilical Vein Endothelial Cells (HUVECs) (Lonza, C2519A) cultured in EGM-Plus media (Lonza, C5035) treated with supplemental glucose (VWR, 97062880) for seven days. Three glucose concentrations were compared: Basal (5.5mM), Moderate (10.5 mM), and High (25.5 mM) treatment groups. RNA and cDNA were isolated and synthesized respectively before qPCR was used to determine a Cycles to Threshold $\left(\mathrm{C}_{\mathrm{T}}\right)$ value for each sample. (These methods are described in more detail in the following chapter.)

\subsubsection{Absolute Quantification}

Absolute and relative quantification approaches were utilized in combination in this thesis in order to compare mRNA expression. Efficiency values (E) were first determined by absolute quantification before relative quantification methods were compared with and without the assumption of perfect efficiency. Serial dilutions of cDNA from Basal treatment groups were used as templates in otherwise identical reactions. $\mathrm{C}_{\mathrm{T}}$ values were plotted against dilution factor and a line of best fit was determined where the slope of this line is equal to $\frac{-1}{\log E}$. $E$ is the efficiency of the qPCR reaction, or how many copies of a gene were made from each template strand during each cycle. A complete protocol for qPCR for absolute quantification and efficiency calculation is available in Appendix C.

\subsubsection{Relative Quantification}

There are three primary comparisons that can be made using relative quantification depending on conditions met. If efficiency is assumed to be optimal, $2^{-\mathrm{C}} \mathrm{T}$ comparisons can demonstrate which mRNA have the highest starting abundance. If a treatment can be compared 
to an untreated condition, $2^{-\Delta \mathrm{C}}$ comparisons are appropriate. The $2^{-\Delta \mathrm{C}} \mathrm{T}$ comparison can also demonstrate how multiple genes relate to a stable group of reference genes. With a stable set of reference genes and an untreated condition, fold change or $2^{-\Delta \Delta C}$ T comparisons are appropriate. These fold change comparisons are most physiologically relevant $[45,46]$. Table 2.2 summarizes relative quantification data analysis methods and the assumptions required by each method. These methods are discussed in more detail in the following sections.

Table 2.2: Relative Quantification Methods

\begin{tabular}{|l|l|}
\hline Method & Assumptions \\
\hline $2^{-C_{T}}$ & $\mathrm{E}=2$ \\
\hline $2^{-\Delta C_{T}}$ (treatment-control) & $\mathrm{E}=2$ \\
\hline $2^{-\Delta C_{T}}$ (target-reference) & $\mathrm{E}=2$, stable reference \\
\hline $2^{-\Delta \Delta \mathrm{C}_{\mathrm{T}}}$ & $\mathrm{E}=2$, stable reference \\
\hline
\end{tabular}

2.3.3.1 The $2^{-C}$ Method

The most direct comparison of $\mathrm{C}_{\mathrm{T}}$ value is facilitated by $2^{-\mathrm{C}_{\mathrm{T}}}$ assessment. This method assumes ideal PCR efficiency — that during every cycle, two copies of each template strand are made and represent treatment groups as individual data points rather than comparisons. This is less likely to show spurious trends in small sample sizes than comparative methods [61]. $2^{-\mathrm{C}} \mathrm{T}$ comparisons are especially relevant to determination of reference gene expression across treatment groups [60]. If $2^{-\mathrm{C}} \mathrm{T}$ values differ significantly, reference genes may be unsuited for use as reference genes in later analysis. $2^{-\mathrm{C}}$ evaluation is the most direct method of assessing relative mRNA abundance.

\subsubsection{The $2^{-\Delta C}$ Method}

To evaluate the change from a negative control or Basal condition to a treated (Moderate or High) condition, the $2^{-\Delta C_{T}}$ value was compared where 


$$
\Delta C_{T}=C_{T}(\text { treatment })-C_{T}(\text { basal })
$$

Comparison between expression of the same gene across a treated and untreated group with the same starting cDNA amount as determined by UV spectroscopy is best represented by the $2^{-\mathrm{AC}} \mathrm{T}$ value [61]. This evaluation was used to determine influence of a treatment on gene expression.

\subsubsection{The $2^{-\Delta \Delta C}$ Method}

If consistent reference gene expression had been validated, comparison of $2^{-\Delta \Delta C}{ }_{T}$ values would describe fold change and facilitate expression comparisons across genes (e.g. "Gene $\mathrm{X}$ is upregulated 4-fold while Gene $\mathrm{Y}$ is only upregulated 2-fold"). $2^{-\Delta \Delta C}$ T value was calculated from expression of a target gene (GOI) and the expression of a reference gene [62] (REF) using the following equation:

$$
\begin{gathered}
\Delta \Delta C_{T}=\left\{\left(C_{T} \text { (treatment }, G O I\right)-C_{T}(\text { treatment }, R E F)\right)-\left(C_{T} \text { (basal, GOI }\right) \\
\left.\left.-C_{T}(\text { basal, } R E F)\right)\right\}
\end{gathered}
$$

Complete protocols for all relative quantification methods are available in Appendix D.

\subsection{Data Analysis Results}

\subsubsection{Absolute Quantification}

Absolute quantification was used in this thesis to evaluate the assumption of perfect efficiency traditionally required to perform relative quantification. Determination of reaction efficiency (E) for each primer set began by performing qPCR on serially diluted cDNA, resulting in delayed PCR progression and increased $\mathrm{C}_{\mathrm{T}}$ values for diluted samples. Figure 2.6 depicts absolute quantification amplification plots of IL-6, CD36, B2M, and HPRT1. 

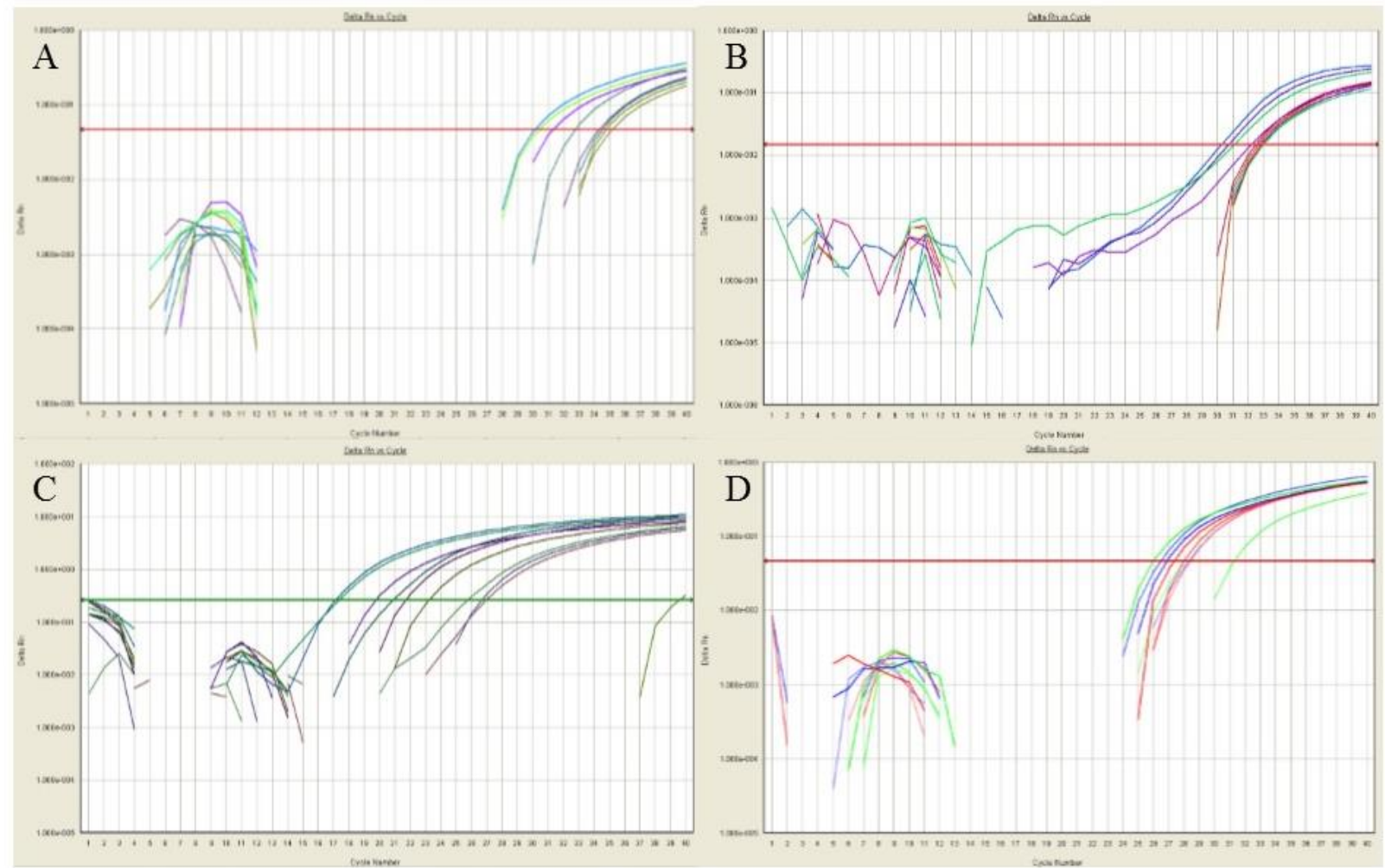

Figure 2.6: Standard Curve Amplification Plots: Change in fluorescence intensity over time (Delta Rn) versus progression of qPCR (Cycle Number) for (A) IL-6 (B) CD36 (C) B2M and (D) HPRT1 pre-verified primers. Horizontal lines depict threshold values.

Figure 2.6 includes the amplification plot of a standard curve assay for B2M. This standard curve is analyzed in Figure 2.7 which plots cDNA dilution factor logarithmically against average $\mathrm{C}_{\mathrm{T}}$ value. Figure 2.7 graphs average $\mathrm{C}_{\mathrm{T}}$ versus cDNA dilution factor, where the slope of this line is equal to $\frac{-1}{\log E}$ and $E$ is the efficiency of the qPCR reaction. 


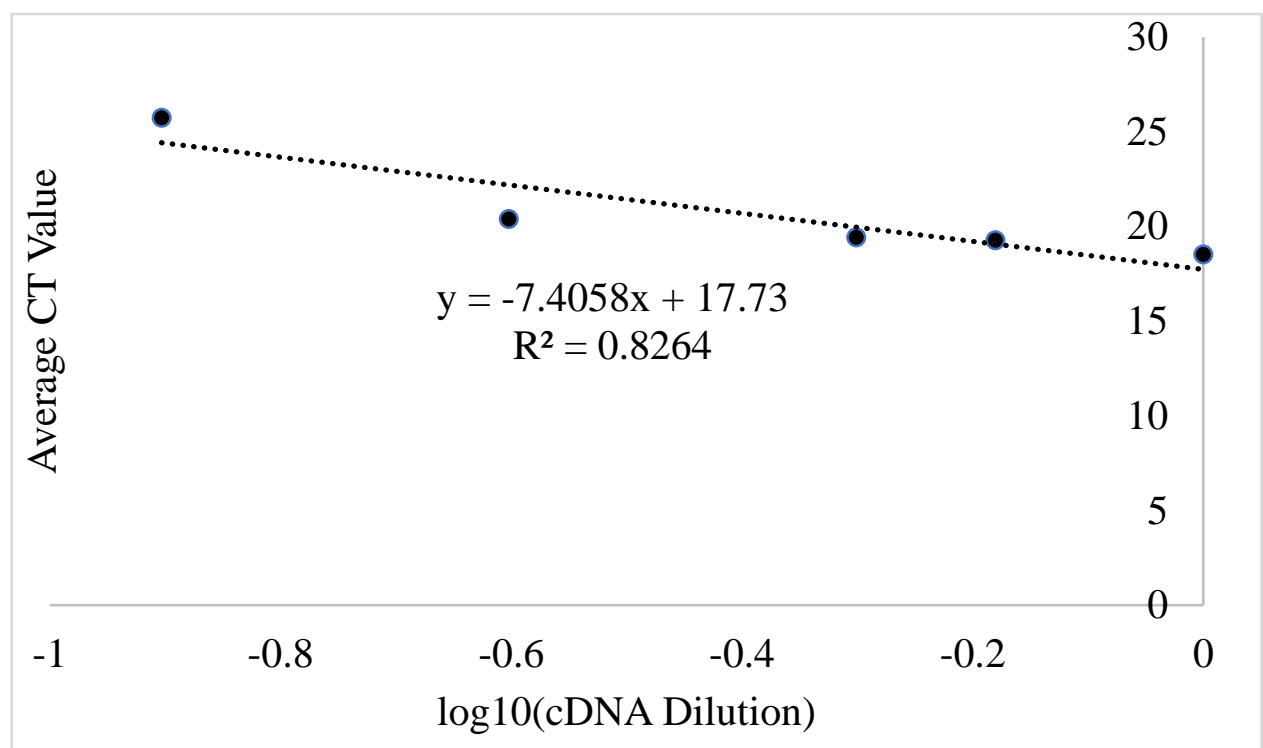

Figure 2.7: Representative Standard Curve Analysis. B2M cDNA dilution factor versus average $C_{T}$ value. Slope of the trendline is used to determine qPCR efficiency.

Genes of interest and their determined reaction efficiency by absolute quantification are listed in Table 2.3. Raw data and standard curves for CD36, HPRT1, and IL-6 are available in Appendix M.

Table 2.3: Measured Efficiencies by Gene from Standard Curve Analysis

\begin{tabular}{|l|l|l|}
\hline Gene & Slope $\mathrm{C}_{\mathrm{T}}$ vs. cDNA Dilution Factor & Efficiency (E) \\
\hline B2M & -7.4058 & 1.364675 \\
\hline CD36 & -10.493 & 1.245391 \\
\hline HPRT1 & -7.0971 & 1.383256 \\
\hline IL6 & -10.867 & 1.236009 \\
\hline
\end{tabular}

Testing the primers for replication efficiency revealed that none were within the ideal range of 1.8 to 2.0 [60]. These measurements prevented assumption of perfect efficiency, so comparisons using these gene-specific E values were utilized throughout this thesis rather than following convention to assume $\mathrm{E}=2$. 


\subsubsection{Relative Quantification}

\subsubsection{1 $E^{-C}$ Results}

$\mathrm{E}^{-\mathrm{C}} \mathrm{T}$ comparisons demonstrated that $\mathrm{B} 2 \mathrm{M}$ was an unsuitable reference gene due to decreased expression in the Moderate glucose condition and significantly increased expression in the High glucose condition. CD36 expression demonstrated no change due to treatment. HPRT1 expression decreased with Moderate glucose treatment but showed no change from Basal to High glucose treatment. IL-6 expression decreased under Moderate glucose treatment and increased significantly with High glucose concentration. Neither B2M or HPRT1 displayed consistent expression required of reference genes, so no suitable reference is available for these data. Figure 2.8 depicts $\mathrm{E}^{-\mathrm{C}_{\mathrm{T}}}$ comparisons. 
A

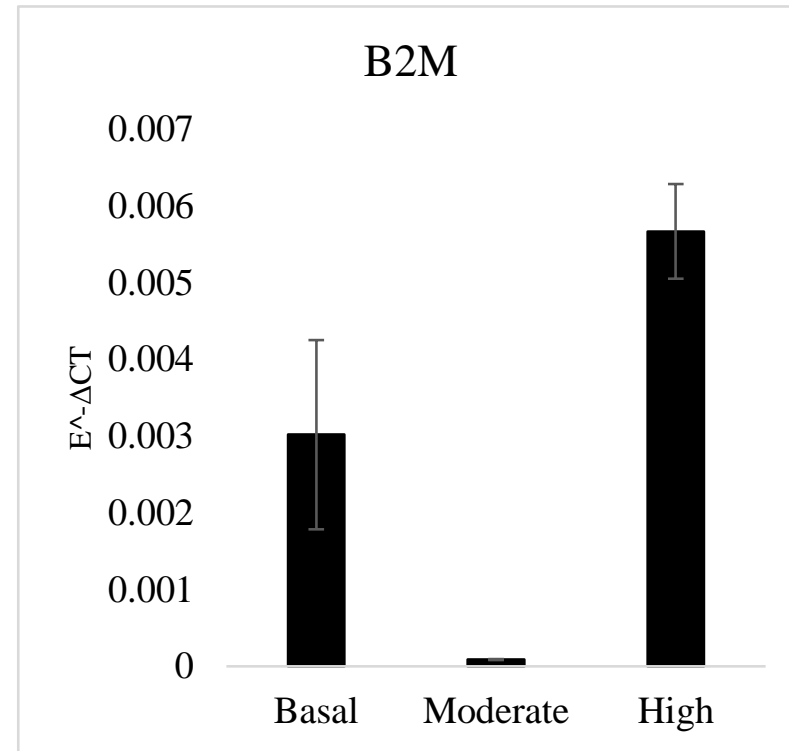

$\mathrm{C}$

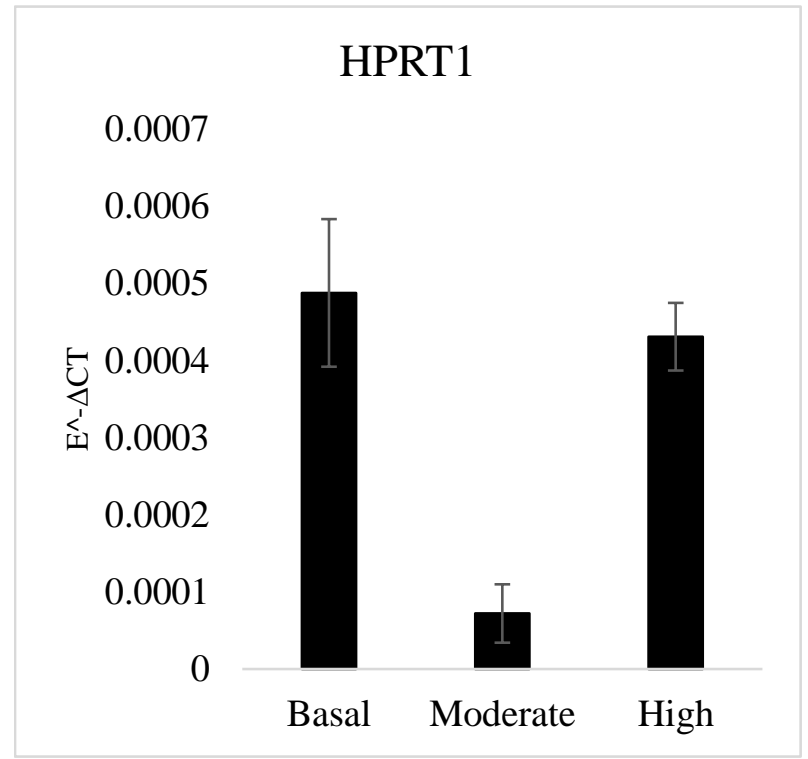

B
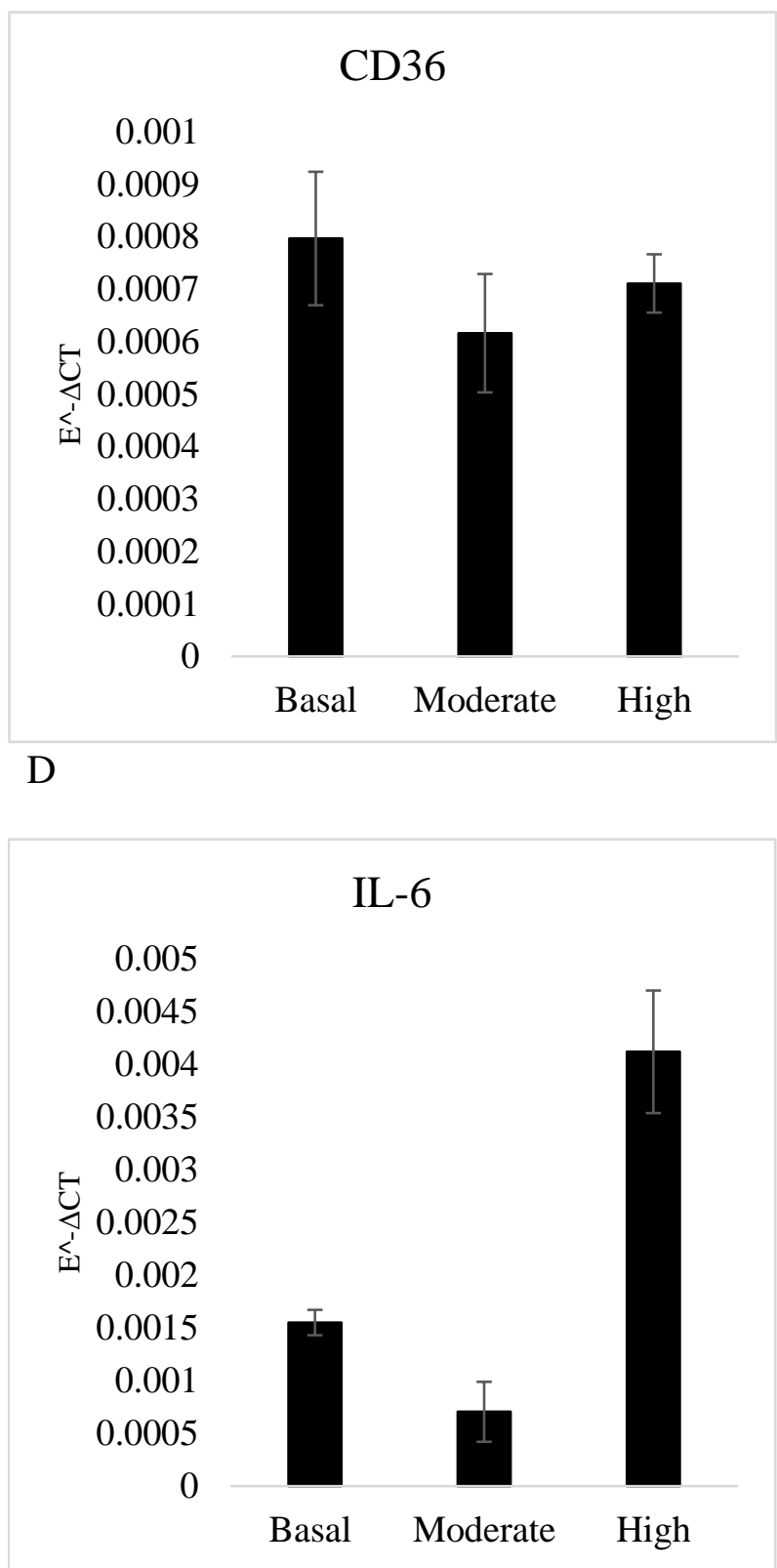

Figure 2.8: $E^{-C}$ T Analysis. (A) B2M expression decreases with Moderate treatment and increases with High glucose treatment. (B) CD36 expression remains consistent across treatments. $(C)$ HPRT1 expression decreases with Moderate treatment. (D) IL-6 expression increases with High glucose treatment. All values are in arbitrary units presented as mean \pm SEM. 
A lack of stable reference genes evident at this point prevented relative quantification methods based on normalization to a reference, namely $\mathrm{E}^{-\Delta \mathrm{C}_{\mathrm{T}}}$ (target-reference) and $\mathrm{E}^{-\Delta \Delta \mathrm{C}_{\mathrm{T}}}$ comparisons. This encompasses all comparisons across multiple genes. $\mathrm{E}^{-\Delta \mathrm{C}} \mathrm{T}$ (treated-untreated) comparisons between control and treated conditions within the same gene remained valid and appear in the following section.

\subsubsection{2 $E^{-\Delta C}$ Results}

Comparisons between control and treated samples using the $\mathrm{E}^{-\Delta \mathrm{C}}$ facilitated comparison of moderate and high glucose treatments within each gene. Expression of reference genes B2M and HPRT1 as well as target genes IL-6 and CD36 increased more with high glucose treatments compared to moderate glucose treatments. Figure 2.9 displays representative $\mathrm{E}^{-\Delta \mathrm{C}} \mathrm{T}_{\mathrm{results}}$, depicting an increase in abundance of all analyzed genes between Moderate and High glucose concentrations relative to Basal expression. 


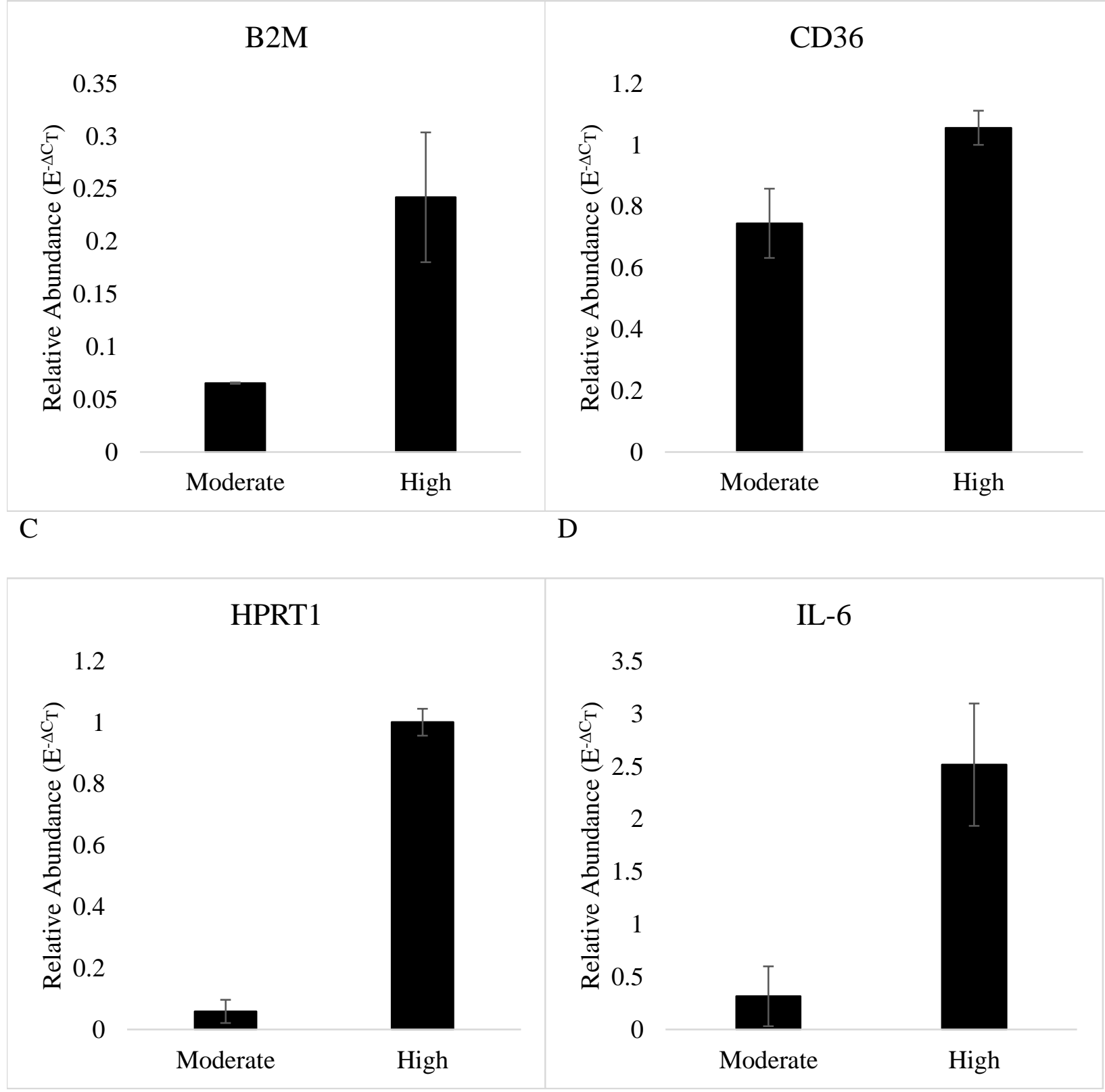

Figure 2.9: $E^{-\triangle C}$ Analysis of 7-Day Data. (A) B2M (B) CD36 (C) HPRT1 and (D) IL-6 expression all increased in High glucose condition compared to Moderate glucose condition relative to Basal. All values are in arbitrary units presented as mean \pm SEM. 


\subsubsection{3 $E^{-\Delta \Delta C}$ Results}

Due to inconsistent expression of reference genes across treatments, the $2^{-\Delta \Delta C}{ }_{T}$ method was previously determined to be inappropriate for analysis of data in this thesis. Rather than excluding this popular method entirely, the comparisons in Figure 2.10 were performed to demonstrate conclusions which could be improperly deduced from these data. Comparisons across multiple genes require assumption of a common reaction efficiency. For clarity, the common $\mathrm{E}=2$ assumption is utilized for this demonstration of methodology. CD36 expression was reported to have increased by approximately 170 -fold at the moderate glucose concentration, which was inconsistent compared to the unchanged expression shown in $\mathrm{E}^{-\mathrm{C}} \mathrm{T}$ analysis. IL-6 expression changes depending on the reference gene selected, with higher expression under the High glucose treatment relative to $\mathrm{B} 2 \mathrm{M}$ and under the Moderate glucose treatment relative to HPRT1. B2M and HPRT1 demonstrate opposite expression patterns relative to one another, implying that at least one of them is not an appropriate reference gene [63]. None of these conclusions are valid due to inconsistent reference gene expression. 


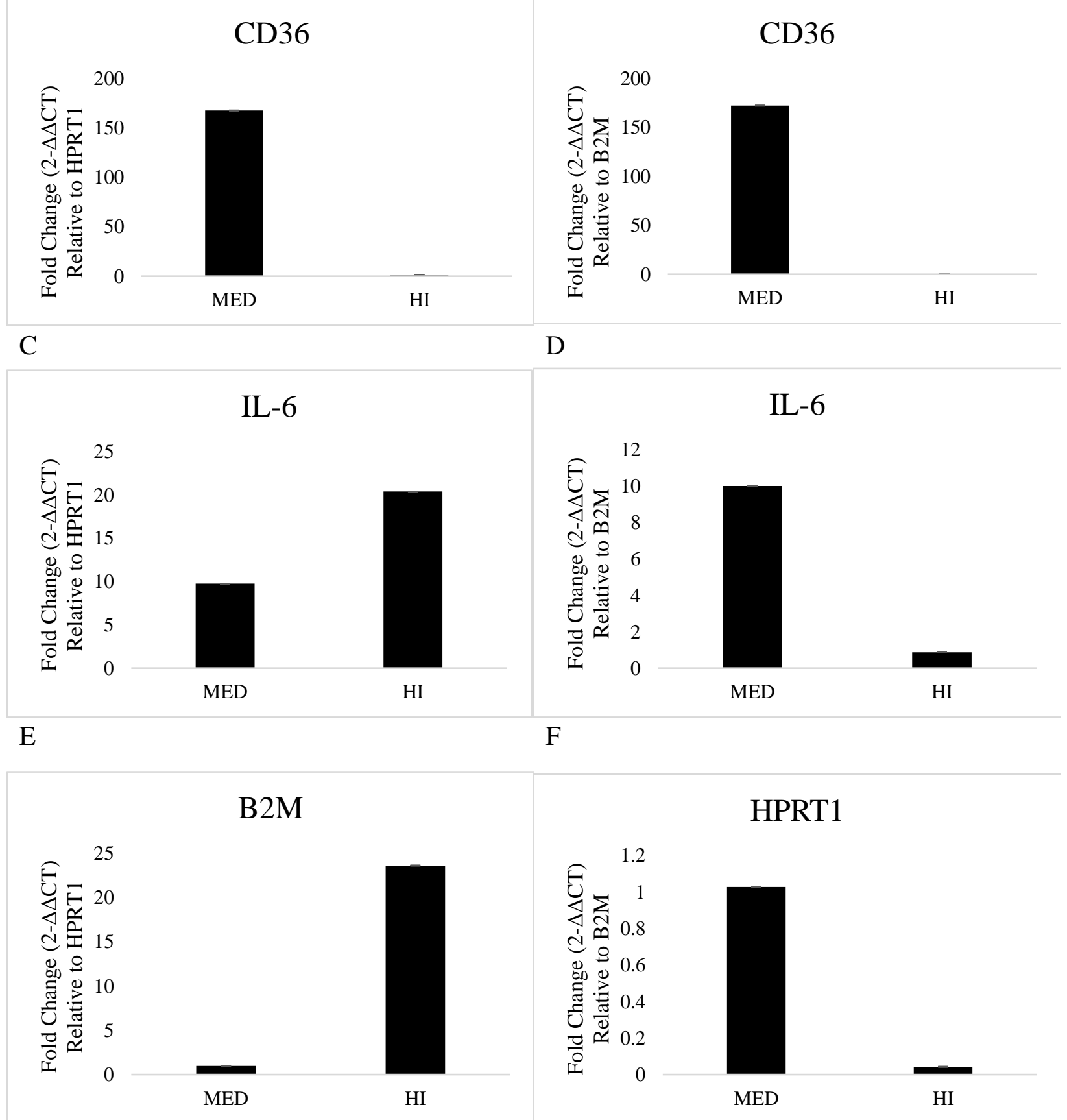

Figure 2.10: $2^{-\triangle \Delta C}$ T Analysis of 7-Day Data. Influence of reference gene on results of comparative $C_{T}$ method. Expression of genes $(A, C, E)$ relative to HPRT1 expression and $(B, D$,

$F$ ) relative to $B 2 M$ expression. All values are in arbitrary units presented as mean $\pm S E M$. 
Using the standard $2^{-\Delta \Delta C}{ }_{T}$ analysis relative to B2M resulted in conflicting IL-6 expression patterns compared to $2^{-\Delta \Delta C}$ Tanalysis relative to HPRT1. This demonstrated that at least one reference gene had inconsistent expression [63]. The stability of common reference genes B2M and HPRT1 across elevated glucose treatments cannot be assumed, although such an assumption may seem validated by their use as reference genes in literature [37]. The importance of analyzing reference genes before relying on their expression for normalization is therefore paramount. Because of evaluation and normalization of RNA concentration before cDNA synthesis, it remained valid to perform $2^{-\Delta \mathrm{C}}$ comparisons from untreated to treated conditions $[52,53]$.

\subsubsection{Probe-Quencher Primers}

Following data collection, it was realized that the products purchased from IDT were mistakenly PrimeTime Probes rather than PrimeTime Primers, indicating that in addition to strands designed to bookend target sequences during annealing, a probe with a fluorescent reporter and quencher pair was included. Probe sequences are available in Appendix A. The reporter molecule emits fluorescence when Taq DNA polymerase breaks the probe strand, separating the reporter from its quencher. The mechanism is depicted in Figure 2.11. 


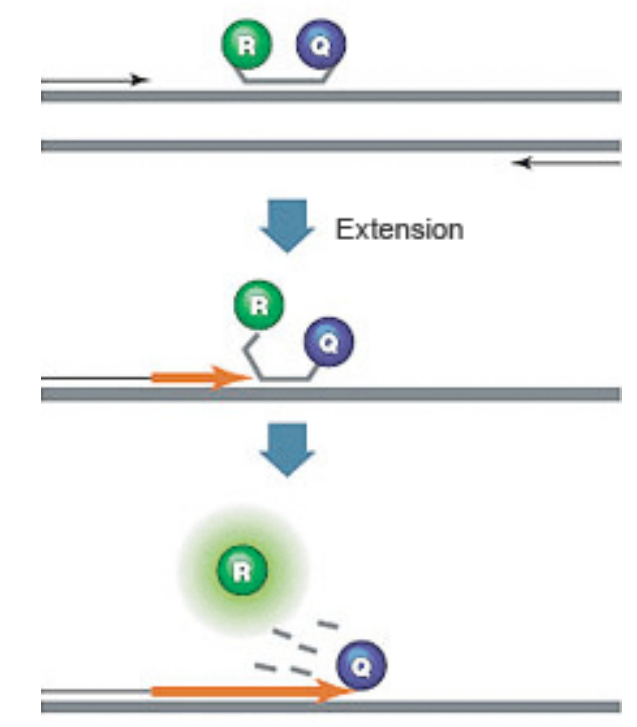

Figure 2.11: Probe-Quencher qPCR Reporting Mechanism

This addition increased the likelihood of unwanted PCR product formation as probe strands may have interfered with ideal replication. To validate results gathered with both fluorescent probes and SYBR intercalating dye, preliminary experiments performed for data analysis method selection were repeated without SYBR. Trials instead recorded fluorescence from the included probes.

Results from probe-only reactions displayed large variability and did not correspond with trends visible in samples evaluated using SYBR reporting. Many wells failed to report any fluorescence and noisy measurements required exclusion of additional wells, increasing error values. B2M, HPRT1, and IL-6 showed no meaningful changes in relative abundance with varying glucose concentration. CD36 showed higher relative abundance at the moderate concentration compared to basal but no change at the high glucose concentration relative to basal. Figure 2.12 depicts relative expression of B2M, CD36, HPRT1, and IL-6 as measured by probe and quencher reporting. 


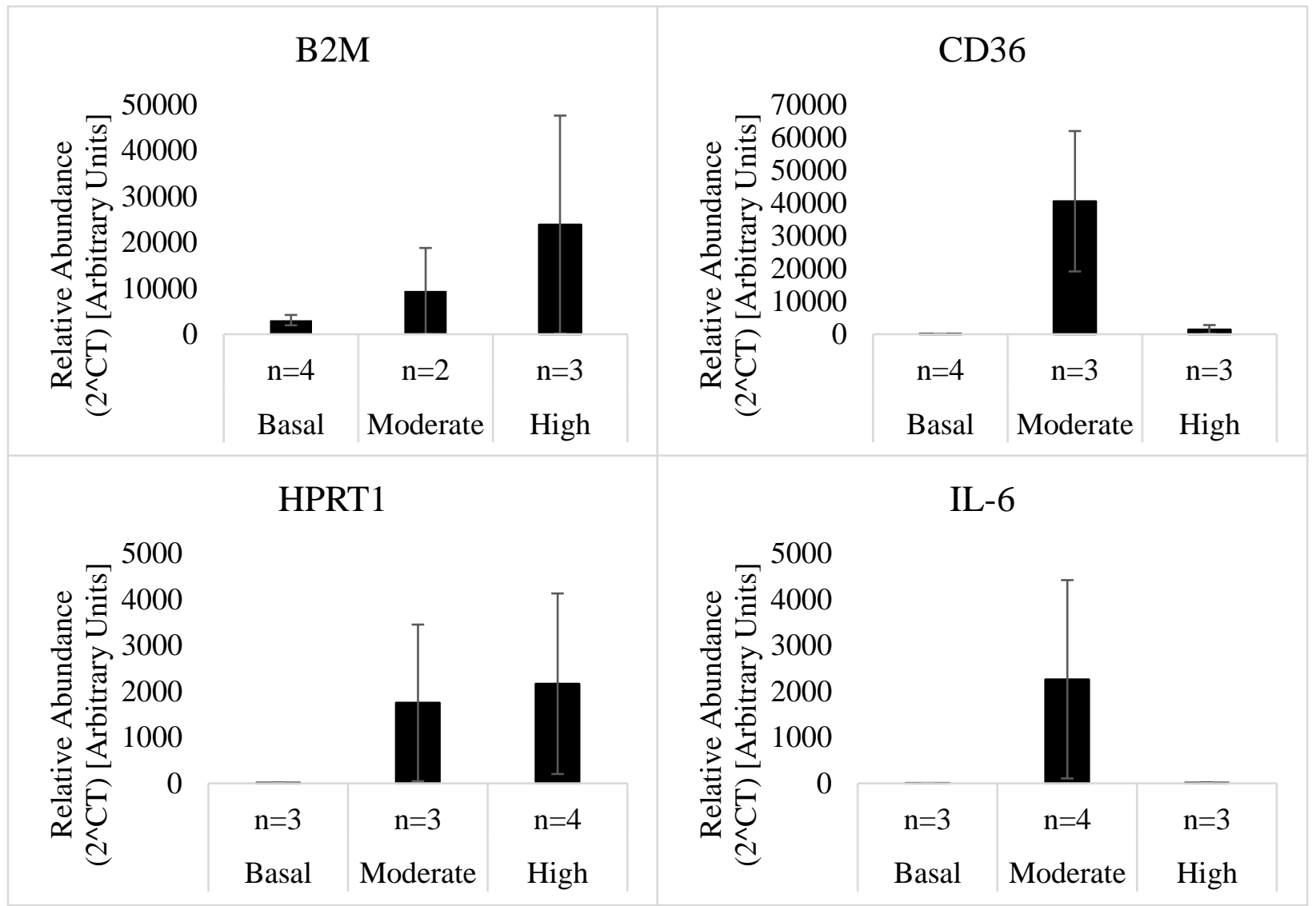

Figure 2.12: Probe-Quencher Reporting of 7-Day Responses. Data are presented as mean \pm

SEM.

Results from probe-quencher trials displayed massive variability within treatment groups.

No trends were replicated from SYBR green results to probe-quencher results, meaning that these experiments failed to validate results visible with SYBR fluorescence. Inclusion of probes likely reduced reaction efficiency substantially, consistent with measurements by absolute quantification. This necessitated use of measured $\mathrm{E}$ values rather than assuming perfect efficiency.

\subsubsection{Data Analysis Summary}

Measured efficiency values can be substituted for assumed $\mathrm{E}$ in relative quantification

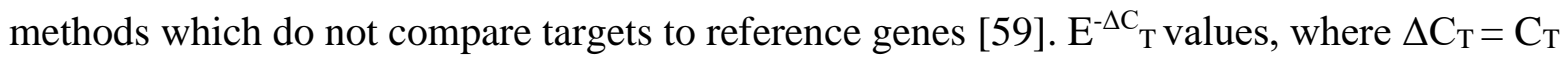




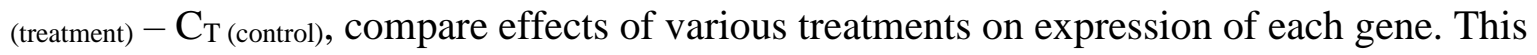
method was used throughout subsequent experiments.

\subsection{Discussion}

The goal of this first aim was to establish targets for gene expression and to select a method for qPCR data analysis based on preliminary data. Genes of interest IL-6 and CD-36 were selected from genome-wide association studies [33-35] to represent relevant pathway activity. Reaction efficiency (E) was determined by absolute quantification for each gene. Potential reference genes analyzed by $2^{-\mathrm{C}} \mathrm{T}$ demonstrated too much variability across treatment groups for use as an internal reference. This variability prohibits use of the most powerful comparative $\mathrm{C}_{\mathrm{T}}$ method, $2^{-\Delta \Delta \mathrm{C}} \mathrm{T}$ comparison. For this reason, qPCR data in subsequent experiments had to be analyzed by comparing $\mathrm{E}^{-\Delta \mathrm{C}}$ values for each gene between treatment groups and basal groups.

Low efficiency determined by absolute quantification was inconsistent with expectations of preverified primers. According to Scientific Support staff at the manufacturer IDT, this is most likely due to the mistaken ordering of primers with probe-quencher reporters. Scientific Support also supplied the information that due to the preverified nature of these probe-quencher primer sets, the desired qPCR products would still be amplified. Theoretical understanding supports conclusions made from an intercalating dye regardless of probe presence, and is supported by concurrent use of intercalating dyes and probes in literature [25-28]. Confidence in conclusions made from data gathered while using these primers is decreased by conflicting results seen with and without SYBR reporter, however this discrepancy is likely due to damage inflicted on the probe-quencher strand's fluorescent molecules by a year of improper storage. Fluorescent probes are light-sensitive while primers alone are not, so storage methods for 
standalone primers provide inadequate protection from UV rays. Had the probes been protected from light during storage, results likely would replicate those seen with SYBR reporting.

Lack of consistent B2M expression was unanticipated, but increased serum B2M levels are correlated with mortality in diabetic nephropathy [69] which may be related to increased B2M mRNA abundance in high glucose conditions. HPRT1 is commonly cited as a potential reference gene for investigations of elevated glucose. [43, 51]. Inconsistency in expression of this gene may therefore be due to differences in RNA quality across treatment groups.

This study was limited by the lack of stable reference genes, meaning fold change conclusions were impossible. Utilizing the $\mathrm{E}^{-\Delta \mathrm{C}}$ method maximizes the conclusions we can make without stable reference genes, but determination of a group of stable reference genes is a priority for future studies.

This aim contributes specific methods for design and selection of primers for qPCR analysis as well as a variety of data analysis protocols and the experiments for which each are valid. Genes of interest CD36 and IL-6 were selected along with reference genes B2M and HPRT1. In light of limitations due to improper primer ordering and lack of consistent reference gene expression, this aim selected $\mathrm{E}^{-\Delta \mathrm{C}_{\mathrm{T}}}$ (treatment-control) values for comparison of the effects of different treatments on CD36 and IL-6 expression. With this foundation in place, data collection began. 


\section{EVALUATING GENE EXPRESSION IN RESPONSE TO INCREASED EXTRACELLULAR GLUCOSE}

\subsection{Introduction}

The previous chapter identified genes of interest and determined the most appropriate data analysis method for use with probe-quencher primers and without consistent reference gene expression. With this foundation, effects of glucose concentration and treatment duration were investigated. As discussed in Chapter 1, glucose concentrations (5.5 mM, 10.5 mM, and 25.5 $\mathrm{mM}$ ) were selected to represent the progression of diabetic macrovascular disease. In an effort to represent disease evolution, effect of treatment duration on expression of CD36 and IL-6 was evaluated by qPCR. Elevation in relative expression of CD36 and IL-6 under elevated glucose at these timepoints would be consistent with the acute cellular response to hyperglycemia discussed in Chapter $1[5,24,59]$. This Chapter encompasses Aim 2 of this thesis: to evaluate acute changes in gene expression, specifically of IL-6 and CD36 in HUVECs, due to increased extracellular glucose.

\subsection{Methods}

In fulfillment of this aim, HUVECs were cultured at basal, moderate, and high glucose concentrations for 7 or 14 days. These timepoints were selected to represent early diabetic macrovascular disease progression [6] and are supported by previously published studies using similar timepoints to investigate cellular responses to elevated glucose [3-5]. Cells were cultured in media according to treatment before RNA was isolated and transcribed to complementary cDNA. cDNA was used in RT-qPCR to evaluate relative expression with data analysis techniques as described in Chapter 2 and further summarized below. 


\subsubsection{Cell Culture}

Human Umbilical Vein Endothelial Cells were obtained from Lonza Walkersville Inc. (Catalog number 2519A, LOT 0000470896) and cultured in EGM-2 Growth Media (Catalog number 3162), also from Lonza. Due to product shortages, EGM-Plus Growth Media (Catalog number 5035) with supplemental L-Glutamine (VWR, Catalog number 16777-162) at a final concentration of $10 \mathrm{mM}$ was substituted when EGM-2 Growth Media was unavailable. Cells were cultured at 5\% CO2(manually measured daily with Fyrite Gas Analyzer), 80\% relative humidity and $37^{\circ} \mathrm{C}$. Cell culture media was changed every 48-72 hours throughout culture and all experiments were conducted on cells subcultured from the same source vial at passage 5 . Culturing took place in T-75 flasks passaged at a 1:3 ratio and were $80-100 \%$ confluent at the time of RNA isolation, yielding approximately 6-8 million cells.

Induction of high glucose conditions consisted of adding $80 \mathrm{mM}$ sterile glucose solution (VWR Catalog number 97062-880) to final concentrations of $10.5 \mathrm{mM}$ and $25.5 \mathrm{mM}$ glucose, as described in Chapter 1. Detailed protocols for cell thawing, passing, feeding, and freezing can be found in Appendix E.

\subsubsection{RNA Isolation}

In order to analyze relative expression of certain genes, total RNA was isolated from cells using RNEasy Mini Kit (Qiagen Catalog number 74106). Once T-75's reached 80-100\% confluence, $3 \mathrm{~mL}$ trypsin (Sigma, T3924) cleaved cells from culture vessels. Centrifugation and aspiration of trypsin and cell media was performed before cells were resuspended in PBS (ThermoFisher, 14190359) and flash-frozen in liquid nitrogen.

To begin RNA isolation, cells were first chemically lysed in a buffer containing chaotropic salts to release contents before being transferred to a biopolymeric spin column 
(QIAshredder, Qiagen) to further homogenize cell lysate. Lysate was further separated by centrifugation through silica spin columns. Silica immobilized nucleic acids while allowing lipids, proteins, and cell fragments to pass through. RNA remained bound to silica through two washing steps which removed DNA and other residual contaminants by first modifying the salt concentration to make DNA insoluble and second washing with ethanol to remove salt. RNA was released from silica in Milli-Q pure water due to intermolecular interactions of RNA molecules and water molecules.

RNA was then assessed for purity by ultraviolet-visible (UV-Vis) spectrophotometry using a NanoDrop 2000 (ThermoFisher). This device measures the absorbance of isolated RNA samples at $260 \mathrm{~nm}$ to determine concentration using the Beer-Lambert equation and measures absorbance at $280 \mathrm{~nm}$ to determine purity by comparing absorbance at $260 \mathrm{~nm}$ over absorbance at $280 \mathrm{~nm}$. RNA with a 260/280 ratio of 1.95-2.05 can used to transcribe cDNA. Detailed protocols for RNA Isolation and RNA Purity Assessment can be found in Appendix F.

\subsection{3 cDNA Synthesis}

Following isolation, RNA was transcribed into complementary DNA (cDNA) by reverse transcription using iScript cDNA Synthesis Kit (BIO-RAD, Catalog number 1708890). The kit uses both single-stranded sequences of deoxythymine nucleotides (oligo-dT) and random sequences of six nucleotides (random hexamer) primers to bind template strands of RNA. Reverse transcriptase binds double-stranded primed segments of RNA and synthesizes cDNA which is subsequently amplified during qPCR. Detailed protocols for cDNA synthesis can be found in Appendix G.

cDNA was combined with primers, probes, Taq polymerase, dNTPs, SYBR, and enhancers for qPCR. Recall from the previous chapter that probes were mistakenly purchased 
with primers and should not be included in the future. qPCR proceeded according to methods described in the previous chapter. Briefly, reaction mixes were cyclically heated and cooled in a thermocycler equipped to record fluorescence of SYBR green intercalating dye. Fluorescence increased predictably over 40 cycles of qPCR, facilitating determination of a $\mathrm{C}_{\mathrm{T}}$ value representative of starting mRNA concentration. $\mathrm{C}_{\mathrm{T}}$ values were compared by $\mathrm{E}^{-\Delta \mathrm{C}} \mathrm{T}$ or "Measured Efficiency" as described in the previous chapter. To demonstrate the importance of measuring efficiency by absolute quantification, comparisons using the prevalent assumption of perfect efficiency (E=2) or "Assumed Efficiency" were also conducted.

Gene expression was compared by qPCR for genes of interest CD36 and IL-6 as well as reference genes B2M and HPRT1. Due to inconsistent reference gene expression across treatment groups, comparisons to reference gene expression were not conducted. Multiple experiments were conducted, each evaluating HUVEC responses to three concentrations of glucose in cell culture media. Basal $(5.5 \mathrm{mM})$ acted as a control while Moderate $(10.5 \mathrm{mM})$ and High (25.5 mM) concentrations induced varying degrees of elevated glucose conditions. An overview of experimental time points and the number of cell culture flasks evaluated, or "Independent Trials" as well as replicate qPCR samples, or "Technical Replicates" is provided in Table 3.1. In this table and throughout this chapter, evaluations are listed alphabetically by gene name for convenience.

Differences between groups were assessed by ANOVA of $\mathrm{C}_{\mathrm{T}}$ values in JMP [72]. ANOVA comparisons can be found in Appendix L. Data are presented as mean \pm standard error. 
Table 3.1: Experimental Design for qPCR Experiments

\begin{tabular}{|c|c|c|c|c|c|}
\hline & & $\begin{array}{c}\text { Independent } \\
\text { Trials }\end{array}$ & $\begin{array}{c}\text { Technical } \\
\text { Replicates }\end{array}$ & $\begin{array}{c}\text { Sample } \\
\text { Size (n) }\end{array}$ \\
\hline \multirow{3}{*}{$\begin{array}{c}\text { Replicates } \\
\text { Evaluated for } \\
\text { Each } \\
\text { Treatment }\end{array}$} & 7 Day & B2M & 2 & 3 & 6 \\
\cline { 3 - 6 } & & CD36 & 3 & 3 & 9 \\
\cline { 3 - 6 } & 14 Day & HPRT1 & 3 & 3 & 9 \\
\cline { 3 - 6 } & & IL-6 & 3 & 3 & 6 \\
\cline { 3 - 6 } & & CD36 & 2 & 3 & 6 \\
\cline { 3 - 6 } & & HPRT1 & 2 & 3 & 6 \\
\cline { 3 - 6 } & & IL-6 & 2 & 3 & 6 \\
\hline
\end{tabular}

\subsection{Results}

When evaluated at seven days, relative expression of B2M decreased compared to the basal condition at the moderate glucose concentration while expression increased in the high glucose concentration. CD36 expression remained low and relatively consistent across treatment groups. Relative expression of HPRT1 decreased with moderate glucose treatment and was consistent at the high glucose concentration relative to the basal glucose treatment. Expression of IL-6 showed no change at the moderate glucose concentration and increased at the high glucose concentration $(\mathrm{p}<0.001)$. Assumed efficiency values used $E=2$ while measured efficiency values used efficiencies determined for each gene in Chapter 2, which are repeated below in Table 3.2.

Table 3.2: Measured Efficiency Values from Chapter 2

\begin{tabular}{|l|l|}
\hline Gene & Efficiency (E) \\
\hline B2M & 1.364675 \\
\hline CD36 & 1.245391 \\
\hline HPRT1 & 1.383256 \\
\hline IL-6 & 1.236009 \\
\hline
\end{tabular}


Figure 3.1 depicts relative abundance $\left(\mathrm{E}^{-\mathrm{C}} \mathrm{T}\right)$ of $\mathrm{B} 2 \mathrm{M}, \mathrm{CD} 36$, IL-6, HPRT1, and CD36 after seven days of treatment at $5.5 \mathrm{mM}$ (Basal), $10.5 \mathrm{mM}$ (Moderate), or $25.5 \mathrm{mM}$ (High).

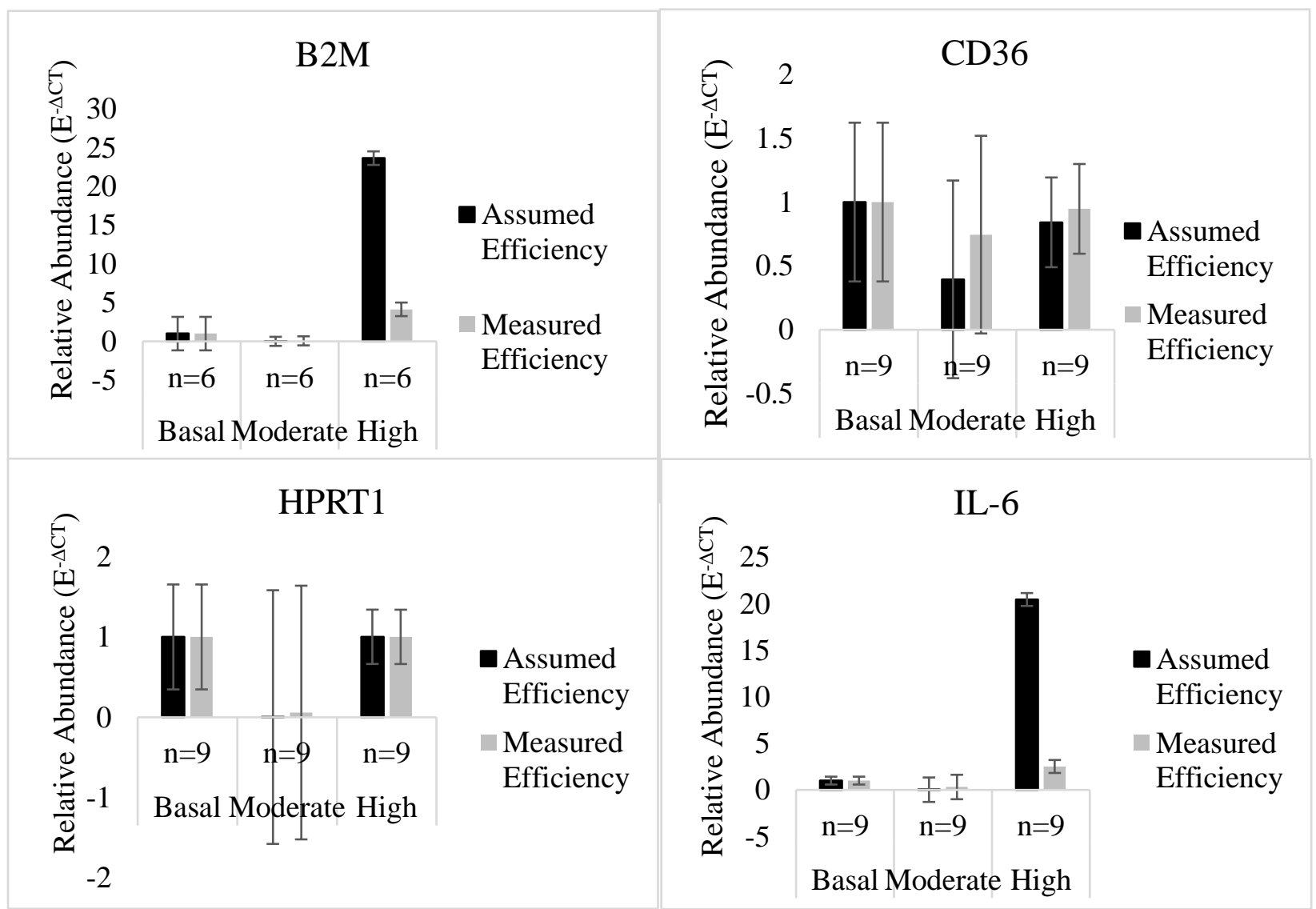

Figure 3.1: Relative Abundance Comparisons Between Assumed and Calculated Efficiency

Values after 7 Day Treatment. (A)B2M expression increased at High glucose concentration. (B) CD36 expression remained consistent across treatment groups. (C) HPRT1 expression remained consistent across treatment groups. (D) IL-6 expression increased at High glucose concentration. Data are presented as mean \pm SEM.

Evaluation of relative gene expression at 14 days was consistent with results at seven days. B2M expression increased at the highest glucose concentration as did relative expression of IL-6 (p<0.001). HPRT1 expression was more consistent across treatment groups at fourteen days 
than seven days. CD36 displayed very low expression and relatively consistent expression across treatment groups. Figure 3.2 shows relative expression of gene targets after 14 days of treatment.

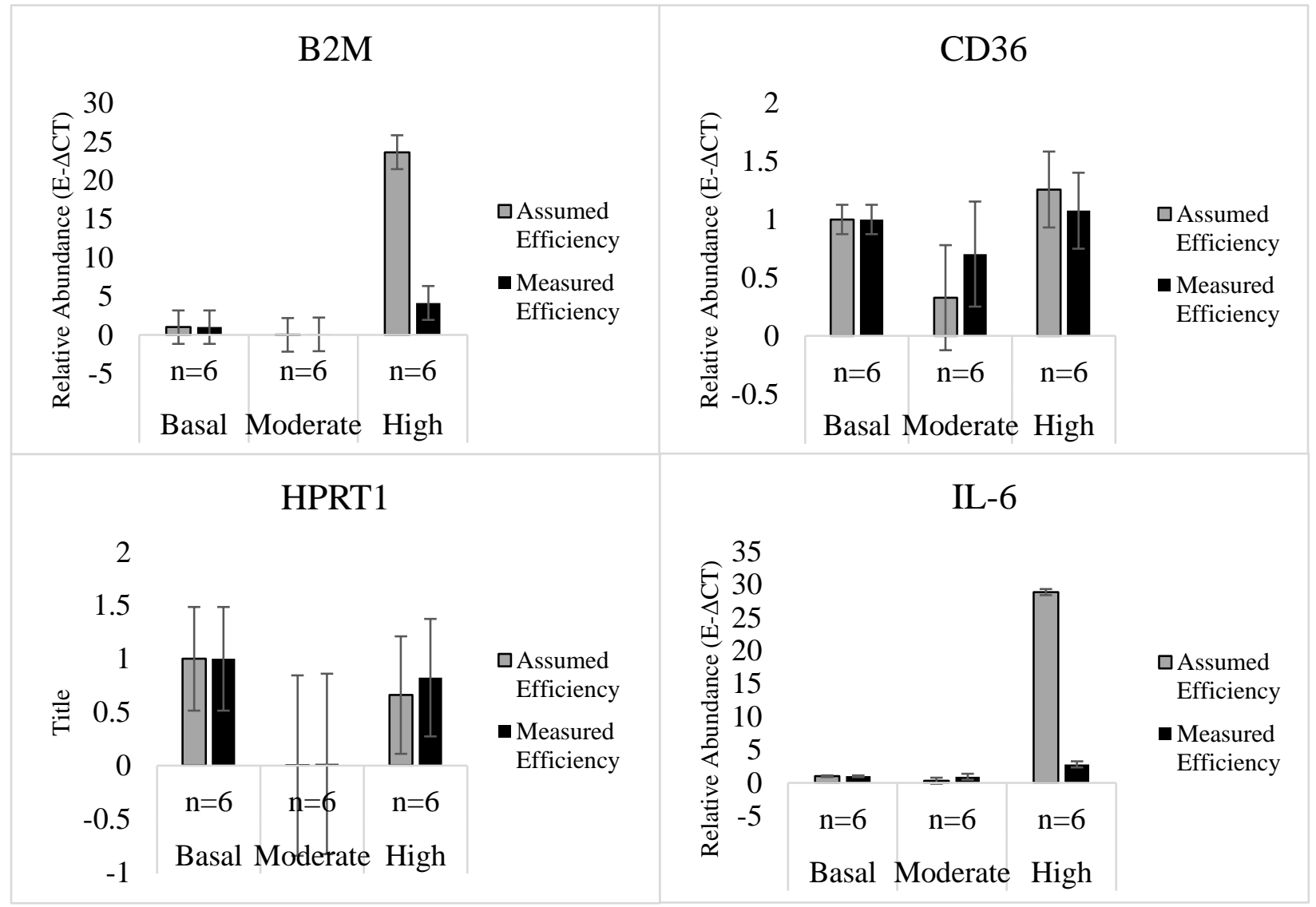

Figure 3.2: Relative Abundance of Target Genes at 14-day Timepoint. (A) B2M expression increased with High glucose treatment. (B) CD36 expression was unaffected by glucose treatment. (C) HPRT1 expression decreased with Moderate glucose treatment but remained consistent from Basal to High glucose treatment. (D) IL-6 expression increased with High glucose treatment. All data are in arbitrary units and are presented as mean \pm SEM.

\subsection{Discussion}

This aim sought to compare gene expression results between basal and elevated glucose conditions at seven and fourteen days. Expression of mRNA for cytokines CD36 and IL-6 was analyzed by qPCR. CD36 expression remained consistent across elevated glucose treatments 
indicating that either treatment intensity or treatment duration may be insufficient to induce CD36 upregulation in HUVECs with high glucose culture. This finding was inconsistent with documented work showing increased CD36 expression with elevated glucose treatment in human microvascular endothelial cells, however these evaluations were performed following 24 hours of treatment [31]. Dose- and duration-dependent PKC upregulation following glucose treatment of HUVECs for 7 and 14 days [73] implies upregulation of both CD36 and IL-6 through connected pathways [50, 66, 67]. Differential expression of CD36 across various endothelial cell sources is the most likely explanation for low CD36 expression regardless of treatment. Macrovascular ECs, including HUVECs, have substantially lower CD36 expression than microvascular endothelial cells (MVECs) [76]. HUVECs were characterized as lacking surface CD36 expression [77], suggesting low CD36 mRNA production. This validates low levels of expression detected in this thesis, offering support to these analysis methods. While analysis of CD36 expression in HUVEC under high glucose conditions offers no insight into accuracy of the diabetic macrovascular disease blood vessel mimic model, CD36 expression could be used in the future to evaluate alignment of BVM expression with macrovascular vessel expression.

IL-6 mRNA abundance was found to increase in HUVECs treated with $25.5 \mathrm{mM}$ glucose at 7 and 14 days, which is consistent with gene expression in Type 2 Diabetes [70, 71]. IL-6 upregulation appeared similar in magnitude to upregulation of B2M, however without a consistently-expressed reference gene there is no valid method by which to compare expression of B2M and IL-6 [60]. Though B2M was initially included as a potential reference gene, as mentioned briefly in the previous chapter, serum B2M levels are correlated with mortality from diabetic vascular diseases [69]. Though increased B2M mRNA expression under high glucose conditions has yet to be described, this may partially account for its increase. While lacking a 
control may limit the conclusions drawn from these data, the trends present suggest that the documented increase in B2M expression is genuine and should disqualify B2M for use as a reference gene in elevated glucose condition studies.

The lack of a stable reference gene and a common reaction efficiency required that IL-6 upregulation be evaluated only in comparison to the untreated control. IL-6 expression increased by approximately $250 \%$ at 7 and 14 days in $25.5 \mathrm{mM}$ glucose, which correlates well with a significant increase in HUVEC IL-6 mRNA expression after 14-day treatment at $20 \mathrm{mM}$ glucose compared to $5 \mathrm{mM}$ as evaluated by Northern blotting [79]. While lack of a stable reference gene also limits conclusions possible from these data, consistency with previously documented work supports that upregulation of IL-6 following implementation of increased extracellular glucose conditions is valid. This finding supports the hypothesis that oxidative stress can be detected following administration of supplemental glucose by assessing IL-6 mRNA expression in HUVECs.

mRNA expression of all genes was reduced under the Moderate glucose treatment. This trend is inconsistent with signaling cascade-based predictions and was visible in all samples, raising doubts about its validity. All samples in each treatment group were transcribed from RNA to cDNA simultaneously, suggesting that experimental error may have caused all of these transcription reactions to fail. Failure to include enough Reverse Transcriptase in these reactions would yield low cDNA, accounting for the very low expression in every gene investigated. This means that conclusions cannot be made from this model about the effect of $10.5 \mathrm{mM}$ glucose treatment on mRNA expression, however conclusions involving basal (5.5 mM) and high (25.5 $\mathrm{mM}$ ) glucose treatment groups remain valid. 
Low qPCR reaction efficiency challenges confidence in these results, as mentioned in the previous chapter. It was determined that low reaction efficiency was due to mistaken ordering of probe-quencher primers. The preverified nature of these primers ensures desired PCR products are amplified regardless of poor efficiency and that though unwanted products may be formed due to probe-quencher inclusion, they cannot be exponentially amplified. This is further validated by evaluation of dissociation curves mentioned in Chapter 2. Heating PCR products after amplification demonstrated approximate uniformity in the dissociation curves of each sample, suggesting similar size and composition. This supports results calculated using measured efficiency values. In the larger context of this thesis, these data support supplemental extracellular glucose as a component of a diabetic macrovascular disease model as measured by acute upregulation of IL-6. Future work based on these findings will be further discussed in Chapter 5. 


\section{EVALUATING ROS GENERATION IN RESPONSE TO INCREASED EXTRACELLULAR GLUCOSE}

\subsection{Introduction}

Chapter 2 established a methodology for gene expression analysis. Chapter 3 focused on measuring gene expression in response to elevated glucose at 7 and 14 days of treatment. Next, evaluation of Reactive Oxygen Species (ROS) generated at 24 and 48 hours was performed in an attempt to capture a more acute aspect of the cellular response to high glucose concentrations. ROS is generated more immediately than mRNA expression changes, permitting a shorter treatment duration, though ROS production remains elevated throughout exposure to high glucose [80]. Elevated glucose at $25 \mathrm{mM}$ has been shown to gradually increase ROS generation in bovine aortic endothelial cells following three to 24 hours of treatment [81]. ROS overproduction and decreased DNA synthesis were detected in HUVECs treated with $28 \mathrm{mM}$ glucose for 48 hours [82]. This aim evaluated oxidative stress responses to short-term elevated glucose treatment by quantifying ROS generation and cell viability at 24 and 48 hours.

Oxidative stress induced by extracellular hyperglycemia causes endothelial damage in two ways: by decreasing available antioxidants and by production of reactive oxygen species (ROS). ROS upregulate formation of advanced glycation end products and activate the protein kinase $\mathrm{C}$ (PKC) pathway [35]. This asserts that upregulation of IL-6 detected at 7 and 14 days by qPCR previously in this thesis can be connected by known signaling pathways to overproduction of ROS at the same glucose concentrations. Figure 4.1 depicts the process from transport of glucose through the endothelial cell membrane to tissue damage. Detection of ROS in endothelial cells reflects increased endothelial damage and associated macrovascular injury [ 34 , $61,62]$. 


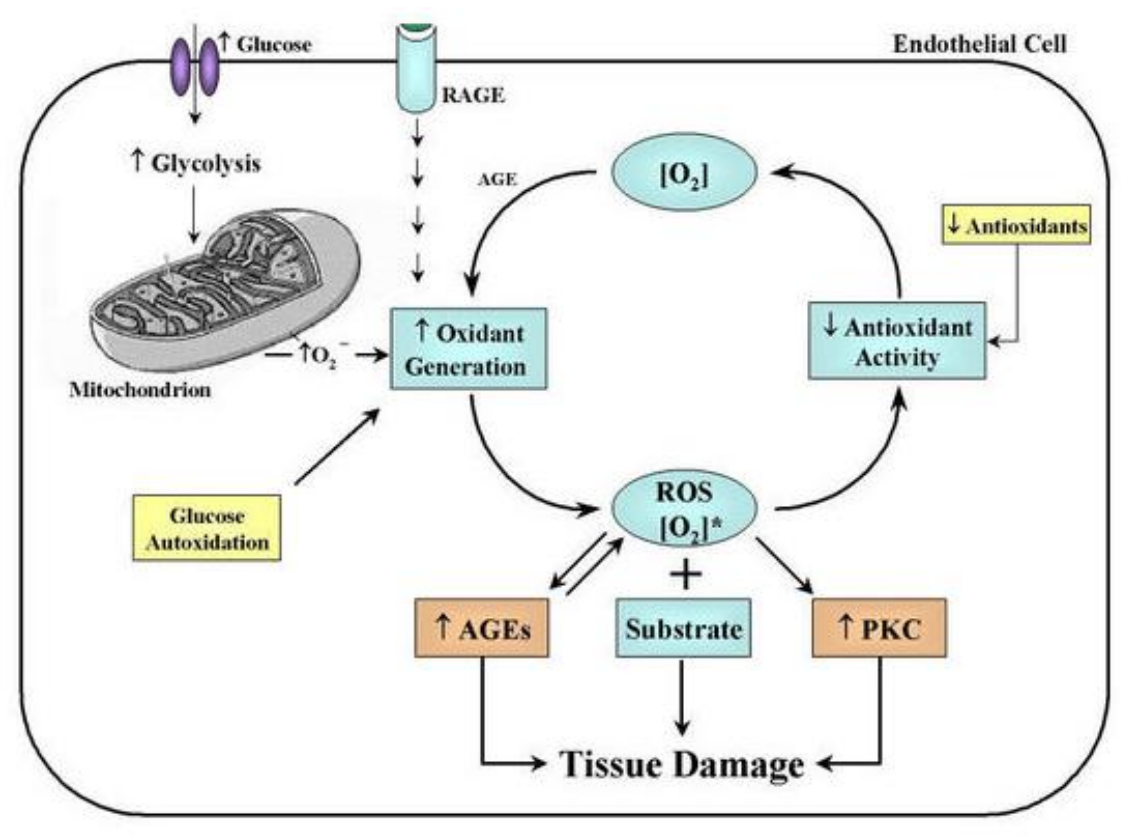

Figure 4.1: Increased Extracellular Glucose Increases ROS Generation and Subsequent Tissue

Damage [35]. Glucose transport initiates glycolysis which increases oxidant generation.

Reactive Oxygen Species (ROS) are a byproduct of oxidant generation and induce tissue damage by increasing Advanced Glycation End-products (AGEs) and upregulating the PKC pathway.

Evaluations of ROS generation in this chapter build upon previous experiments by detecting imbalances in cellular oxidation and antioxidation. This imbalance is termed oxidative stress, a precursor to endothelial dysfunction and diabetic macrovascular disease [14]. As previously mentioned, ROS produced during oxidative stress upregulate $\mathrm{PKC}$ and contribute to AGE formation [35]. IL-6 is directly upregulated by PKC and indirectly upregulated by AGE formation, associating increased ROS with increasing IL-6 mRNA expression [51, 66]. Additionally, measuring cell viability in response to elevated glucose is a direct metric of progression of endothelial dysfunction because apoptosis is upregulated as oxidative stress worsens $[9,10]$. Decreasing cell viability in vitro under high glucose conditions has been 
documented [82], so replication of these results would establish confidence in this imaging method.

Following validation of these methods, fluorescent staining evaluations for oxidative stress and endothelial dysfunction could be applied to blood vessel mimics. Visual methods for assessing ROS generation and cell viability in a diabetic BVM following device implantation would contribute to determining how well the diabetic BVM models replicate diabetic macrovascular disease conditions where ROS generation is high and cell viability is decreased $[28,78]$.

\subsection{Methods}

In order to evaluate ROS generation due to elevated glucose, HUVECs were cultured in basal, moderate, and high glucose concentration medias for 24 or 48 hours in cover glassbottomed eight well plates according to methods described below. Fluorescent staining indicated the presence of ROS and was captured and quantified by confocal microscopy.

Imaging experiments were conducted using a Confocal Laser Scanning Microscope (Fluoview 1000, Olympus). Confocal microscopy uses lasers and optical filters to emit light at a specific wavelength suited to excite fluorophores of a given dye. When excited at the correct wavelength, fluorophores emit light at a predictable lower wavelength. This light is isolated with optical filters, and photons are then counted by the microscope's photo multiplier tube. The number of photons counted at each pixel location is assembled into an image. In these experiments, widefield imaging was used to locate the center of each well, and then a $20 \mathrm{X}$ magnification Z-stack was taken for each sample. 


\subsubsection{Experimental Design}

The methods described above were carried out in two experiments. The first experiment assessed the effect of treatment duration. The second experiment attempted to isolate the effect of elevated glucose and ROS overproduction on cell viability by evaluating all samples after 48 hours of treatment [82]. Table 4.1 lists experiments with their treatment groups and staining concentrations.

Table 4.1: Experimental Design

\begin{tabular}{|c|c|c|c|}
\hline Experiment & Treatment & Sample Size & Staining Concentrations \\
\hline \multirow[t]{7}{*}{ Experiment 1} & Basal, 24 Hours & 4 & \multirow{7}{*}{$\begin{array}{l}\text { Hoechst: } 1 \mu \mathrm{M} \\
\text { EthD-1: } 1 \mu \mathrm{M} \\
\mathrm{H}_{2} \text { DCFDA: } 1 \mu \mathrm{M}\end{array}$} \\
\hline & Basal, 48 Hours & 7 & \\
\hline & Moderate, 24 Hours & 6 & \\
\hline & Moderate, 48 Hours & 5 & \\
\hline & High, 24 Hours & 6 & \\
\hline & High, 48 Hours & 5 & \\
\hline & Positive Control, 48 Hours & $2^{\ddagger}$ & \\
\hline \multirow[t]{4}{*}{ Experiment 2} & Basal, 48 Hours & 7 & \multirow{4}{*}{$\begin{array}{l}\text { Hoechst: } 2 \mu \mathrm{M} \\
\text { EthD-1: } 1 \mu \mathrm{M} \\
\mathrm{H}_{2} \text { DCFDA: } 1 \mu \mathrm{M}\end{array}$} \\
\hline & Moderate, 48 Hours & 8 & \\
\hline & High, 48 Hours & 8 & \\
\hline & Positive Control, 48 Hours & 8 & \\
\hline
\end{tabular}

+ Excluded from results due to low sample size

The first experiment yielded oversaturated images; adjustments to alleviate this were made for the second experiment. Table 4.2 lists dyes with their excitation and emission wavelengths as well as microscope settings for the first imaging experiment. 
Table 4.2: Dyes and Imaging Settings for Experiment 1

\begin{tabular}{|l|l|l|l|l|}
\hline Dye Name & $\begin{array}{l}\text { Excitation/Emission } \\
\text { Wavelength (nm) }\end{array}$ & Filter Used & $\begin{array}{l}\text { Laser } \\
\text { Setting (\%) }\end{array}$ & $\begin{array}{l}\text { High Voltage } \\
\text { Setting }\end{array}$ \\
\hline Hoechst 33342 & $350 / 461$ & DAPI & 18.0 & 700 \\
\hline $\begin{array}{l}\text { Carboxy- } \\
\mathrm{H}_{2} \text { DCFDA }\end{array}$ & $495 / 529$ & $\begin{array}{l}\text { Alexa } \\
\text { Fluor 488 }\end{array}$ & 15.0 & 700 \\
\hline $\begin{array}{l}\text { Ethidium } \\
\text { Homodimer }\end{array}$ & $528 / 617$ & $\begin{array}{l}\text { Alexa } \\
\text { Fluor 594 }\end{array}$ & 15.0 & 700 \\
\hline
\end{tabular}

Table 4.3 lists dyes and their imaging settings for Experiment 2. Laser power was

reduced substantially for EthD from the first experiment to the second while Hoechst laser power and voltage were increased in attempts to equalize saturation.

Table 4.3: Experiment 2 Imaging Settings

\begin{tabular}{|l|l|l|}
\hline Dye Name & Laser Setting (\%) & High Voltage Setting \\
\hline Hoechst 33342 & 20.0 & 800 \\
\hline Carboxy-H $\mathrm{H}_{2}$ DCFDA & 15.0 & 750 \\
\hline Ethidium Homodimer & 1.0 & 675 \\
\hline
\end{tabular}

\subsubsection{Cell Culture for Confocal Microscopy}

Cell culture for confocal imaging was performed as described for qPCR on specialized culture plates. HUVECs (Lonza, C2519A) were thawed into T-75 flasks and cultured in Lonza EGM-Plus cell media at $5 \% \mathrm{CO}_{2}$ and $80 \%$ relative humidity. Upon reaching 70 to $80 \%$ confluency, they were passed into cover glass-bottomed 8 well plates (Thermo Scientific, 155411 ) with a seeding density of approximately 2,500 cells per well. Passing occurred 72 hours before staining. Treatment of 48 -hour samples began 24 hours after seeding and treatment of 24- 
hour samples began 24 hours later. Cells were checked for morphological changes and confluency before each treatment. A detailed protocol for cell culture for confocal imaging is available in Appendix J.

\subsubsection{ROS Generation Assessment}

The Image-IT LIVE Green Reactive Oxygen Species Detection Kit (ThermoFisher, Catalog number I36007) was used to compare ROS generation between treatment groups. The kit contains 5-(and-6)-carboxy-2', $7^{\prime}$-dichlorodihydrofluorescein diacetate (carboxy- $\mathrm{H}_{2} \mathrm{DCFDA}$ ), Hoechst 33342, tert-butyl hydroperoxide (TBHP), and dimethylsulfoxide (DMSO). Carboxy$\mathrm{H}_{2}$ DCFDA dissolved in DMSO permeates cells, within which it is cleaved by cellular esterases. Cleaved fluorescein compounds then fluoresce proportionally to the amount of circulating ROS within the cell. Live and dead cells stained green throughout this thesis have therefore generated substantial ROS. TBHP is a known inducer of ROS generation and, when used before carboxy$\mathrm{H}_{2}$ DCFDA staining, serves as a positive control. Hoechst 33342 was used in viability assessment, described below.

Following incubation in treatment conditions, cell media was gently rinsed from positive control wells. TBHP solution was added to positive control wells for 60 minutes, at which time the TBHP solution and media from all other wells were removed. Each well was gently rinsed with PBS and carboxy- $\mathrm{H}_{2}$ DCFDA staining solution was added to each well for 20 minutes. After 20 minutes, Hoechst solution and EthD solution were added to each of the wells for 10 minutes. Images were taken immediately following staining. A detailed protocol for evaluation of ROS generation can be found in Appendix $\mathrm{H}$. 


\subsubsection{Viability Assessment}

Cell viability is a ratio of live cells to total cells and is commonly used to quantify the health of a sample group of cultured cells [40]. Hoechst 33342, a blue nuclear stain also known as bisbenzimide, binds the minor groove of double-stranded DNA, creating a concentrated area of fluorescence within each cell's nucleus. The number of visible blue nuclei can therefore be used to represent the total number of cells present. Ethidium Homodimer 1 (EthD) is a red fluorescent molecule which intercalates double-stranded DNA but cannot penetrate an intact cell membrane. Cell membranes are essential for survival, so concentrated areas of red fluorescence signal the presence of a dead cell. Cell viability can be calculated using the following equation [40].

$$
\text { Viability }(\%)=\frac{\text { Hoechst }^{+} \text {Cells }- \text { EthD }{ }^{+} \text {Cells }}{\text { Hoechst }^{+} \text {Cells }} \times 100
$$

Measurements of cell viability were taken from the same samples evaluated for ROS generation which were stained using the combined procedure described above. A detailed protocol for assessment of cell viability can be found in Appendix I.

\subsubsection{Image Processing and Analysis}

Cell counting and fluorescence intensity measurements were conducted in ImageJ [87]. Exported Multi-TIFFs were separated by channel into single-color stacks before being Zprojected to create three images, each with all of the cells that stained positively in that color channel. Images were then thresholded to black and white using the "Default" threshold setting, and the automatic particle analyzer counted all particles greater than $0.001 \mathrm{~cm}^{2}$. This method was developed to maximize signal-to-noise ratio and to minimize measurement bias by eliminating subjective decision-making. Masks were made from thresholded images to facilitate measurement of mean gray value within carboxyl $\mathrm{H}_{2}$-DCFDA-positive areas. Mean gray values 
were normalized to measured area of masks. Detailed protocols for confocal imaging including macros for image processing can be found in Appendix K.

\subsubsection{Statistical Analysis}

Analysis for statistical significance was conducted using JMP [72]. Analysis of variance (ANOVA) compared mean cell viability and mean carboxy-H2DCFDA intensity of stained areas over treatment groups (Basal, Moderate, or High glucose) and treatment lengths (24 or 48 hours). Post-hoc Tukey-Kramer HSD tests were performed to determine differences between groups. Raw data from statistical analysis is available in Appendix M.

\subsection{Results}

The first experiment compared cell viability and ROS generation across 24- and 48-hour treatment durations for HUVECs in Basal and elevated glucose (Moderate and High) media. Independently, the effects of treatment and duration had no effect on cell viability (both $p>0.1$ ). A trend toward significant interaction effects of treatment and duration was noted $(\mathrm{p}=0.056)$. Figure 4.2 shows cell viability (live cell count/total cell count) as a function of treatment (Basal, $5.5 \mathrm{mM}$; Moderate, $10.5 \mathrm{mM}$; High, $25.5 \mathrm{mM}$ ) and duration ( 24 or 48 hours). Tukey's test found no differences between groups. 


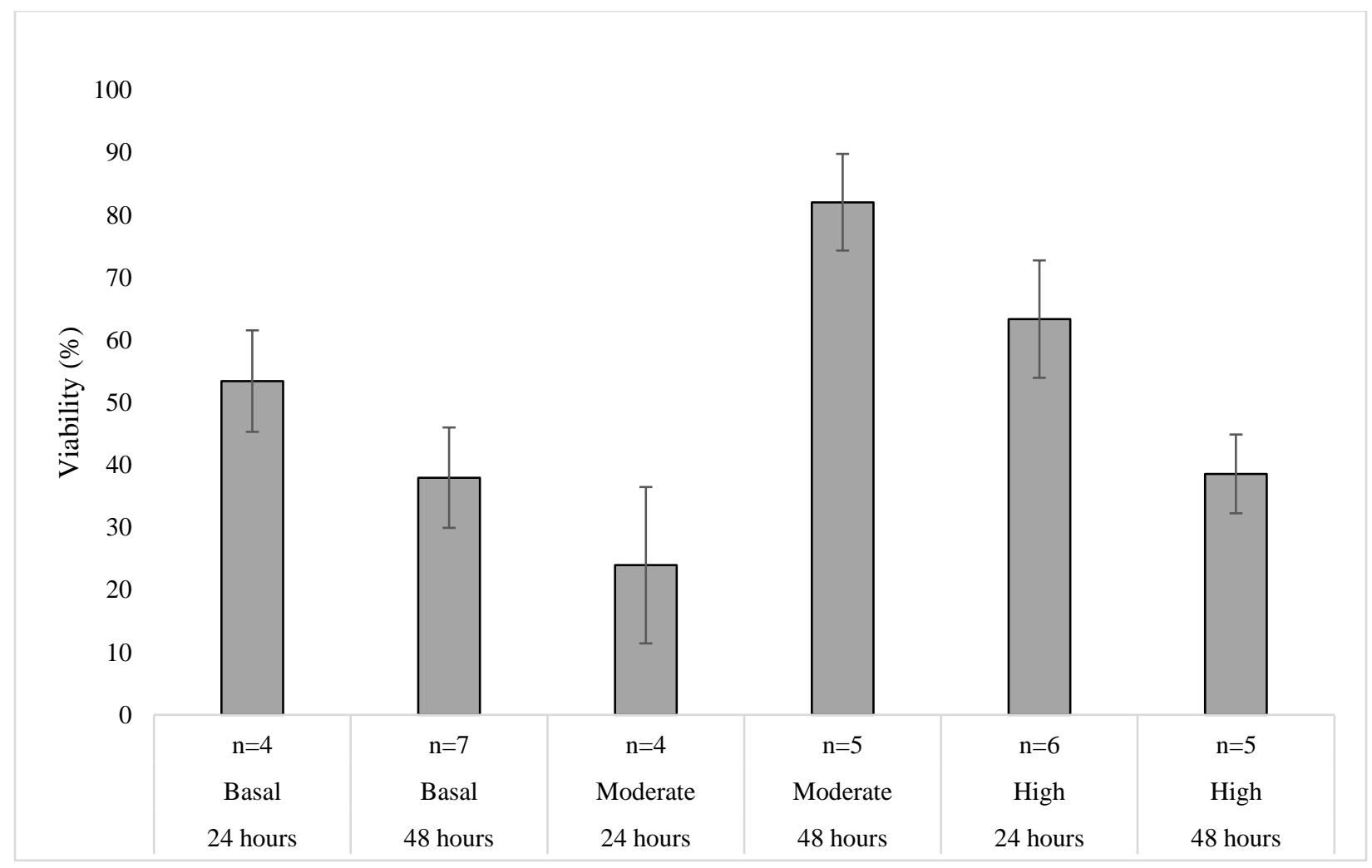

Figure 4.2: Viability by Treatment and Duration. In Basal (5.5 mM) glucose condition, viability increased between 24 and 48 hours. Neither Moderate (10.5 mM) nor High (25.5 mM) glucose condition displayed change in viability between 24 and 48 hours. 
This experiment produced images that were largely oversaturated because laser power was too high. Oversaturation is evident in the EthD channel of these images, recognizable by the uniform color intensity across all stained areas of Figure 4.3C.

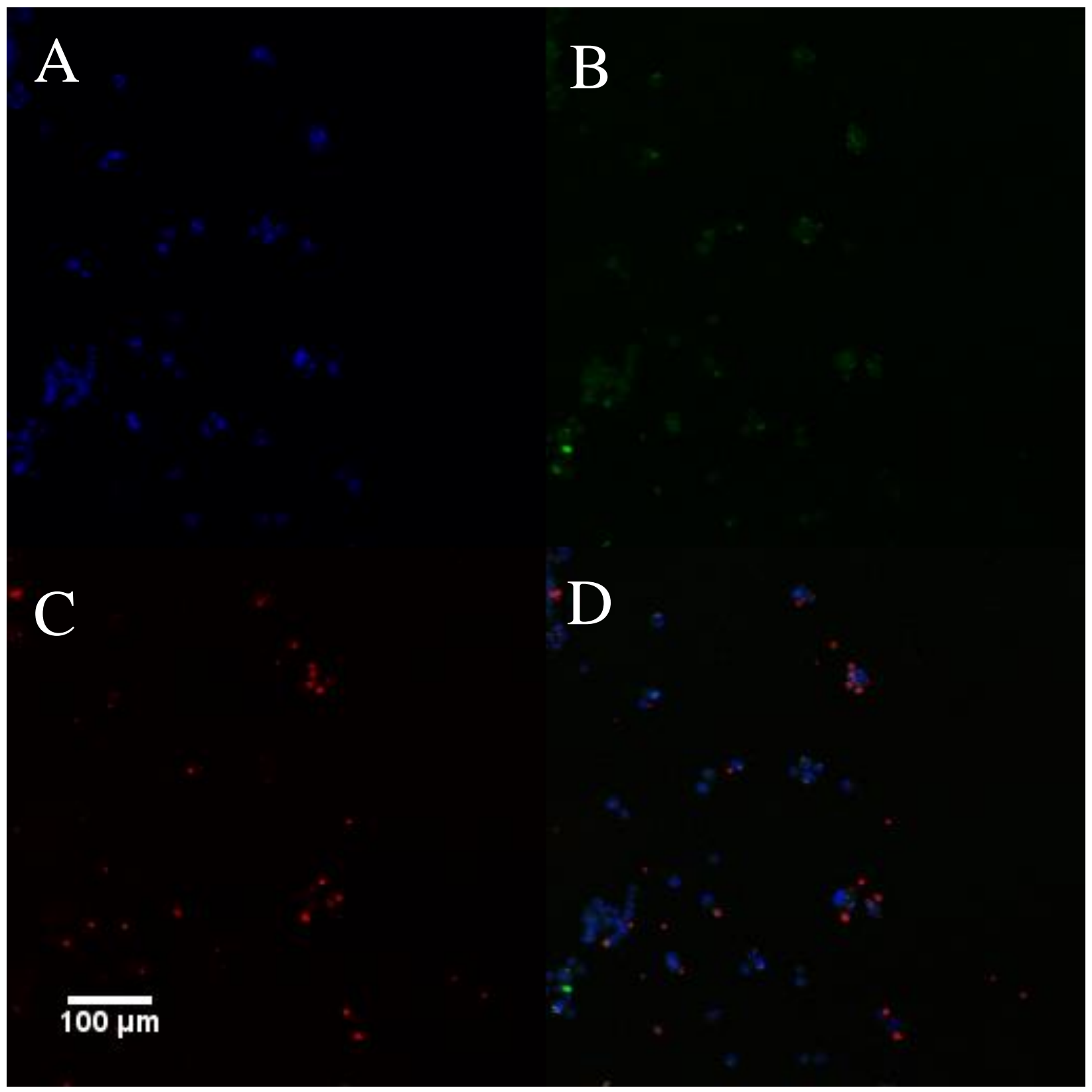

Figure 4.3: Representative Images from Experiment 1. (A) Hoechst 33342-positive (B) CarboxyH2DCFDA-positive (C) EthD-1-positive and (D) Composite images of basal (5.5 mM glucose) treatment condition after 48 hours. 
ROS generation analysis showed no change in mean gray value of stained regions using Tukey’s HSD test. No significant effects of treatment, duration, or interaction effects were detected. ROS evaluation by mean gray value is presented in Figure 4.4 with Positive Control indicating wells which received TBHP treatment, a known inducer of ROS [88].

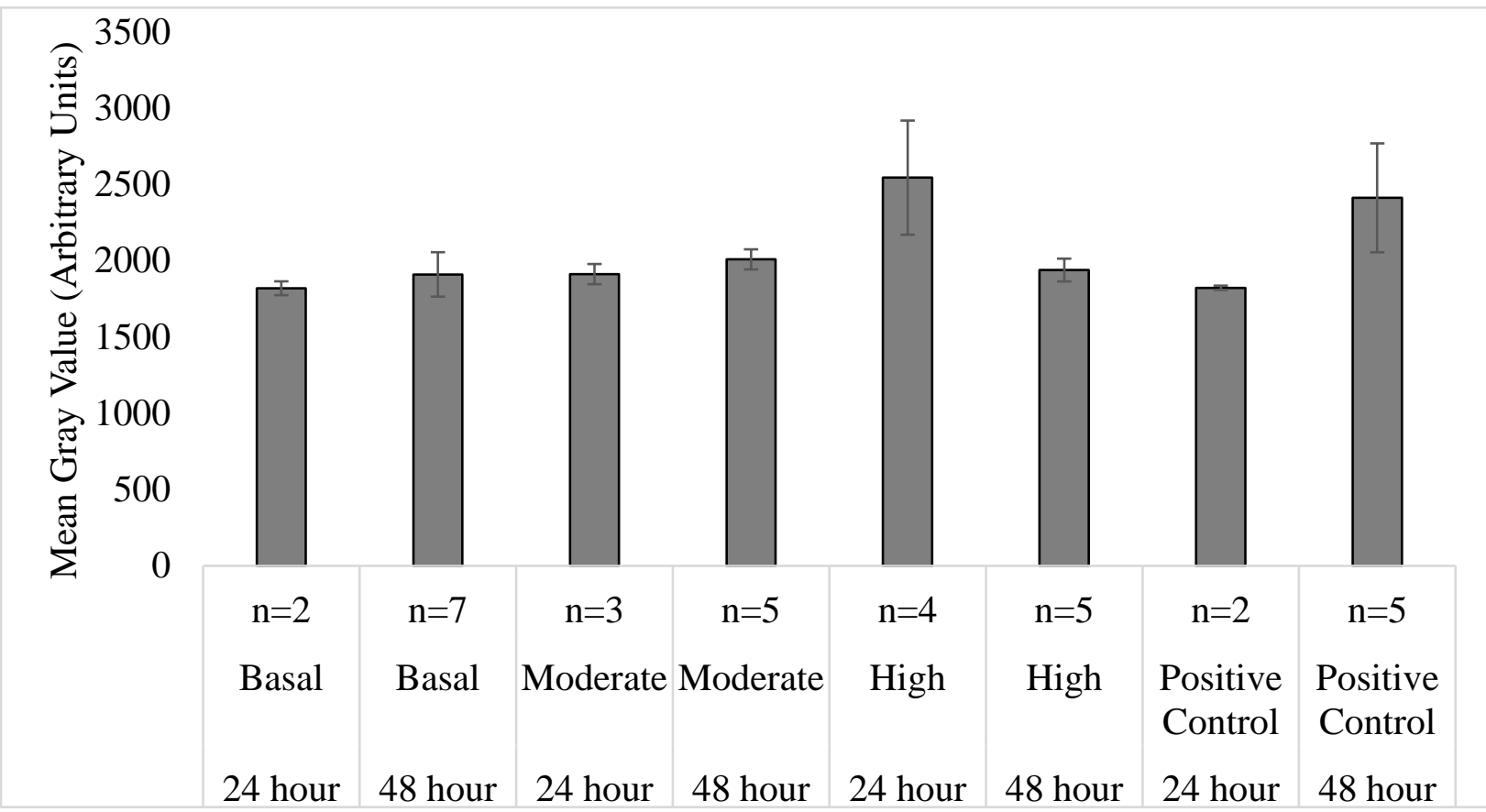

Figure 4.4: ROS Generation as Mean Gray Value by Treatment and Duration. Samples were treated with Basal (5.5 mM), Moderate (10.5 mM), or High (25.5 mM) glucose. Positive Control samples received Basal glucose treatment and TBHP treatment for ROS generation.

Following inconclusive results of Experiment 1, adjustments were made to increase Hoechst concentration during staining and to optimize laser power settings. Experiment 2 also eliminated the 24-hour timepoint to increase sample size and potentially decrease variability. This decision also eliminated the possibility of interaction effects between treatment and duration. 
No statistical effect of treatment on cell viability was detected $(p>0.1)$ in Experiment 2 .

Tukey's HSD test found no differences between groups, including Positive Control samples.

Figure 4.5 displays cell viability by treatment following 48 hours of incubation.

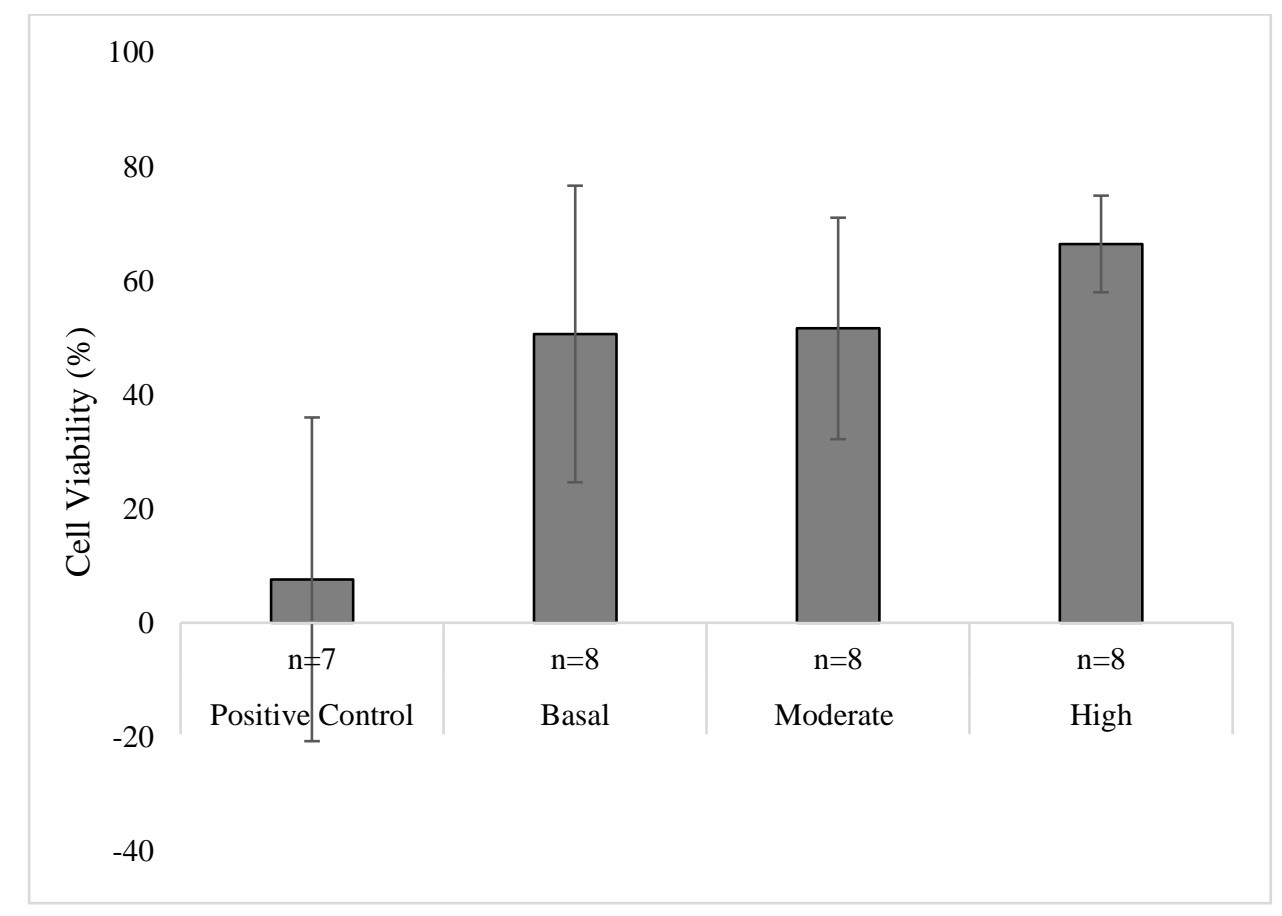

Figure 4.5: Cell Viability by Treatment at 48 Hours. Positive control samples were cultured with basal (5.5 mM) glucose before TBHP treatment which may decrease cell viability. Basal (5.5 mM), Moderate (10.5 mM) and High (25.5 mM) glucose treatments show no effect on cell viability. 
Figure 4.6 displays representative images for Experiment 2. Images from Experiment 2 did not display the same degree of oversaturation as those from Experiment 1, signifying appropriate imaging settings.

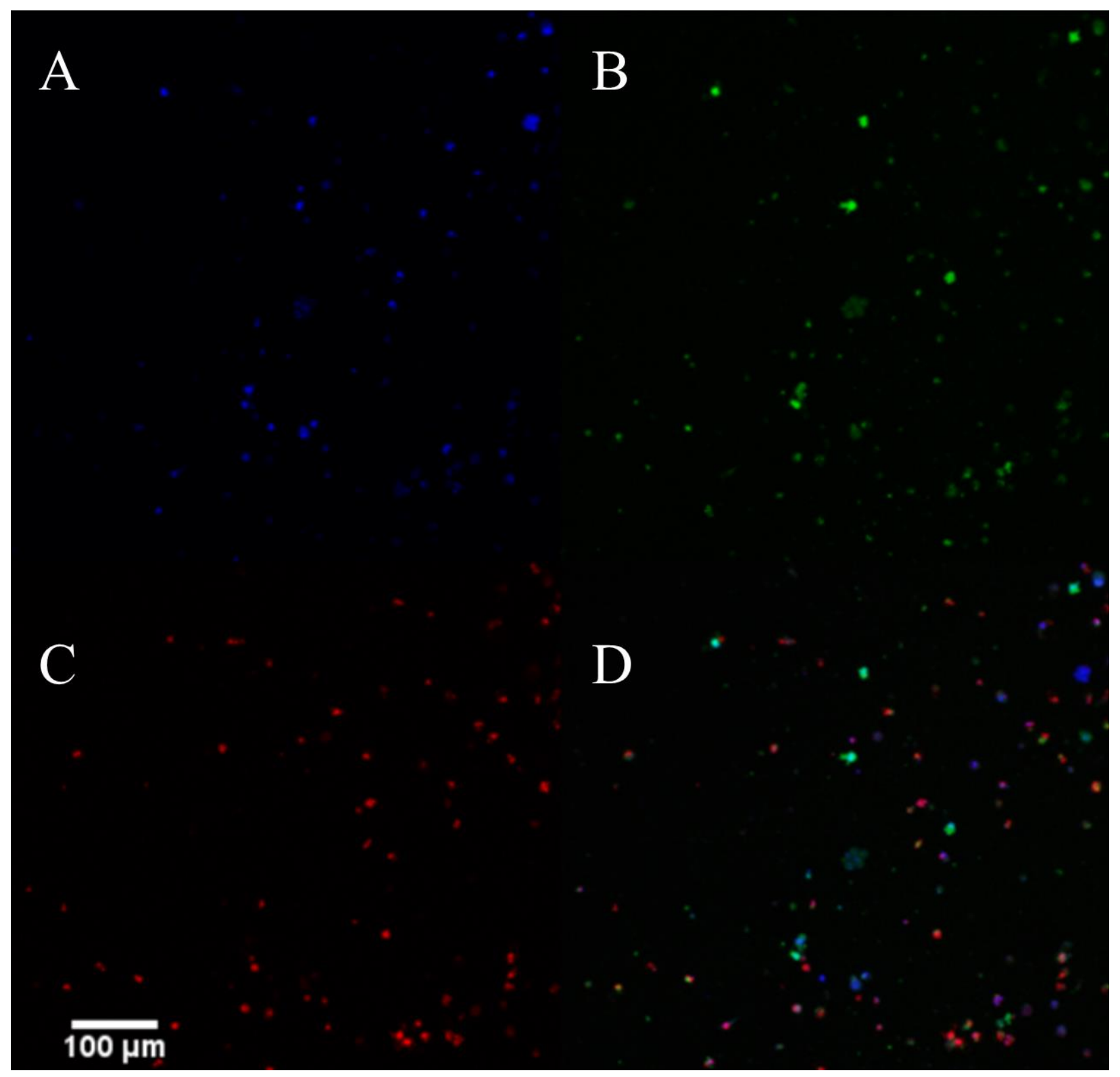

Figure 4.6: Representative Images from Experiment 2. (A) Hoechst 33342-positive (B) CarboxyH2DCFDA-positive (C) EthD-1-positive and (D) Composite images of basal (5.5 mM glucose) treatment condition after 48 hours. 
ROS generation was compared across basal and elevated glucose conditions. These results are separated by imaging day because staining duration, UV exposure, and ambient temperature fluctuated slightly across days; comparing fluorescence intensity can be rendered meaningless by any of these variables [89].

No differences in ROS generation were detected by Tukey's HSD test across treatment groups after 48 hours of treatment ( $>0.1)$. No differences were detected across experiment days $(\mathrm{p}=0.062)$. Figure 4.7 compares Mean Gray Value of carboxy- $\mathrm{H}_{2} \mathrm{DCFDA}-$ positive areas as measured by macros found in Appendix K.
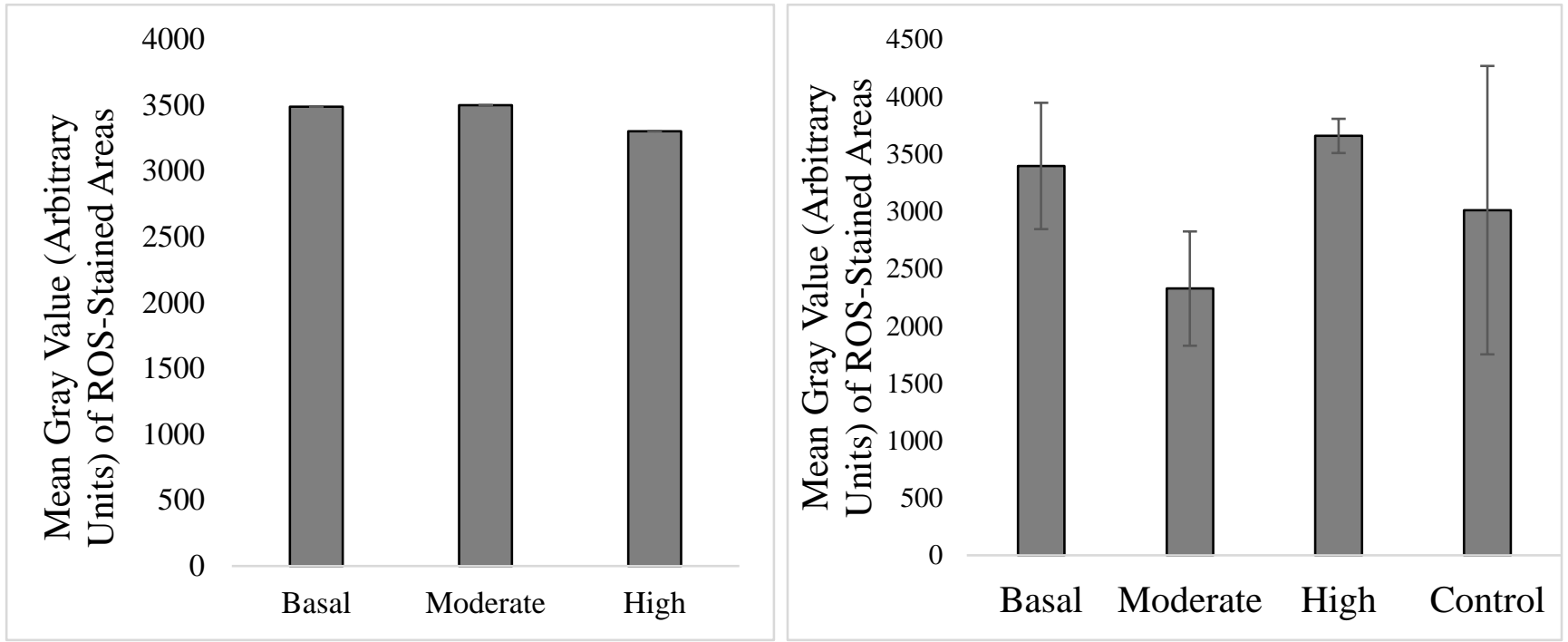

Figure 4.7: ROS Generation on (A) Day 1(n=4 per group) and (B) Day 2 (n=4 per group) of Experiment 2. Samples were treated with Basal (5.5 mM), Moderate (10.5 mM), or High (25.5 mM) glucose. Positive Control samples received Basal glucose treatment and TBHP treatment for ROS generation.

No statistically significant differences define these measurements of ROS generation or cell viability. Differences due to day of assessment and interaction effects were all insignificant. This facilitates combination of all data from this experiment to assess treatment effect. 
Summarized results of each one-way ANOVA are provided in Table 4.4 while complete results are available in Appendix L.

Table 4.4: Summary of Statistical Significance of Imaging Findings

\begin{tabular}{|l|l|l|}
\hline Variable & P-Value & Degrees of Freedom \\
\hline Cell Viability & 0.2965 & 30 \\
\hline $\begin{array}{l}\text { Mean Gray Value of ROS- } \\
\text { Stained Areas }\end{array}$ & 0.6210 & 30 \\
\hline
\end{tabular}

\subsection{Discussion}

Aim 3 was to evaluate Reactive Oxygen Species (ROS) generation at two acute timepoints in elevated glucose conditions by quantifying oxidative stress activity. To this end, ROS generation and cell viability were visualized by fluorescent staining using Hoechst 33342, Ethidium Homodimer-1, and Carboxy-H $\mathrm{H}_{2}$ DCFDA to identify cell nuclei, dead cells, and ROS fragments respectively.

Viability measured the calculated number of live cells $\left(\right.$ Hoechst ${ }^{+}$cells - EthD- $1^{+}$cells) divided by total cells (Hoechst ${ }^{+}$cells) and represents overall well-being [40]. Viability was measured across 24- and 48-hour timepoints at Basal and two high glucose (Moderate and High) culture conditions. Cell viability was consistent across glucose concentrations and treatment durations. Viability decreased in positive control samples treated with TBHP, likely due to its documented cytotoxicity $[54,55]$. TBHP is used to induce ROS generation and can reduce cell viability through DNA damage [7, 8]. In general, these results are inconsistent with previously documented decreases in cell viability $[9,31]$. These results imply that either the previous trend of decreasing cell viability could not be replicated by these methods or that these methods could not detect changes in cell viability. The two-hour duration of the staining protocol may account for this. Due to low staining solution volumes, these solutions rapidly cooled to the ambient 
temperature. While removed from incubation for staining, many cells were seen by eye detaching from culture surfaces, indicative of ongoing cell damage. This observation and the lack of expected changes in cell viability suggest that inconsistency with previous findings is due to a failure of this staining and imaging methodology.

ROS generation was measured by thresholding images to select areas where ROS generation occurred and calculating a mean gray value for those areas. Mean gray value showed no difference across treatment groups, which suggests that either this imaging protocol fails to detect differences in ROS generation or ROS generation is equivalent following 24 or 48 hours of high glucose treatment. A lack of ROS generation under high glucose treatment was inconsistent with mechanism-based predictions. This may also be due to the two-hour duration of the staining protocol. As previously mentioned, staining took place outside of the temperaturecontrolled cell culture environment and staining solutions cooled quickly. Low ambient temperature could directly increase ROS generation, as increased ROS production in response to hypothermia has been documented at $32^{\circ} \mathrm{C}$ [91]. The documented lack of difference in ROS generation between the Basal and Positive Control samples supports this hypothesis. Because both treatments cultured cells in standard media ( $5.5 \mathrm{mM}$ glucose) and the only difference between these groups was treatment with TBHP, which is intended to increase ROS generation, the lack of difference implies that by the time cells are evaluated ROS generation cannot be accurately quantified.

Live imaging protocols like those used to obtain these data rely on the researcher's ability to maintain a physiologic environment throughout staining and imaging. Without dedicated nonsterile incubation equipment, many cells undergo stress and lysis during staining and imaging. These experiments highlight the importance of tailoring protocols to the equipment and resources 
available; a fixation regimen and antibody-mediated fluorescent staining protocol might provide more insight into the oxidative stress pathway. Detection of oxidative stress using immunohistochemistry is often performed by staining for 3-nitrotyrosine, which is a byproduct of ROS-mediated protein modification [92]. A fixated protocol demonstrated increased 3nitrotyrosine staining following 24 hour treatment with $30 \mathrm{mM}$ glucose in BAECs and correlated the increase with increased ROS [93]. Nitrotyrosine was also visualized by IHC in the umbilical cord vessels of women with gestational diabetes [94]. This procedure would include fixation prior to staining, eliminating the need to maintain a physiologic environment during staining and imaging.

In the greater context of this thesis, these data fail to support the high glucose condition as a model for diabetic macrovascular disease. Increased ROS generation with increasing glucose concentration was hypothesized and could have supported upregulation of IL-6 through PKC activation. Cell viability was predicted to decrease with increasing glucose concentration due to glucose-mediated cell damage, however noise was introduced into these measurements by uncontrolled cell damage during staining. It is difficult to determine if the lack of differences in ROS generation and cell viability across treatment groups was due to cellular responses to treatment or was due to thermal stress induced by storage at room temperature during staining and imaging. Investment in non-sterile incubation for use during staining and imaging would resolve this problem, but the cost would be significant. Use of staining protocols which include fixation soon after leaving the sterile culture incubator circumvent this problem. Future work should investigate 3-nitrotyrosine as a target for immunohistochemical staining. Alternatively, the live imaging protocol could be modified to include sterilization of staining materials, which would enable use of cell culture incubators during staining. Either modification would decrease 
problems with the current live imaging protocols and better represent oxidative stress activity in cultured cells and blood vessel mimics. 


\section{DISCUSSION AND CONCLUSION}

\subsection{Summary and Aims of this Thesis}

\section{In contribution towards development of a tissue engineered diabetic macrovascular}

\section{diseased blood vessel mimic, this thesis completed three aims.}

In Aim 1, two genes of interest were determined to represent oxidative stress responses found in diabetic macrovascular disease. Following literature review and preliminary trials with custom primers, IL-6 and CD36 were selected for evaluation. Higher quality predesigned primers were purchased for these two genes, although these primers were mistakenly ordered with probequencher reporters. This necessitated measurement of reaction efficiency to accurately compare treatment groups. Also in pursuit of Aim 1, qPCR data analysis methods were compared and reference gene stability was assessed. ACTB was eliminated due to primer-dimer formation and HPRT1 and B2M demonstrated inconsistent expression, implying instability in at least one reference gene. A lack of stable reference genes determined the gene expression analysis method. $\mathrm{E}^{-\Delta \mathrm{C}} \mathrm{T}$ calculations were selected to represent changes in gene expression from basal to elevated glucose conditions. With genes selected and analysis methodology chosen, cells were evaluated for responses to increased glucose.

In Aim 2, HUVECs were evaluated at seven and fourteen days in high glucose conditions. Glucose concentrations of $5.5 \mathrm{mM}, 10.5 \mathrm{mM}$, and $25.5 \mathrm{mM}$ represented healthy, prediabetic, and diabetic conditions respectively. CD36 expression was consistent across treatment groups at both 7 and 14 days while IL-6 expression increased at both time points. Low CD36 expression supported previous work demonstrating a lack of CD36 expression in HUVECs, associated with their macrovascular phenotype [77]. Increased IL-6 mRNA expression suggests activation of the acute oxidative stress response to high glucose. Hyperacute responses 
to elevated glucose were evaluated next via confocal imaging to capture another aspect of the oxidative stress response.

In Aim 3, fluorescent imaging of reactive oxygen species was used to measure ROS generation in HUVECs treated with the same healthy, prediabetic, and diabetic glucose concentrations for 24 or 48 hours. No trends were evident in ROS generation across treatment groups including the positive control treated with a known inducer of ROS. Cell viability decreased with positive control treatment but was unaffected by high glucose treatment. ROS generation was high and viability was low overall, implying cellular damage from sustained exposure to suboptimal environmental conditions, namely temperature, during the lengthy staining procedure. Alternative evaluations for ROS generation are proposed later in this chapter.

Overall, this thesis contributes one method for assessing cellular responses to elevated glucose, one method for assessing endothelial cell phenotypes, and foundational work toward development of an imaging protocol to represent oxidative stress activity. Demonstration of increasing IL-6 expression with high glucose treatment contributes toward modeling of diabetic macrovascular disease in this laboratory. Measuring CD36 expression was determined irrelevant to assessing oxidative stress behavior in HUVECs, though this evaluation could be used in the future to verify alignment with macrovascular endothelial cell characteristics in cultured cells and BVMs. Assessment of ROS generation and cell viability by confocal microscopy was unsuccessful, however insights from these experiments will be used in the future to accomplish the overall goal of visualizing oxidative stress activity.

\subsection{Challenges and Limitations}

Elevated glucose concentrations in cell media were made using an $80 \mathrm{mM}$ sterile glucose solution, which led to a large volume of cell media being replaced by the water-based solution. 
The high-glucose medias therefore had decreased ionic strength and nutrient concentrations compared to unaltered media. This could have induced hypotonicity or starvation responses in treated cells, obscuring effects of increased glucose concentration. Additionally, glucose concentration was not measured during or following culture, so the rate of glucose utilization is unknown. This may have resulted in fluctuating glucose concentrations based on when cell media was changed.

Previous characterizations of the diabetic macrovascular diseased blood vessel mimic model found increased endothelial nitric oxide synthase (eNOS) mRNA expression after 7 days at $10.5 \mathrm{mM}$ and $25.5 \mathrm{mM}$ in cultured HUVECs [28]. eNOS synthesizes nitric oxide (NO), a potent vasodilator, and inhibits thrombus formation on the endothelial surface [95]. Other genes investigated (Platelet-Endothelial Adhesion Cell Molecule (PECAM), Receptor for Advanced Glycation End Products (RAGE)) had no changes in mRNA expression in cultured HUVECs [28]. Increasing eNOS expression was validated by previous work documenting increased eNOS expression after 5 days of treatment at $25 \mathrm{mM}$ [96]. However, attempts to elucidate signaling cascades have proposed that elevated glucose inhibits eNOS expression [95] and contradictory previous work documents no change in HUVEC eNOS expression after 24 hours [97] and 96 hours [98] of high glucose treatment. This inconsistency in documented findings suggests that IL-6 may be a more meaningful indicator of oxidative stress activity than eNOS.

The unintentional ordering and subsequent use of primers with probe detectors reduced reaction efficiency substantially. This required use of $\mathrm{E}^{-\mathrm{AC}}{ }_{\mathrm{T}}$ measurements rather than idealized 2$\Delta \mathrm{C}_{\mathrm{T}}$ measurements. These comparisons remain valid, however assessments using primers with perfect efficiency may demonstrate more change in mRNA expression across treatment groups. Two reactions using the same starting cDNA but different reaction efficiencies would reach the 
amplification threshold at different times - the high efficiency reaction would have a lower $\mathrm{C}_{\mathrm{T}}$ value and subsequently higher relative mRNA expression than the low efficiency reaction. qPCR using low efficiency may therefore underestimate the degree of change in mRNA expression due to high glucose treatment. The avoidance of probe-quencher primers has already been implemented in laboratory protocols (See Appendix B). Statements of trends throughout this thesis are based on $\mathrm{E}^{-\Delta \mathrm{C}} \mathrm{T}$ measurements, accounting for the mistake in primer ordering. Attempts to verify results with probe detection were unsuccessful, most likely due to repeated exposure to light over the lifespan of the primers leading to extremely high variability and poor performance of the fluorescent markers. Had the fluorescent probes been identified immediately, storage would have included protection from light.

Evaluation of cellular responses to elevated glucose took place at seven and 14 days for qPCR. Levels of mRNA expression at seven and fourteen days cannot be compared directly due to lack of a stable reference gene, however similar trends of increased B2M and IL-6 expression at $25.5 \mathrm{mM}$ glucose were present at both timepoints. This is inconsistent with previous research which found increases in oxidative stress and PKC activity at 7 days of high glucose treatment and further increased expression at 14 days [73]. Seven and 14 days were selected for analysis to facilitate comparison to previous work in this laboratory [28] as well as documented findings $[86,87]$. Many attempts were made to investigate gene expression at 3 days, which would better represent acute responses to oxidative stress. Due to repeated experimental error, none of these samples had adequate RNA purity for assessment by qPCR. This loss of data eliminates comparisons to studies using this treatment duration [88-90] and prevents insight into how early gene expression may change in response to elevated glucose. 
Evaluation of ROS generation and cellular viability were conducted first at 24 and 48 hours and subsequently only at 48 hours. These timepoints correlate well with studies that link ROS overproduction with decreased cell viability or proliferation $[65,79,85]$. To better capture acute ROS generation responses due to high glucose conditions, shorter time points between four and 24 hours should have been investigated [64, 91, 92]. Regardless of treatment duration, this evaluation method failed to capture differences in ROS generation and cell viability across glucose treatment concentrations. As mentioned in chapter 4 , it is impossible to determine if these results accurately represent cell behavior due to damage which occurred during staining and imaging. Due to a lack of dedicated incubation equipment for cells undergoing staining, samples were surrounded by ambient air for at least two hours before being imaged. This led to rapid cooling of cell culture media and staining solutions which increased ROS generation and cell death in all samples due to hypothermia [84, 97]. Rather than investing in cost-prohibitive incubation equipment, fixating samples immediately following high glucose treatments and using an antibody-based stain could be used to identify oxidative stress and ROS production. 3nitrotyrosine a byproduct of ROS protein modifications and is commonly used as a marker for oxidative stress $[98,99]$. Cells in culture or BVMs could be stained for oxidative stress using this method in the future.

This thesis is also limited by the lack of BVM data. Previous investigations in this laboratory found differential mRNA expression between HUVECs treated in culture and HUVECs incorporated into a BVM [28]. As previously mentioned, eNOS was upregulated by high glucose treatment in HUVECs in culture, however eNOS expression was consistent across treatment groups in HUVEC BVMs. Additionally, RAGE expression was consistent across treatment groups in cultured HUVECs but was upregulated by elevated glucose in HUVEC 
BVMs [28]. This emphasizes the role of 3D morphology and shear conditions on HUVEC gene expression and limits the relevance of conclusions made from cells in static 2D culture. Future work related to this will be addressed below.

\subsection{Future Work}

This thesis documents sound methods for qPCR data analysis when reaction efficiency is suboptimal and all of the missteps that led to suboptimal efficiency. Therefore, it would serve the development of a diabetic macrovascular disease model to repeat some of these experiments using primers with ideal reaction efficiency. Notably, IL-6 mRNA expression should continue to be evaluated in future experiments as it represented increased oxidative stress activity due to high glucose treatment. In addition, introduction of an intermittent glucose treatment, where cells are treated with Basal and High glucose medias on alternating days, could further increase oxidative stress and IL-6 activity by more closely replicating acute glucose challenges in Type 2 Diabetic patients $[96,97]$. This research is based on the understanding that acute post-prandial hyperglycemia contributes to the pathogenesis of diabetic complications, though the mechanism for this association is unknown [109]. Previous work, which found increased IL-6 mRNA expression at 7 and 14 days of treatment with $20 \mathrm{mM}$ glucose, found substantially increased expression following intermittent glucose treatment [79]. Replicating the oxidative stress response could also be enhanced by addition of tumor necrosis factor alpha (TNF $\alpha$ ) to endothelial cell media $[99,100]$. TNF $\alpha$ alone and in combination with elevated glucose upregulates ERK1/2 signaling, indicative of diabetic macrovascular disease similarity [102, 104, 105]. Inclusion of intermittent glucose treatments or TNF $\alpha$ in culture media for the diabetic macrovascular disease model could further progress the model toward a diseased state. 
High glucose medias were made using $80 \mathrm{mM}$ glucose in water, which diluted culture media. In the future, stock glucose solution should be made at a $2 \mathrm{M}$ concentration and sterilized via autoclave to reduce the volume of glucose solution required in treatment medias. This will reduce the potential effects of hypotonicity and starvation from media dilution. Additionally, an osmotic control should be added to further isolate the effects of glucose concentration. Mannitol is a molecule which is structurally similar to glucose but is not bioactive. It is commonly used in studies of high glucose in cultured cells [74, 113]. Mannitol stock solutions should also be made at a $2 \mathrm{M}$ concentration and sterilized via autoclave to avoid media dilution.

Rate of glucose consumption in these cells is unknown and should be characterized before investigations of intermittent glucose treatments begin. In order to determine how often cell media should be changed to maintain consistent glucose concentrations, a colorimetric assay (Glucose Assay Kit, Abcam ab65333) should be used to measure glucose concentration in treated cells incrementally until a significant decrease occurs. Cell media should then be changed within the duration of consistent concentration for non-intermittent treatments.

Sodding cells into electrospun scaffolds to create true diabetic macrovascular disease BVM models has been shown in this laboratory to change trends in gene expression due to elevated glucose [28]. This may be due to an effect of peristaltic flow on cellular responses. For this reason, full characterization of HUVEC BVM responses to elevated glucose and intermittent glucose treatments or media supplementation should take place alongside assessments of cells in 2D culture. Following the characterization of HUVECs in culture and in BVMs, similarity to the disease state in vivo could be increased by evaluation of Diabetic Human Coronary Artery Endothelial Cells (DHCAECs) for responses to the same treatments. DHCAECs have been shown in this laboratory to exhibit differential responses to high glucose treatment in 2D culture 
and in BVMs compared to HUVECs in 2D culture and in BVMs [28]. Another potential modification to increase similarity to the cell environment in vivo is the inclusion of smooth muscle cells sodded onto the scaffold before endothelial cell sodding. While dual-sodded vessels have been investigated previously for other applications, methods for qPCR on dual-sodded vessels have not been developed.

Assessing oxidative stress by confocal imaging facilitates powerful visualizations of cell responses. Without stage-mounted incubators and a dedicated non-sterile incubator, cells undergo oxidative stress during the staining and imaging procedures. Improvement of live imaging protocols to better suit available equipment could reduce thermal stress experienced by samples. Staining solutions are sterile upon arrival, so conducting all staining inside the cell culture hood would allow samples to be returned to the cell culture incubator while stains penetrate live cells. Previous development of a low-cost microscope stage heater by Vahid Hamzeinejad under the guidance of Dr. Trevor Cardinal could facilitate maintaining physiologic temperature during imaging. Utilization of stage heaters is essential in Animal Science laboratories for the investigation of embryos, so commercial equipment could potentially be borrowed from that department in the future. Either of these options would substantially improve the data collected from live imaging.

Alternative methods like immunohistochemical staining for 3-nitrotyrosine allow fixation, preventing cell damage before analysis. HUVECs, DHCAECs, and BVMs made from both cell types can all be fixated in formalin before incubation with an anti-3-nitrotyrosine primary antibody (e.g. Abcam, ab61932), blocking, and incubating with a complementary secondary antibody conjugated to a fluorescent molecule [92]. Measuring stained areas in 2D cultured samples and BVMs could quantify severity of oxidative stress [106]. This imaging 
modality is better suited to equipment and resources available to this laboratory and should be investigated alongside development of the diabetic macrovascular disease BVM model.

\subsection{Conclusion}

This thesis supports the method of elevated extracellular glucose as contribution toward an oxidative stress state in HUVECs as measured by qPCR. IL-6 expression increased, consistent with diabetic macrovascular disease pathology [6-8]. CD36 expression remained consistently low across treatment groups, which was unanticipated but validated by a documented lack of CD36 expression in HUVECs [81,90].

This thesis rejects live cell imaging, as performed, for measuring ROS generation as a feasible method of determining oxidative stress activity in this model. Improvement of temperature regulation during staining and imaging would increase data relevance. Additionally, staining protocols that allow fixation should be investigated as a means to detect ROS generation in HUVECs; these protocols can be applied both in culture and in BVMs using ROS levels as an indicator of early oxidative stress responses.

Cell viability also failed to decrease in response to elevated glucose as has been documented [44]. This could also be a result of the extended live cell evaluation. Future work to characterize the full diabetic macrovascular disease BVM model including evaluation of diabetic cells and incorporation into 3D scaffolds will progress the model toward relevant conclusions about vascular devices. Additional simulation of the disease state by inducing intermittent glucose treatments or incorporating TNF $\alpha$ into culture media will accentuate relevance of the model. Imaging protocols utilizing fixatives will minimize the effects of staining duration on quantitative image analysis. 
In summary, this thesis contributes thorough comparisons of data analysis methods for qPCR and preliminary evaluation of changes in gene expression in response to sustained elevated glucose conditions. Insight into the relevance of live imaging protocols to this project were also provided and an alternative method was proposed. This thesis serves as a launching point for further characterization of the diabetic macrovascular disease BVM model. 


\section{REFERENCES}

[1] Centers for Disease Control, "National Diabetes Statistics Report," 2017. [Online]. Available: https://www.cdc.gov/diabetes/data/statistics-report/index.html. [Accessed: 14Apr-2018].

[2] G. N. Ioannou, C. L. Bryson, and E. J. Boyko, "Prevalence and trends of insulin resistance, impaired fasting glucose, and diabetes," J. Diabetes Complications, vol. 21, no. 6, pp. 363-370, Nov. 2007.

[3] S. D. Funk, A. Yurdagul, A. W. Orr, and A. W. Orr, "Hyperglycemia and endothelial dysfunction in atherosclerosis: lessons from type 1 diabetes.," Int. J. Vasc. Med., vol. 2012, p. 569654, 2012.

[4] P. Ebeling, H. A. Koistinen, and V. A. Koivisto, "Insulin-independent glucose transport regulates insulin sensitivity," FEBS Lett., vol. 436, no. 3, pp. 301-303, Oct. 1998.

[5] R. Maughan, "Carbohydrate metabolism,” 2013.

[6] A. D’Souza, M. Hussain, F. C. Howarth, N. M. Woods, K. Bidasee, and J. Singh, "Pathogenesis and pathophysiology of accelerated atherosclerosis in the diabetic heart," Mol. Cell. Biochem., vol. 331, no. 1-2, pp. 89-116, Nov. 2009.

[7] L. Feng, C. Matsumoto, A. Schwartz, A. M. Schmidt, D. M. Stern, and J. Pile-Spellman, "Chronic vascular inflammation in patients with type 2 diabetes: endothelial biopsy and RT-PCR analysis.," Diabetes Care, vol. 28, no. 2, pp. 379-84, Feb. 2005.

[8] H. A. R. Hadi and J. Al Suwaidi, "Endothelial dysfunction in diabetes mellitus.," Vasc. Health Risk Manag., vol. 3, no. 6, pp. 853-76, 2007.

[9] G. Basta, "Receptor for advanced glycation endproducts and atherosclerosis: From basic mechanisms to clinical implications," Atherosclerosis, vol. 196, no. 1, pp. 9-21, Jan. 
2008.

[10] M. KANEKO et al., "Aldose Reductase and AGE-RAGE Pathways: Key Players in Myocardial Ischemic Injury,” Ann. N. Y. Acad. Sci., vol. 1043, no. 1, pp. 702-709, Jun. 2005.

[11] E. R. Duncan et al., "Effect of endothelium-specific insulin resistance on endothelial function in vivo.," Diabetes, vol. 57, no. 12, pp. 3307-14, Dec. 2008.

[12] T. Kubota et al., "Impaired insulin signaling in endothelial cells reduces insulin-induced glucose uptake by skeletal muscle.," Cell Metab., vol. 13, no. 3, pp. 294-307, Mar. 2011.

[13] M. C. Gage et al., "Endothelium-specific insulin resistance leads to accelerated atherosclerosis in areas with disturbed flow patterns: A role for reactive oxygen species," Atherosclerosis, vol. 230, no. 1, pp. 131-139, Sep. 2013.

[14] S. K. Srivastava, K. V. Ramana, and A. Bhatnagar, "Role of Aldose Reductase and Oxidative Damage in Diabetes and the Consequent Potential for Therapeutic Options," Endocr. Rev., vol. 26, no. 3, pp. 380-392, May 2005.

[15] A. Habib et al., "Everolimus-eluting stents improve vascular response in a diabetic animal model.," Circ. Cardiovasc. Interv., vol. 7, no. 4, pp. 526-32, Aug. 2014.

[16] A. Loos, R. Rohde, A. Haverich, and S. Barlach, "In Vitro and In Vivo Biocompatibility Testing of Absorbable Metal Stents," Macromol. Symp., vol. 253, no. 1, pp. 103-108, Aug. 2007.

[17] S. Elezi et al., "Diabetes mellitus and the clinical and angiographic outcome after coronary stent placement," J. Am. Coll. Cardiol., vol. 32, no. 7, pp. 1866-1873, Dec. 1998.

[18] J. P. Carrozza, R. E. Kuntz, R. F. Fishman, and D. S. Baim, "Restenosis after arterial 
injury caused by coronary stenting in patients with diabetes mellitus.," Ann. Intern. Med., vol. 118, no. 5, pp. 344-9, Mar. 1993.

[19] E. Van Belle, K. Abolmaali, C. Bauters, E. P. McFadden, J.-M. Lablanche, and M. E. Bertrand, "Restenosis, late vessel occlusion and left ventricular function six months after balloon angioplasty in diabetic patients," J. Am. Coll. Cardiol., vol. 34, no. 2, pp. 476485, Aug. 1999.

[20] V. Mathew, "Outcomes in Patients With Diabetes Mellitus Undergoing Percutaneous Coronary Intervention in the Current Era: A Report From the Prevention of REStenosis with Tranilast and its Outcomes (PRESTO) Trial," Circulation, vol. 109, no. 4, pp. 476480, Jan. 2004.

[21] D. Carrié, "The Use of the Cre8 Stent in Patients with Diabetes Mellitus," Interv. Cardiol. Rev., vol. 11, no. 1, p. 47, 2016.

[22] L. E. Leigh Perkins, "Preclinical Models of Restenosis and Their Application in the Evaluation of Drug-Eluting Stent Systems," Vet. Pathol., vol. 47, no. 1, pp. 58-76, Jan. 2010.

[23] A. Lafont and D. Faxon, "Why do animal models of post-angioplasty restenosis sometimes poorly predict the outcome of clinical trials?," Cardiovasc. Res., vol. 39, no. 1, pp. 50-59, Jul. 1998.

[24] Y. Naka et al., "RAGE Axis Animal Models and Novel Insights Into the Vascular Complications of Diabetes."

[25] W. T. Cefalu, "Animal Models of Type 2 Diabetes: Clinical Presentation and Pathophysiological Relevance to the Human Condition," ILAR J., vol. 47, no. 3, pp. 186198, Jan. 2006. 
[26] M. C. Gibbons, M. A. Foley, and K. O. Cardinal, “Thinking inside the box: keeping tissue-engineered constructs in vitro for use as preclinical models.," Tissue Eng. Part B. Rev., vol. 19, no. 1, pp. 14-30, Feb. 2013.

[27] S. Herting et al., "Human Umbilical Versus Coronary Cell Sources for Tissue-Engineered Blood Vessel Mimics,” Appl. Vitr. Toxicol., vol. 2, no. 3, pp. 175-182, Sep. 2016.

[28] Shelby Kunz, "Development and Characterization of Tissue Engineered Blood Vessel Mimics Under 'Diabetic' Conditions,” Cal Poly, San Luis Obispo, 2017.

[29] M. G. Vander Heiden, L. C. Cantley, and C. B. Thompson, "Understanding the Warburg effect: the metabolic requirements of cell proliferation.," Science, vol. 324, no. 5930, pp. 1029-33, May 2009.

[30] B. C. Mulukutla, S. Khan, A. Lange, and W.-S. Hu, "Glucose metabolism in mammalian cell culture: new insights for tweaking vintage pathways," Trends Biotechnol., vol. 28, no. 9, pp. 476-484, Sep. 2010.

[31] H. Farhangkhoee, Z. A. Khan, Y. Barbin, and S. Chakrabarti, "Glucose-induced upregulation of CD36 mediates oxidative stress and microvascular endothelial cell dysfunction," Diabetologia, vol. 48, no. 7, pp. 1401-1410, Jul. 2005.

[32] L. E. WOLD, A. F. CEYLAN-ISIK, and J. REN, "Oxidative stress and stress signaling: menace of diabetic cardiomyopathy," Acta Pharmacol. Sin., vol. 26, no. 8, pp. 908-917, Aug. 2005.

[33] T. Fiorentino, A. Prioletta, P. Zuo, and F. Folli, "Hyperglycemia-induced Oxidative Stress and its Role in Diabetes Mellitus Related Cardiovascular Diseases," Curr. Pharm. Des., vol. 19, no. 32, pp. 5695-5703, Aug. 2013.

[34] K. Boulay et al., "The IL-6-Soluble IL-6R $\square$ Autocrine Loop of Endothelial Activation as 
an Intermediate Between Acute and Chronic Inflammation: an Experimental Model Involving Thrombin,” J Immunol Ref. J. Immunol., vol. 167, pp. 3435-3442, 2018.

[35] D. Aronson and E. J. Rayfield, "How hyperglycemia promotes atherosclerosis: molecular mechanisms," Cardiovasc. Diabetol., vol. 1, no. 1, p. 1, Apr. 2002.

[36] P. J. Boyle, "Diabetes Mellitus and Macrovascular Disease: Mechanisms and Mediators," Am. J. Med., vol. 120, no. 9, pp. S12-S17, Sep. 2007.

[37] S. Bakhashab et al., "Reference genes for expression studies in hypoxia and hyperglycemia models in human umbilical vein endothelial cells.," G3 (Bethesda)., vol. 4, no. 11, pp. 2159-65, Sep. 2014.

[38] V. S. Baddela, A. Baufeld, V. R. Yenuganti, J. Vanselow, and D. Singh, "Suitable housekeeping genes for normalization of transcript abundance analysis by real-time RTPCR in cultured bovine granulosa cells during hypoxia and differential cell plating density.," Reprod. Biol. Endocrinol., vol. 12, p. 118, Nov. 2014.

[39] N. Silver, S. Best, J. Jiang, and S. Thein, "Selection of housekeeping genes for gene expression studies in human reticulocytes using real-time PCR," BMC Mol. Biol., vol. 7, no. 1 , p. 33, Oct. 2006.

[40] T. L. Riss et al., Cell Viability Assays. Eli Lilly \& Company and the National Center for Advancing Translational Sciences, 2004.

[41] X. L. Du et al., "Hyperglycemia-induced mitochondrial superoxide overproduction activates the hexosamine pathway and induces plasminogen activator inhibitor-1 expression by increasing Sp1 glycosylation.," Proc. Natl. Acad. Sci. U. S. A., vol. 97, no. 22, pp. 12222-6, Oct. 2000.

[42] E. Deniaud, J. Baguet, A.-L. Mathieu, G. Pagès, J. Marvel, and Y. Leverrier, 
"Overexpression of Sp1 transcription factor induces apoptosis," Oncogene, vol. 25, no. 53, pp. 7096-7105, Nov. 2006.

[43] M. Brownlee, "The pathobiology of diabetic complications: a unifying mechanism.," Diabetes, vol. 54, no. 6, pp. 1615-25, Jun. 2005.

[44] T. J. LaRocca, "Programmed cell death dynamics during hyperglycemia and ischemic brain injuries.," J. Neurol. Neurorehabilitation Res., vol. 1, no. 2.

[45] J. Rani et al., "T2DiACoD: A Gene Atlas of Type 2 Diabetes Mellitus Associated Complex Disorders," Sci. Rep., vol. 7, no. 1, p. 6892, Dec. 2017.

[46] G. B. Di Gregorio et al., "Expression of CD68 and macrophage chemoattractant protein-1 genes in human adipose and muscle tissues: association with cytokine expression, insulin resistance, and reduction by pioglitazone.," Diabetes, vol. 54, no. 8, pp. 2305-13, Aug. 2005.

[47] M. Banerjee and M. Saxena, "Interleukin-1 (IL-1) family of cytokines: Role in Type 2 Diabetes," Clin. Chim. Acta, vol. 413, no. 15-16, pp. 1163-1170, Aug. 2012.

[48] O. P. Kristiansen and T. Mandrup-Poulsen, "Interleukin-6 and diabetes: the good, the bad, or the indifferent?," Diabetes, vol. 54 Suppl 2, no. suppl 2, pp. S114-24, Dec. 2005.

[49] L. Jackisch et al., "Differential expression of Lp-PLA2 in obesity and type 2 diabetes and the influence of lipids," Diabetologia, vol. 61, no. 5, pp. 1155-1166, Feb. 2018.

[50] S. J. Persaud, C. J. Burns, V. D. Belin, and P. M. Jones, "Glucose-induced regulation of COX-2 expression in human islets of Langerhans.," Diabetes, vol. 53 Suppl 1, no. suppl 1, pp. S190-2, Feb. 2004.

[51] M. C. LaPointe and J. R. Sitkins, "Phospholipase A2 metabolites regulate inducible nitric oxide synthase in myocytes.," Hypertens. (Dallas, Tex. 1979), vol. 31, no. 1 Pt 2, pp. 
218-24, Jan. 1998.

[52] B. J. Morris, A. M. Markus, C. L. Glenn, D. J. Adams, S. Colagiuri, and X. Wang, “Association of a functional inducible nitric oxide synthase promoter variant with complications in type 2 diabetes," J. Mol. Med., vol. 80, no. 2, pp. 96-104, Feb. 2002.

[53] P. R. Nagareddy, Z. Xia, J. H. McNeill, and K. M. MacLeod, "Increased expression of iNOS is associated with endothelial dysfunction and impaired pressor responsiveness in streptozotocin-induced diabetes," Am. J. Physiol. Circ. Physiol., vol. 289, no. 5, pp. H2144-H2152, Nov. 2005.

[54] L. Feng, C. Gu, Y. Li, and J. Huang, "High Glucose Promotes CD36 Expression by Upregulating Peroxisome Proliferator-Activated Receptor $\gamma$ Levels to Exacerbate Lipid Deposition in Renal Tubular Cells," Biomed Res. Int., vol. 2017, pp. 1-10, Apr. 2017.

[55] V. Bermúdez et al., "PPAR- $\gamma$ Agonists and Their Role in Type 2 Diabetes Mellitus Management," Am. J. Ther., vol. 17, no. 3, pp. 274-283, May 2010.

[56] A. C. Frazier-Wood et al., "Genome-wide association study indicates variants associated with insulin signaling and inflammation mediate lipoprotein responses to fenofibrate.," Pharmacogenet. Genomics, vol. 22, no. 10, pp. 750-7, Oct. 2012.

[57] D. J. Kennedy, S. Kuchibhotla, K. M. Westfall, R. L. Silverstein, R. E. Morton, and M. Febbraio, “A CD36-dependent pathway enhances macrophage and adipose tissue inflammation and impairs insulin signalling," Cardiovasc. Res., vol. 89, no. 3, pp. 604613, Feb. 2011.

[58] K. C. B. Tan, W.-S. Chow, S. Tam, R. Bucala, and J. Betteridge, “Association between acute-phase reactants and advanced glycation end products in type 2 diabetes.," Diabetes Care, vol. 27, no. 1, pp. 223-8, Jan. 2004. 
[59] K. J. Livak and T. D. Schmittgen, "Analysis of Relative Gene Expression Data Using Real-Time Quantitative PCR and the 2- $\Delta \Delta$ CT Method," Methods, vol. 25, no. 4, pp. 402408, Dec. 2001.

[60] T. D. Schmittgen and K. J. Livak, "Analyzing real-time PCR data by the comparative CT method," Nat. Protoc., vol. 3, no. 6, pp. 1101-1108, Jun. 2008.

[61] K. J. Livak and T. D. Schmittgen, “Analysis of Relative Gene Expression Data Using Real- Time Quantitative PCR and the $2 x \square \square \mathrm{C}$ T Method," METHODS, vol. 25, pp. 402408, 2001.

[62] J. Vandesompele et al., "Accurate normalization of real-time quantitative RT-PCR data by geometric averaging of multiple internal control genes.," Genome Biol., vol. 3, no. 7, p. RESEARCH0034, Jun. 2002.

[63] J. Vandesompele et al., "Accurate normalization of real-time quantitative RT-PCR data by geometric averaging of multiple internal control genes.," Genome Biol., vol. 3, no. 7, p. RESEARCH0034, Jun. 2002.

[64] M. Keller et al., "Genome-wide DNA promoter methylation and transcriptome analysis in human adipose tissue unravels novel candidate genes for obesity," Mol. Metab., vol. 6, no. 1, pp. 86-100, Jan. 2017.

[65] K. Lind, A. Ståhlberg, N. Zoric, and M. Kubista, "Combining sequence-specific probes and DNA binding dyes in real-time PCR for specific nucleic acid quantification and melting curve analysis.," Biotechniques, vol. 40, no. 3, pp. 315-9, Mar. 2006.

[66] E. S. G. Cheah et al., "A two-tube combined TaqMan/SYBR Green assay to identify mycobacteria and detect single global lineage-defining polymorphisms in Mycobacterium tuberculosis.," J. Mol. Diagn., vol. 12, no. 2, pp. 250-6, Mar. 2010. 
[67] M. Van Poucke, A. Van Zeveren, and L. Peelman, "[Letter to the editor] Combined FAMlabeled TaqMan probe detection and SYBR green I melting curve analysis in multiprobe qPCR genotyping assays,” Biotechniques, vol. 52, no. 2, pp. 81-6, Feb. 2012.

[68] H. Maeda et al., "Quantitative real-time PCR using TaqMan and SYBR Green for Actinobacillus actinomycetemcomitans, Porphyromonas gingivalis, Prevotella intermedia , tetQ gene and total bacteria," FEMS Immunol. Med. Microbiol., vol. 39, no. 1, pp. 81-86, Oct. 2003.

[69] C.-L. Cheung, K. S. L. Lam, and B. M. Y. Cheung, "Serum $\beta-2$ microglobulin predicts mortality in people with diabetes.," Eur. J. Endocrinol., vol. 169, no. 1, pp. 1-7, Jul. 2013.

[70] S. Cikos, A. Bukovská, and J. Koppel, "Relative quantification of mRNA: comparison of methods currently used for real-time PCR data analysis.," BMC Mol. Biol., vol. 8, p. 113, Dec. 2007.

[71] BERSE et al., "Hypoxia augments cytokine (transforming growth factor-beta (TGF-beta) and IL-1)-induced vascular endothelial growth factor secretion by human synovial fibroblasts," Clin. Exp. Immunol., vol. 115, no. 1, pp. 176-182, Jan. 1999.

[72] SAS Institute Inc., “JMP®, Version 12.1.” Cary, NC, 2007.

[73] L. Quagliaro, L. Piconi, R. Assaloni, L. Martinelli, E. Motz, and A. Ceriello, "Intermittent high glucose enhances apoptosis related to oxidative stress in human umbilical vein endothelial cells: the role of protein kinase $\mathrm{C}$ and $\mathrm{NAD}(\mathrm{P}) \mathrm{H}$-oxidase activation.," Diabetes, vol. 52, no. 11, pp. 2795-804, Nov. 2003.

[74] S. Devaraj, S. K. Venugopal, U. Singh, and I. Jialal, "Hyperglycemia induces monocytic release of interleukin-6 via induction of protein kinase c-\{alpha\} and -\{beta\}.," Diabetes, vol. 54, no. 1, pp. 85-91, Jan. 2005. 
[75] Y. C. Hwang, S. Shaw, M. Kaneko, H. Redd, M. B. Marrero, and R. Ramasamy, “Aldose reductase pathway mediates JAK-STAT signaling: a novel axis in myocardial ischemic injury,” FASEB J., vol. 19, no. 7, pp. 795-797, May 2005.

[76] J.-T. Chi et al., "Endothelial cell diversity revealed by global expression profiling," 2003.

[77] R. A. Swerlick, K. H. Lee, T. M. Wick, and T. J. Lawley, "Human dermal microvascular endothelial but not human umbilical vein endothelial cells express CD36 in vivo and in vitro.," J. Immunol., vol. 148, no. 1, pp. 78-83, Jan. 1992.

[78] P. Kumar, K. Natarajan, and N. Shanmugam, "High glucose driven expression of proinflammatory cytokine and chemokine genes in lymphocytes: Molecular mechanisms of IL-17 family gene expression," Cell. Signal., vol. 26, no. 3, pp. 528-539, Mar. 2014.

[79] L. Piconi et al., "Intermittent high glucose enhances ICAM-1, VCAM-1, E-selectin and interleukin-6 expression in human umbilical endothelial cells in culture: the role of poly(ADP-ribose) polymerase," J. Thromb. Haemost., vol. 2, no. 8, pp. 1453-1459, Aug. 2004.

[80] S. Kotla and G. N. Rao, "ROS mediates p300-dependent STAT1 interaction with PPAR $\gamma$ in CD36 expression and foam cell formation."

[81] M. Yano et al., "Short-term exposure of high glucose concentration induces generation of reactive oxygen species in endothelial cells: implication for the oxidative stress associated with postprandial hyperglycemia," Redox Rep., vol. 9, no. 2, pp. 111-116, Apr. 2004.

[82] M. Zanetti, R. Zwacka, J. Engelhardt, Z. Katusic, and T. O’Brien, "Superoxide anions and endothelial cell proliferation in normoglycemia and hyperglycemia.," Arterioscler.

Thromb. Vasc. Biol., vol. 21, no. 2, pp. 195-200, Feb. 2001.

[83] D. M. Katschinski, K. Boos, S. G. Schindler, and J. Fandrey, "Pivotal role of reactive 
oxygen species as intracellular mediators of hyperthermia-induced apoptosis.," J. Biol.

Chem., vol. 275, no. 28, pp. 21094-8, Jul. 2000.

[84] K. Sada et al., "Hyperglycemia Induces Cellular Hypoxia through Production of Mitochondrial ROS Followed by Suppression of Aquaporin-1,” PLoS One, vol. 11, no. 7, p. e0158619, Jul. 2016.

[85] E. J. Smart and X.-A. Li, "Hyperglycemia: Cell death in a cave," Biochim. Biophys. Acta Mol. Basis Dis., vol. 1772, no. 5, pp. 524-526, May 2007.

[86] S. Ghosh et al., "Role of dietary fatty acids and acute hyperglycemia in modulating cardiac cell death," Nutrition, vol. 20, no. 10, pp. 916-923, Oct. 2004.

[87] W. Rasband, "ImageJ," U. S. Natl. Institutes Heal. Bethesda, Maryland, USA, p. //imagej.nih.gov/ij/, 2012.

[88] O. Kučera et al., "The effect of tert-butyl hydroperoxide-induced oxidative stress on lean and steatotic rat hepatocytes in vitro.," Oxid. Med. Cell. Longev., vol. 2014, p. 752506, Mar. 2014.

[89] ThermoFisher, "Generating and Detecting Reactive Oxygen Species-Section 18.2 - US."

[90] N. J. Association for Research in Vision and Ophthalmology. and A. Spector, "Tertiary Butyl Hydroperoxide (tBHP) Induced DNA Damage, DNA Repair and Cell Death in Human Lens Epithelial (HLE) Cell Cultures,” Invest. Ophthalmol. Vis. Sci., vol. 44, no. 13, pp. 307-307, May 2003.

[91] U. Rauen and H. de Groot, "Cold-Induced Release of Reactive Oxygen Species as a Decisive Mediator of Hypothermia Injury to Cultured Liver Cells," Free Radic. Biol. Med., vol. 24, no. 7-8, pp. 1316-1323, May 1998.

[92] G.-Y. Liou and P. Storz, "Detecting reactive oxygen species by immunohistochemistry.," 
Methods Mol. Biol., vol. 1292, pp. 97-104, 2015.

[93] C. Quijano, L. Castro, G. Peluffo, V. Valez, and R. Radi, "Enhanced mitochondrial superoxide in hyperglycemic endothelial cells: direct measurements and formation of hydrogen peroxide and peroxynitrite," Am. J. Physiol. Circ. Physiol., vol. 293, no. 6, pp. H3404-H3414, Dec. 2007.

[94] P. Di Fulvio et al., "Features of endothelial dysfunction in umbilical cord vessels of women with gestational diabetes," Nutr. Metab. Cardiovasc. Dis., vol. 24, no. 12, pp. 1337-1345, Dec. 2014.

[95] X. L. Du, D. Edelstein, S. Dimmeler, Q. Ju, C. Sui, and M. Brownlee, "Hyperglycemia inhibits endothelial nitric oxide synthase activity by posttranslational modification at the Akt site.,"J. Clin. Invest., vol. 108, no. 9, pp. 1341-8, Nov. 2001.

[96] F. Cosentino, K. Hishikawa, Z. S. Katusic, and T. F. Lüscher, "High glucose increases nitric oxide synthase expression and superoxide anion generation in human aortic endothelial cells.," Circulation, vol. 96, no. 1, pp. 25-8, Jul. 1997.

[97] H. Patel, J. Chen, K. C. Das, and M. Kavdia, "Hyperglycemia induces differential change in oxidative stress at gene expression and functional levels in HUVEC and HMVEC," Cardiovasc. Diabetol., vol. 12, no. 1, p. 142, Oct. 2013.

[98] S. C. Rogers, X. Zhang, G. Azhar, S. Luo, and J. Y. Wei, "Exposure to high or low glucose levels accelerates the appearance of markers of endothelial cell senescence and induces dysregulation of nitric oxide synthase.," J. Gerontol. A. Biol. Sci. Med. Sci., vol. 68, no. 12, pp. 1469-81, Dec. 2013.

[99] L. Piconi et al., "Constant and intermittent high glucose enhances endothelial cell apoptosis through mitochondrial superoxide overproduction," Diabetes. Metab. Res. Rev., 
vol. 22, no. 3, pp. 198-203, May 2006.

[100] Y. Chen, J. Chen, J. Hu, Z. Yang, and Y. Shen, "Enhancement of lipopolysaccharideinduced toll-like receptor 2 expression and inflammatory cytokine secretion in HUVECs under high glucose conditions," Life Sci., vol. 92, no. 10, pp. 582-588, Mar. 2013.

[101] H. Matsui-Hirai et al., "Dose-dependent modulatory effects of insulin on glucose-induced endothelial senescence in vitro and in vivo: a relationship between telomeres and nitric oxide.," J. Pharmacol. Exp. Ther., vol. 337, no. 3, pp. 591-9, Jun. 2011.

[102] T. Yu, J. L. Robotham, and Y. Yoon, "Increased production of reactive oxygen species in hyperglycemic conditions requires dynamic change of mitochondrial morphology.," Proc. Natl. Acad. Sci. U. S. A., vol. 103, no. 8, pp. 2653-8, Feb. 2006.

[103] D. Yao and M. Brownlee, "Hyperglycemia-induced reactive oxygen species increase expression of the receptor for advanced glycation end products (RAGE) and RAGE ligands.," Diabetes, vol. 59, no. 1, pp. 249-55, Jan. 2010.

[104] K.-L. Eskla et al., "Hypothermia augments stress response in mammalian cells," Free Radic. Biol. Med., vol. 121, pp. 157-168, Jun. 2018.

[105] G. Dijkstra et al., "Expression of nitric oxide synthases and formation of nitrotyrosine and reactive oxygen species in inflammatory bowel disease," J. Pathol., vol. 186, no. 4, pp. 416-421, Dec. 1998.

[106] H. F. G. Heijnen et al., "Subcellular localization of tyrosine-nitrated proteins is dictated by reactive oxygen species generating enzymes and by proximity to nitric oxide synthase," Free Radic. Biol. Med., vol. 40, no. 11, pp. 1903-1913, Jun. 2006.

[107] L. Monnier et al., "Activation of Oxidative Stress by Acute Glucose Fluctuations Compared With Sustained Chronic Hyperglycemia in Patients With Type 2 Diabetes," 
JAMA, vol. 295, no. 14, p. 1681, Apr. 2006.

[108] K. Esposito et al., "Inflammatory cytokine concentrations are acutely increased by hyperglycemia in humans: role of oxidative stress.," Circulation, vol. 106, no. 16, pp. 2067-72, Oct. 2002.

[109] A. Ceriello, "The emerging role of post-prandial hyperglycaemic spikes in the pathogenesis of diabetic complications," Diabet. Med., vol. 15, no. 3, pp. 188-193, Mar. 1998.

[110] S. Kerkar, M. Williams, J. M. Blocksom, R. F. Wilson, J. G. Tyburski, and C. P. Steffes, “TNF- $\alpha$ and IL-1 $\beta$ Increase Pericyte/Endothelial Cell Co-Culture Permeability,” J. Surg. Res., vol. 132, no. 1, pp. 40-45, May 2006.

[111] V. Modur, G. A. Zimmerman, S. M. Prescott, and T. M. McIntyre, "Endothelial cell inflammatory responses to tumor necrosis factor alpha. Ceramide-dependent and independent mitogen-activated protein kinase cascades.," J. Biol. Chem., vol. 271, no. 22, pp. 13094-102, May 1996.

[112] Y. Cao, J. Zhang, X. Meng, and D. Wang, "TNF- $\alpha$ induces early growth response gene-1 expression via ERK1/2 activation in endothelial cells," Acta Diabetol., vol. 50, no. 1, pp. 27-31, Feb. 2013.

[113] J. Zhang, G. Wu, and H. Dai, "The matricellular protein CCN1 regulates TNF- $\alpha$ induced vascular endothelial cell apoptosis," Cell Biol. Int., vol. 40, no. 1, pp. 1-6, Jan. 2016.

[114] S. Varma et al., "Hyperglycemia alters PI3k and Akt signaling and leads to endothelial cell proliferative dysfunction," Am. J. Physiol. Circ. Physiol., vol. 289, no. 4, pp. H1744H1751, Oct. 2005.

[115] Y. Zhang et al., "Interleukin-6 is required for pancreatic cancer progression by promoting 
MAPK signaling activation and oxidative stress resistance.," Cancer Res., vol. 73, no. 20, pp. 6359-74, Oct. 2013. 


\section{APPENDIX A: PRIMER SEQUENCES}

Primer Sequences

Custom Primers, IDT DNA "Custom Oligos"

\begin{tabular}{|c|c|c|}
\hline Gene Name & Primer Sequences & Amplification? \\
\hline IL-6 & $\begin{array}{l}\text { FOR 5'CCTTCTCCACAAACATGTAACAAGA3' } \\
\text { REV 5'TCACCAGGCAAGTCTCCTCA3' }\end{array}$ & $\mathrm{N}$ \\
\hline IL-6 & $\begin{array}{l}\text { FOR 5'TGTTACATGTTTGTGGAGAAGGAG3' } \\
\text { REV 5'CTCAATATTAGAGTCTCAACCCCCA3' }\end{array}$ & $\mathrm{N}$ \\
\hline CD36 & $\begin{array}{l}\text { FOR 5'AAGACTTTCCTGCAGAATACCA3' } \\
\text { REV 5' ACAAGCTCTGGTTCTTATTCACA3' }\end{array}$ & $\mathrm{N}$ \\
\hline CD36 & $\begin{array}{l}\text { FOR 5'TTCTGCATCTGCTCCTGCAA3' } \\
\text { REV 5'ACAAGCTATGGTTCTTATTCACA3' }\end{array}$ & $\mathrm{N}$ \\
\hline CD68 & $\begin{array}{l}\text { FOR 5'TAGCTGGACTTTGGGTGAGG3' } \\
\text { REV 5'TAGCTGGACTTTGGGTGAGG3' }\end{array}$ & $\mathrm{Y}$ \\
\hline CD68 & $\begin{array}{l}\text { FOR 5'CCAGTGCTCTCTGCCAGTA3' } \\
\text { REV 5'GGTGGGATCATCTCCAGTACA3' }\end{array}$ & $\mathrm{N}$ \\
\hline IL-1A & $\begin{array}{l}\text { FOR 5'GGCCATCTTGACTTCTTTGCT3' } \\
\text { REV 5'GACGCACTTGTAGCCACGTA3' }\end{array}$ & $\mathrm{N}$ \\
\hline IL-1A & $\begin{array}{l}\text { FOR 5'AGGCTTGATGATTTCTTCCTCTGA3' } \\
\text { REV 5'AGTAGCAACCAACGGGAAGG3' }\end{array}$ & $\mathrm{Y}$ \\
\hline PLA2G7 & $\begin{array}{l}\text { FOR 5'TAAGTTAACCGCGGGTCCAG3' } \\
\text { REV 5'TTGTTGACCCATGCTGATGA3' }\end{array}$ & $\mathrm{N}$ \\
\hline PLA2G7 & FOR 5'GCATCAGGTCTGCGGAAAGG3' & $\mathrm{N}$ \\
\hline
\end{tabular}




\begin{tabular}{|l|l|l|}
\hline & REV 5'TTTCAGCTTAGTCTCCTGGACC3' & \\
\hline PTGS2 & $\begin{array}{l}\text { FOR 5'CAAATTGCTGGCAGGGTTGC3' } \\
\text { REV 5'AGGGCTTCAGCATAAAGCGT3' }\end{array}$ & Y \\
\hline PTGS2 & $\begin{array}{l}\text { FOR 5'TCCCTTGGGTGTCAAAGGTAAA3' } \\
\text { REV 5'TGCCCCTCGCTTATGATCTG3' }\end{array}$ & Y \\
\hline PPARG & FOR 5'CGTGGCCGCAGAAATGAC3' & Low \\
\hline PPARG & FEV 5'CACGGAGCTGATCCCAAAGT3' 5'GCTTGGGTCGGCCTCG3' & \\
\hline & REV 5'CAACCATGGTCATTTCTGCGG3' & N \\
\hline
\end{tabular}


PrimeTime ${ }^{\circledR}$ Std qPCR Assays ${ }^{\ddagger}$, IDT DNA

\begin{tabular}{|c|c|}
\hline Gene & Sequences \\
\hline ACTB & $\begin{array}{l}\text { Probe 5'/56-FAM/TCATCCATG/ZEN/GTGAGCTGGCGG/3IABkFQ/-3' } \\
\text { Primer } 1 \text { 5'-CCTTGCACATGCCGGAG-3' } \\
\text { Primer 2 5'-ACAGAGCCTCGCCTTTG-3' }\end{array}$ \\
\hline B2M & $\begin{array}{l}\text { Probe 5'-/56-FAM/CTGCCGTG/ZEN/TGAACCATGTGACT/3IABkFQ/-3' } \\
\text { Primer } 1 \text { 5'-ACCTCCATGATGCTGCTTAC-3' } \\
\text { Primer } 2 \text { 5'-GGACTGGTCTTTCTATCTCTTGT-3' }\end{array}$ \\
\hline CD36 & $\begin{array}{l}\text { Probe 5'-/56-FAM/TGTAGGACC/ZEN/TCAAGACTGGCTCCA/3IABkFQ/3' } \\
\text { Primer } 1 \text { 5'-TCAATTCGTCTAATCATTGGAAAGC-3' } \\
\text { Primer } 2 \text { 5'-CCTGTTTACTTTCTGCATCTGC-3' }\end{array}$ \\
\hline HPRT1 & $\begin{array}{l}\text { Probe 5'-/56- } \\
\text { FAM/AGCCTAAGA/ZEN/TGAGAGTTCAAGTTGAGTTTGG/3IABkFQ/-3' } \\
\text { Primer } 1 \text { 5'-GCGATGTCAATAGGACTCCAG-3' } \\
\text { Primer } 2 \text { 5'-TTGTTGTAGGATATGCCCTTGA-3' }\end{array}$ \\
\hline IL-6 & $\begin{array}{l}\text { Probe 5'-/56-FAM/CAACCACAA/ZEN/ATGCCAGCCTGCT/3IABkFQ/-3' } \\
\text { Primer } 1 \text { 5'-TTCTGTGCCTGCAGCTTC-3' } \\
\text { Primer } 2 \text { 5'-GCAGATGAGTACAAAAGTCCTGA-3' }\end{array}$ \\
\hline
\end{tabular}

\$ Be sure to select "Primers Only" when ordering to avoid buying probes. Use SYBR reporter instead. 


\section{APPENDIX B: PROTOCOL FOR PRIMER DESIGN}

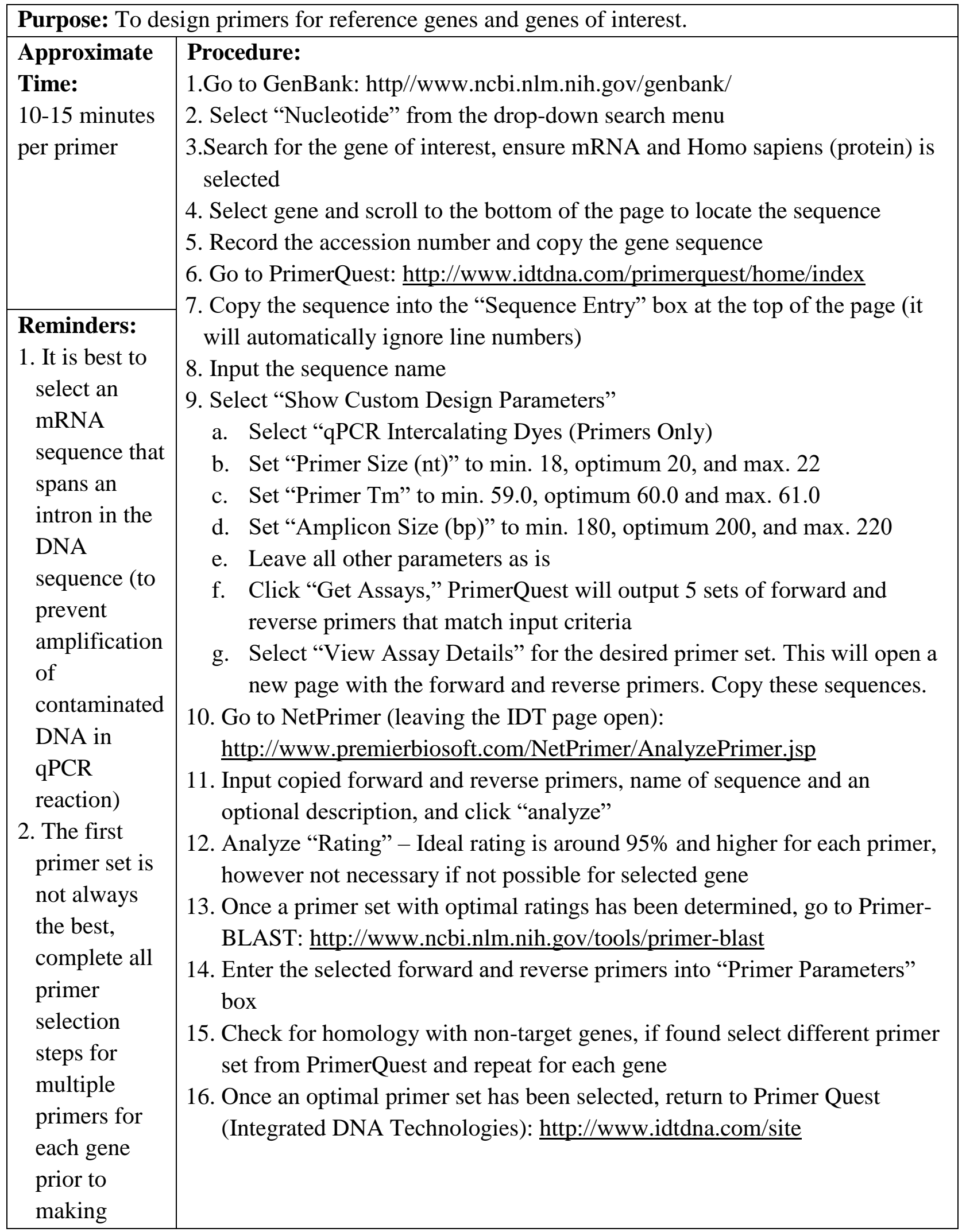




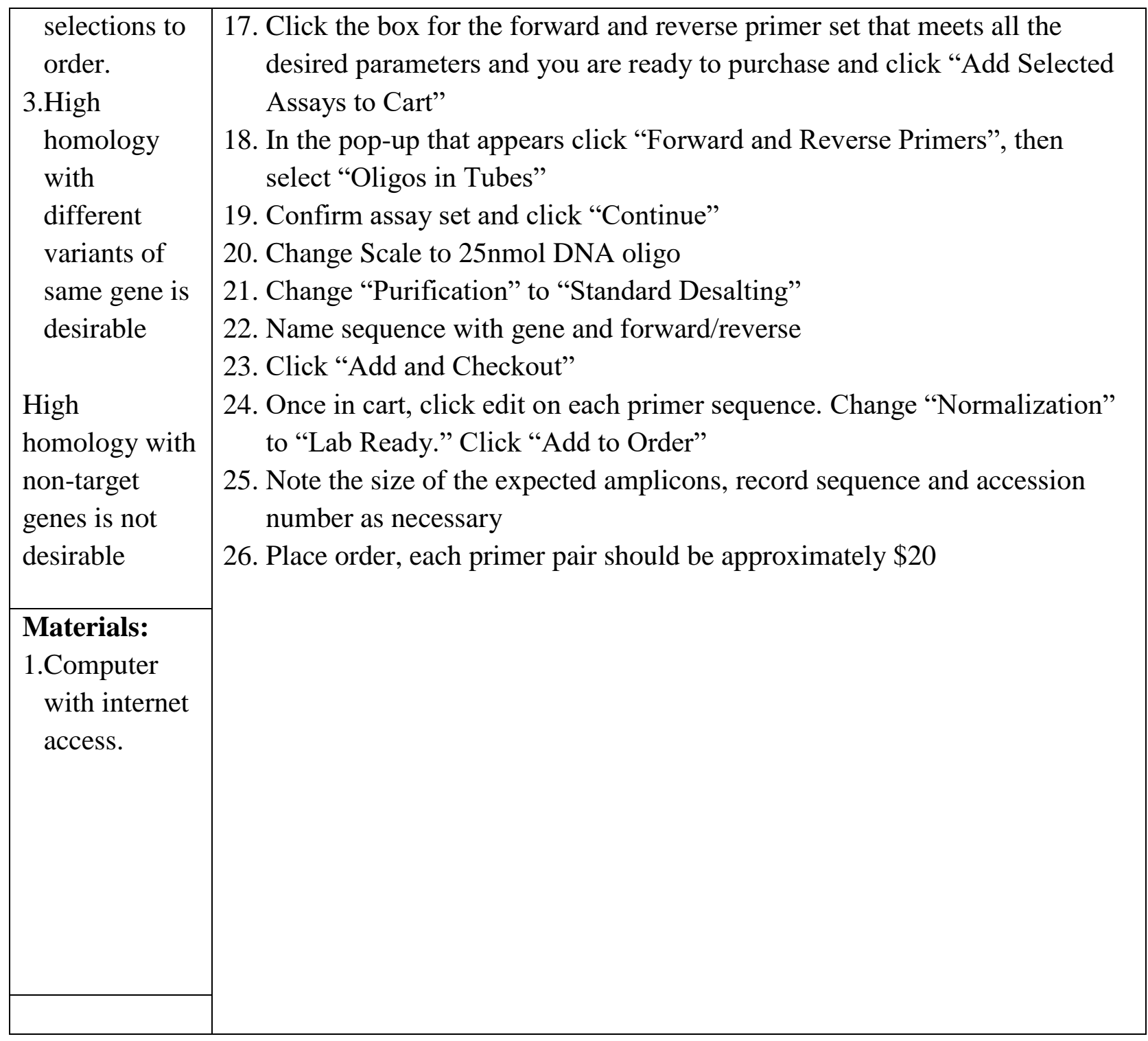

\begin{tabular}{|l|l|l|}
\hline \multicolumn{2}{|l|}{ Revision History } \\
\hline A & $\begin{array}{l}\text { Michael Gibbons } \\
\text { Sarah Ur }\end{array}$ & \\
\hline B & Tristin McGuffick & 140605 \\
\hline C & Shelby Kunz & 160301 \\
\hline D & Gabriella Sugerman & 180707 \\
\hline
\end{tabular}




\section{APPENDIX C: PROTOCOL FOR QPCR}

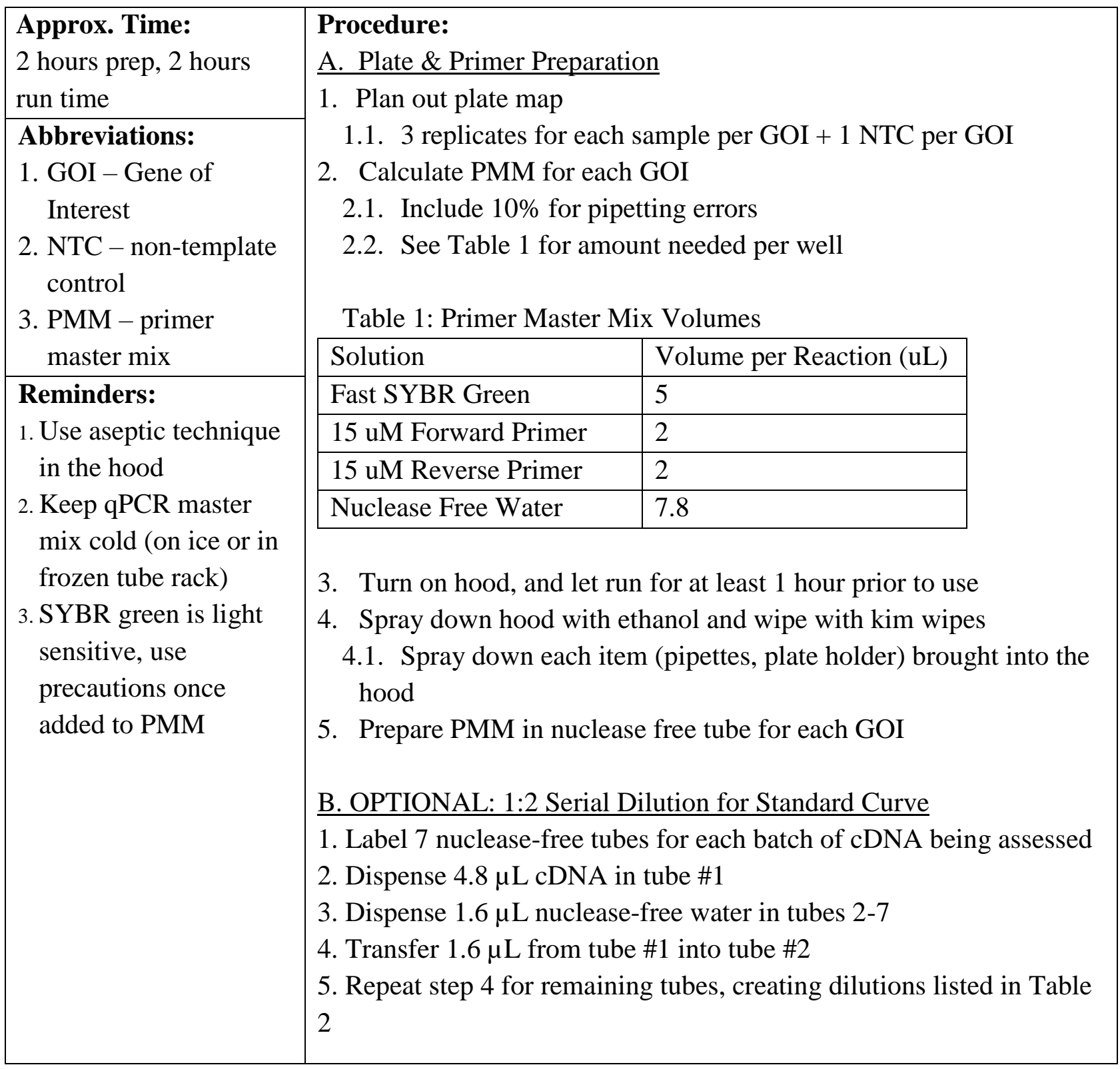




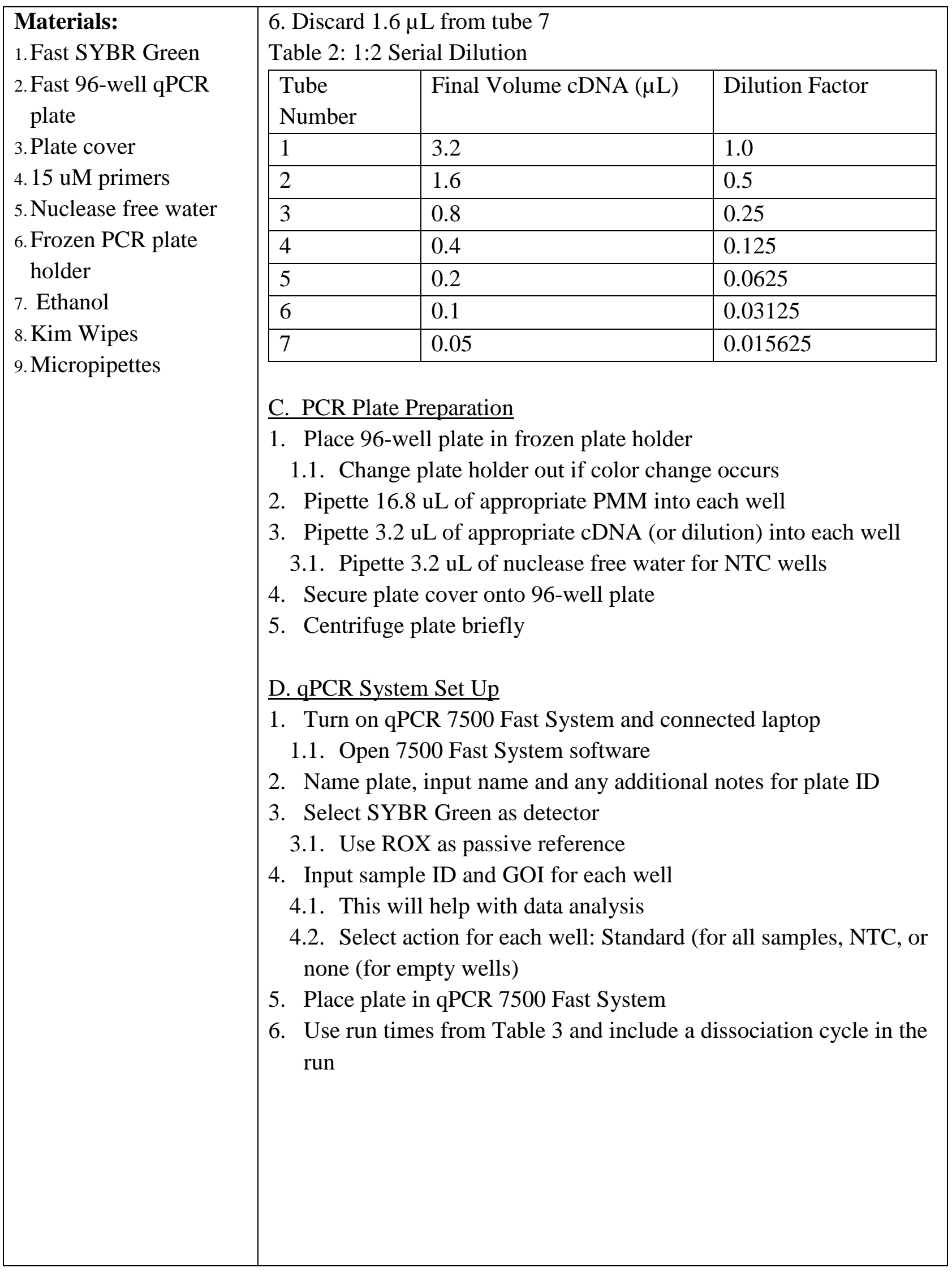




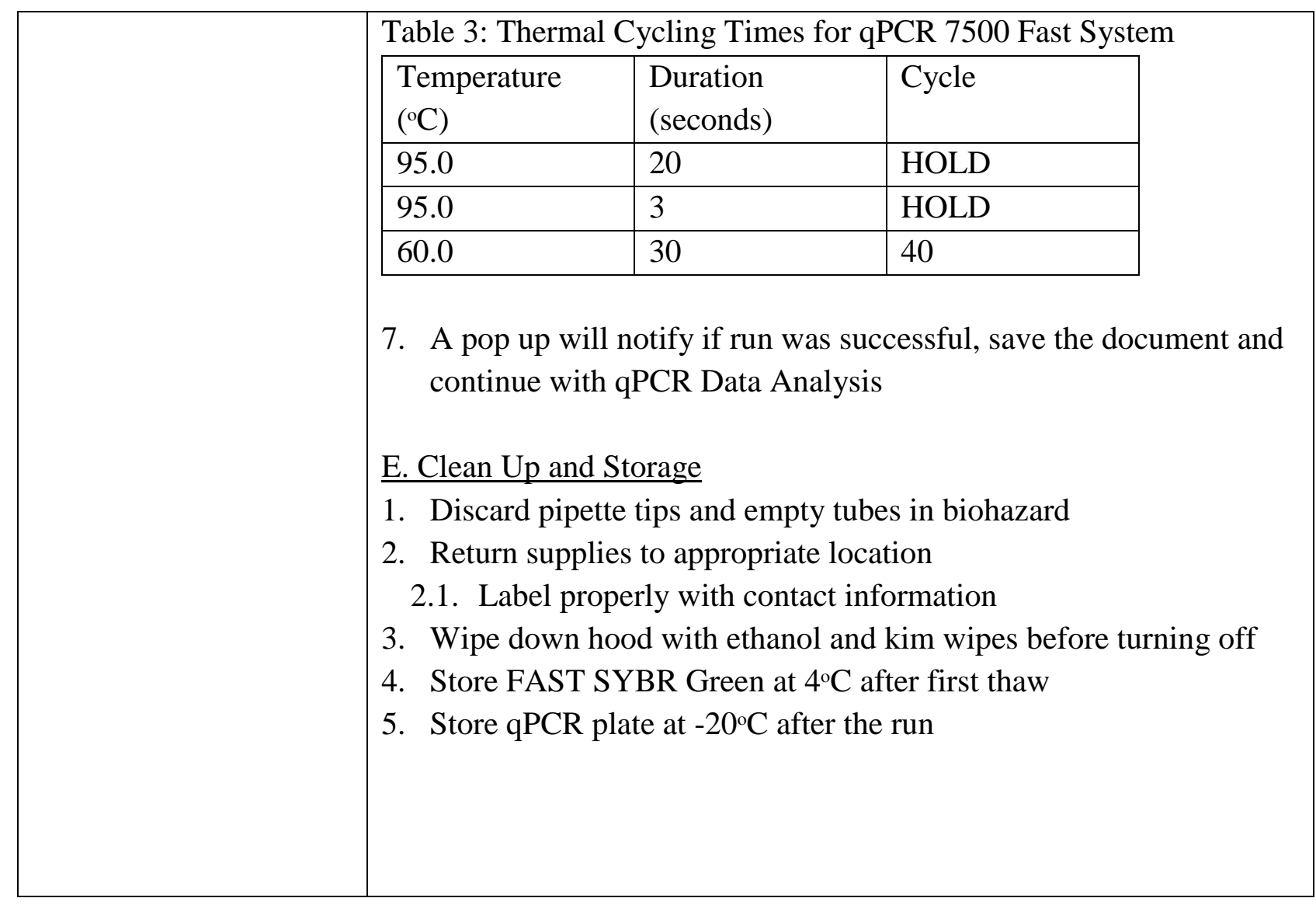

\begin{tabular}{|l|l|l|}
\hline \multicolumn{3}{|l|}{ Revision History } \\
\hline A & $\begin{array}{l}\text { Michael Gibbons } \\
\text { Sarah Ur }\end{array}$ & 120608 \\
\hline B & Tristin McGuffick & 140605 \\
\hline C & Shelby Kunz & 160601 \\
\hline D & Conor Hedigan & 171101 \\
\hline E & Gabriella Sugerman & 180707 \\
\hline
\end{tabular}




\section{APPENDIX D: PROTOCOL FOR QPCR DATA ANALYSIS}

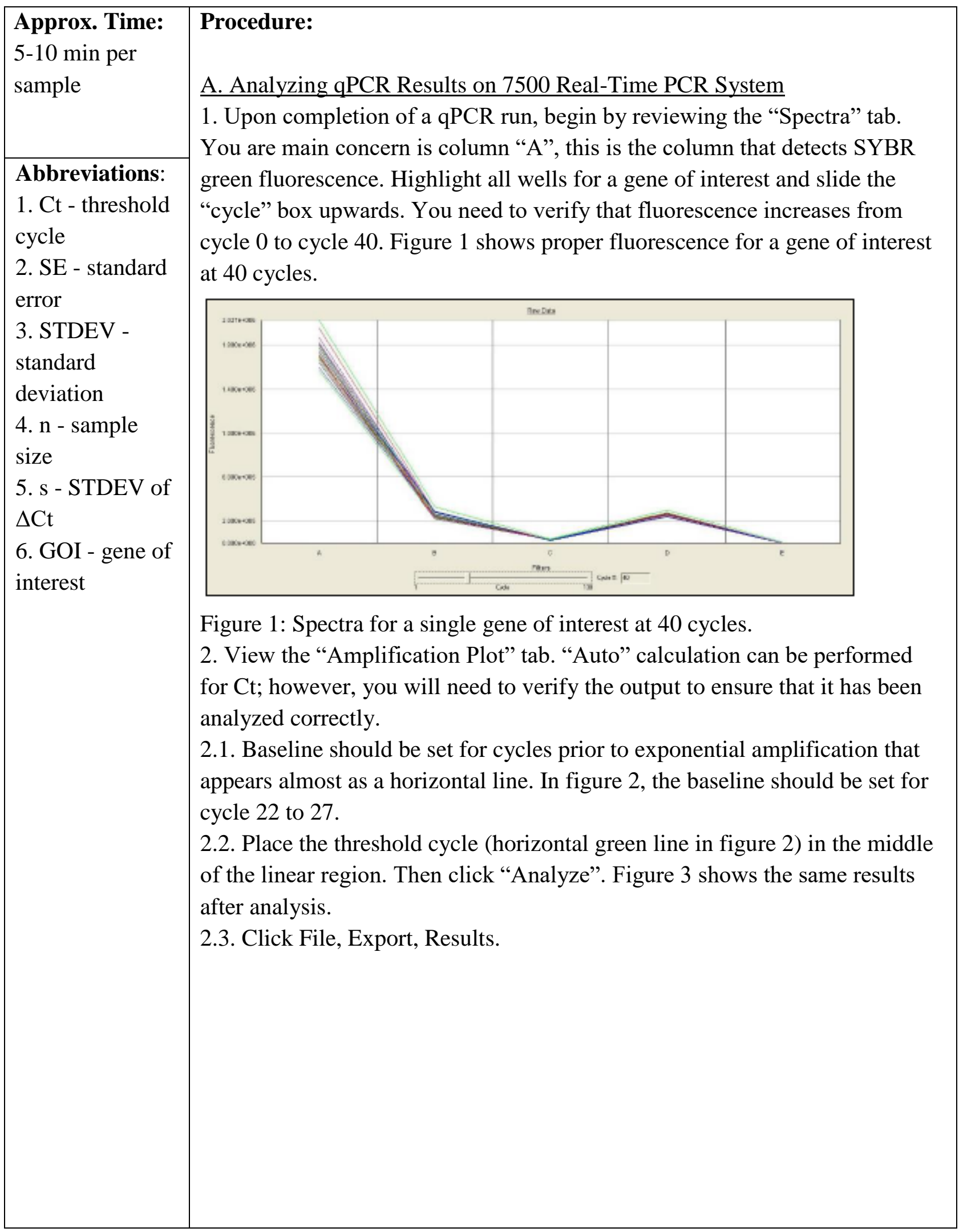




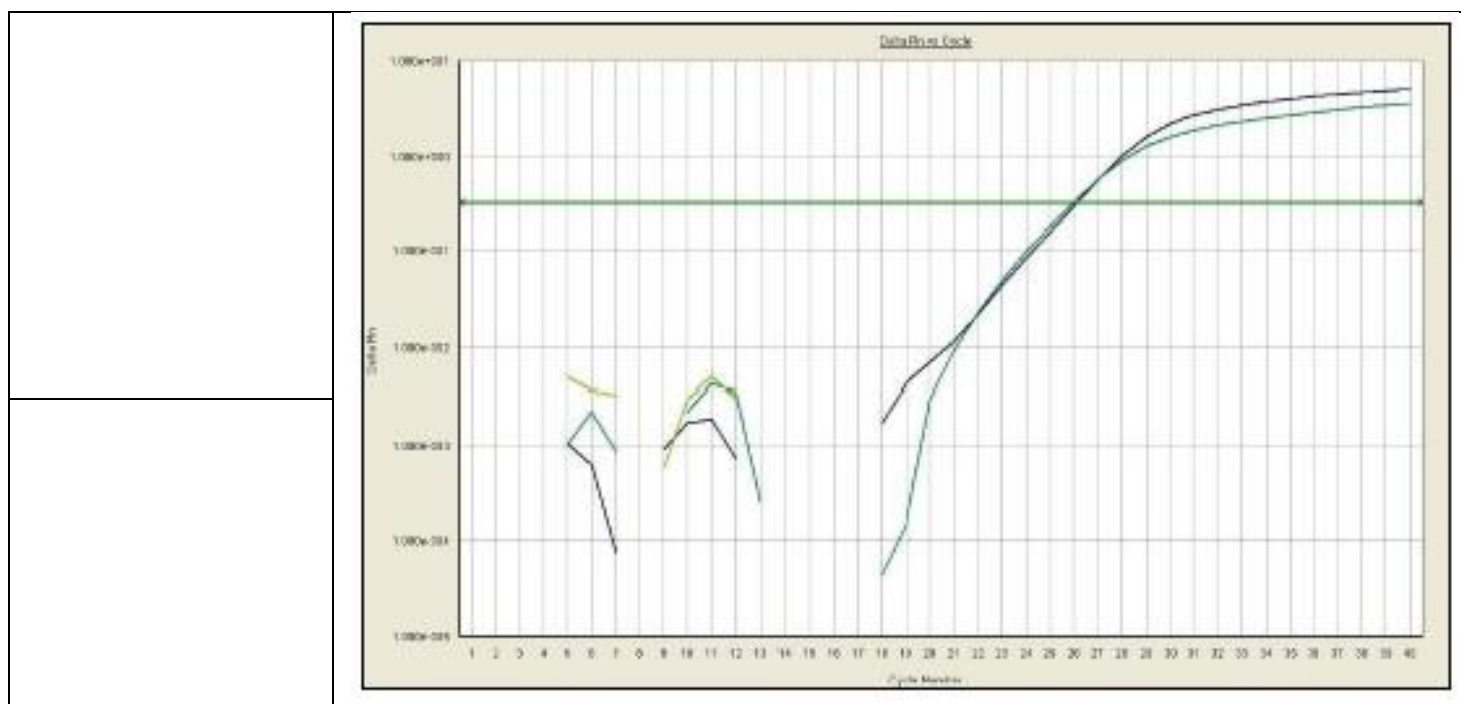

Figure 2: Raw amplification plot for a specific GOI.

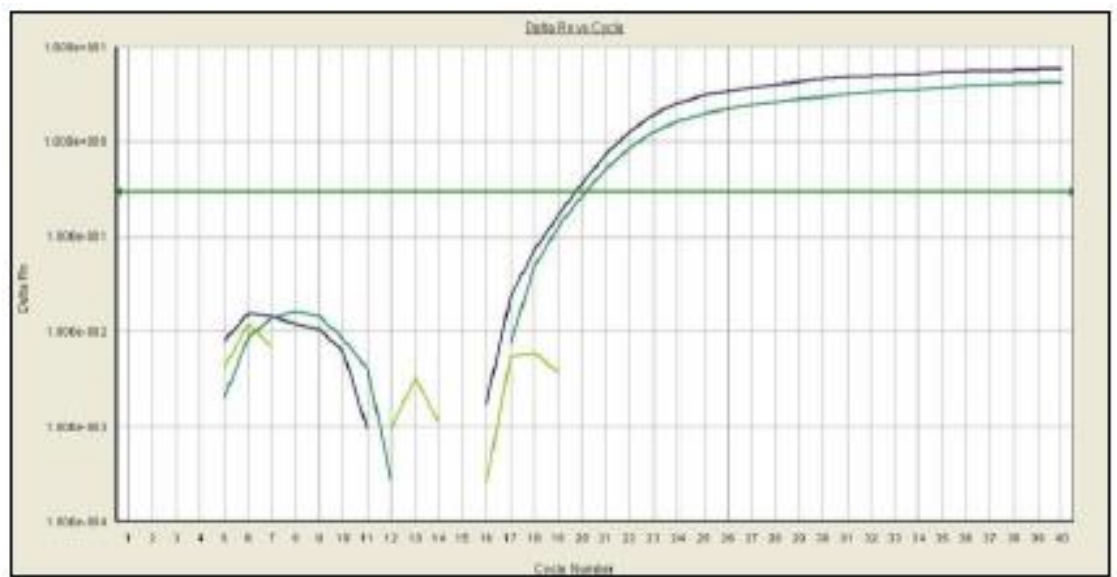

Figure 3: Amplification plot after being analyzed.

3. Next, click the "Dissociation Curve" tab. Ensure that all wells containing samples are melting at the same temperature, as seen in Figure 4.

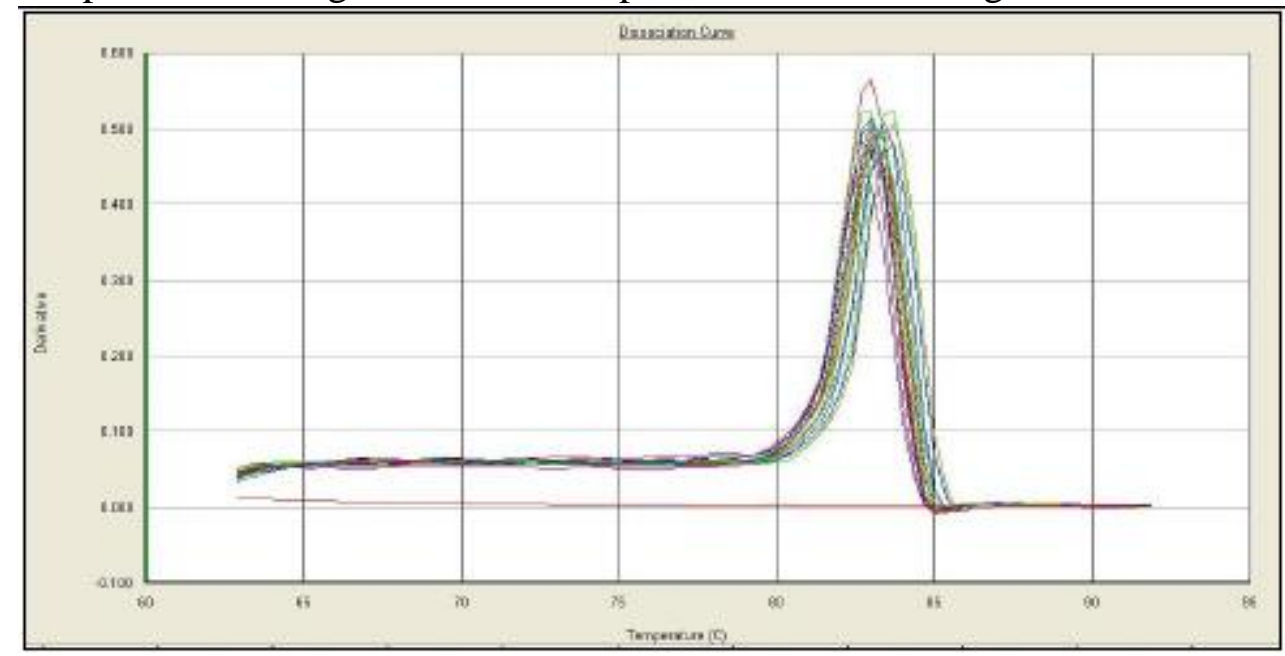

Figure 4: Ideal dissociation curve for a specific primer. 


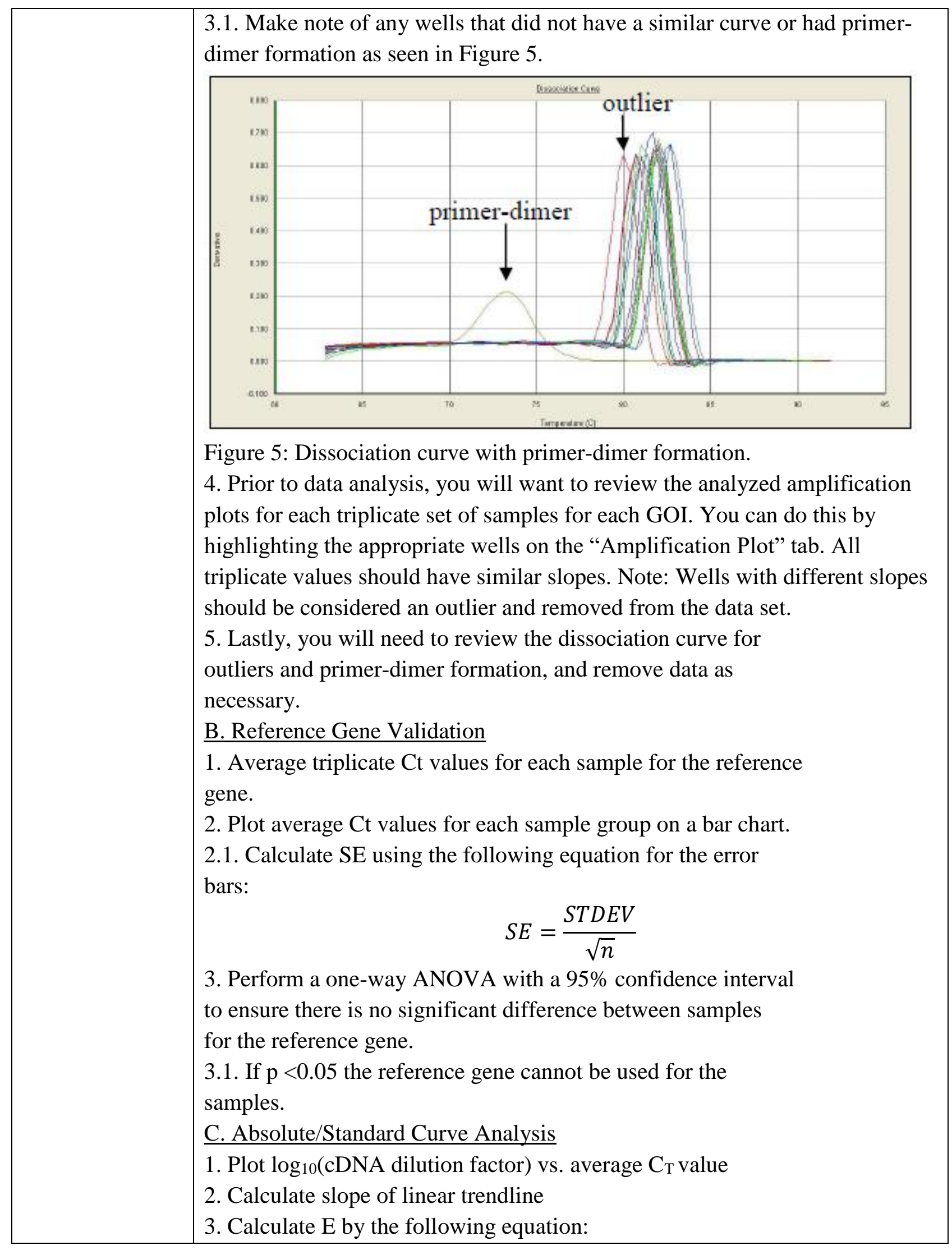




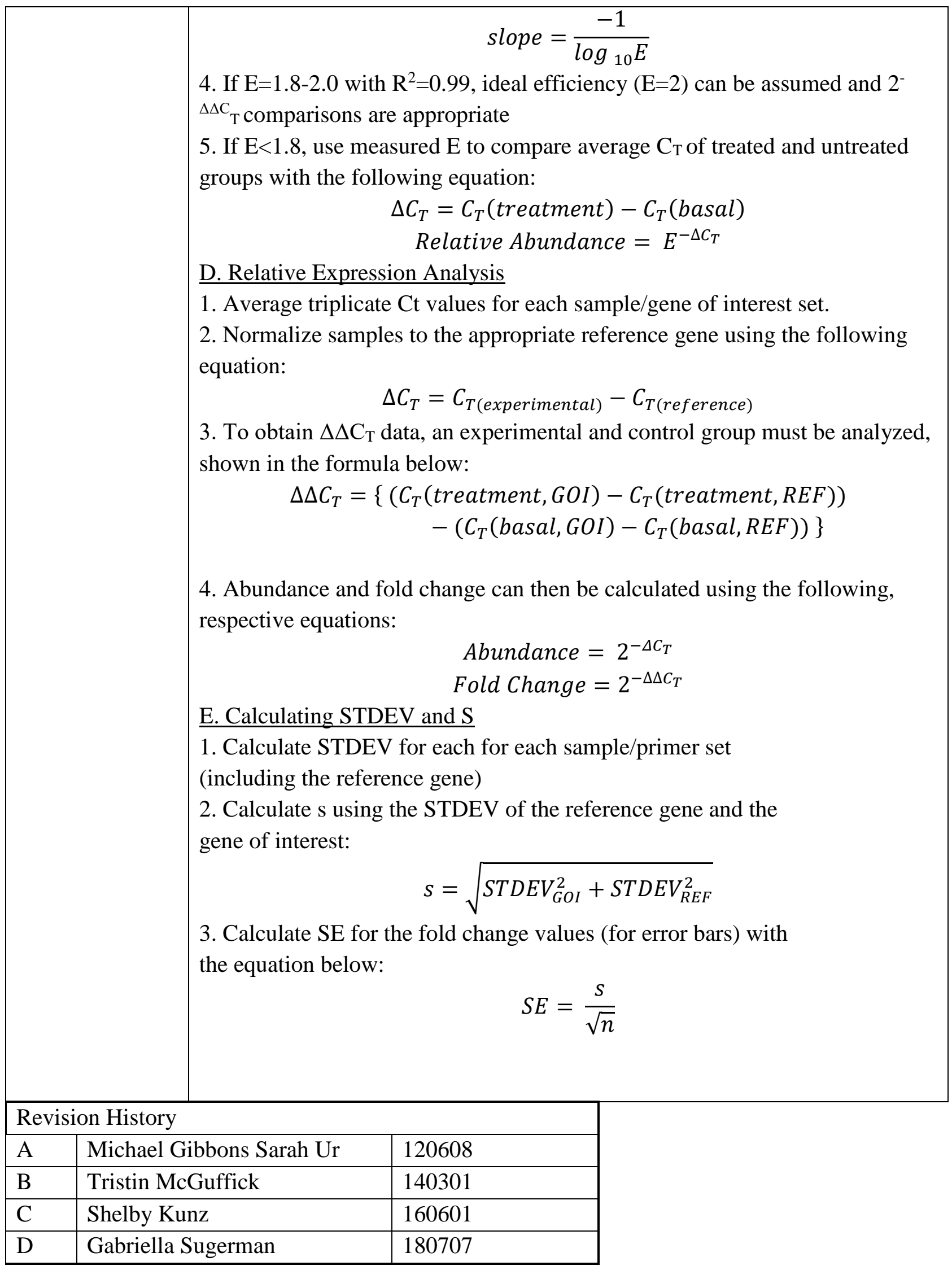




\section{APPENDIX E: PROTOCOLS FOR CELL CULTURE}

Purpose: The purpose of this SOP is to guide the user in thawing and feeding cells during culturing.

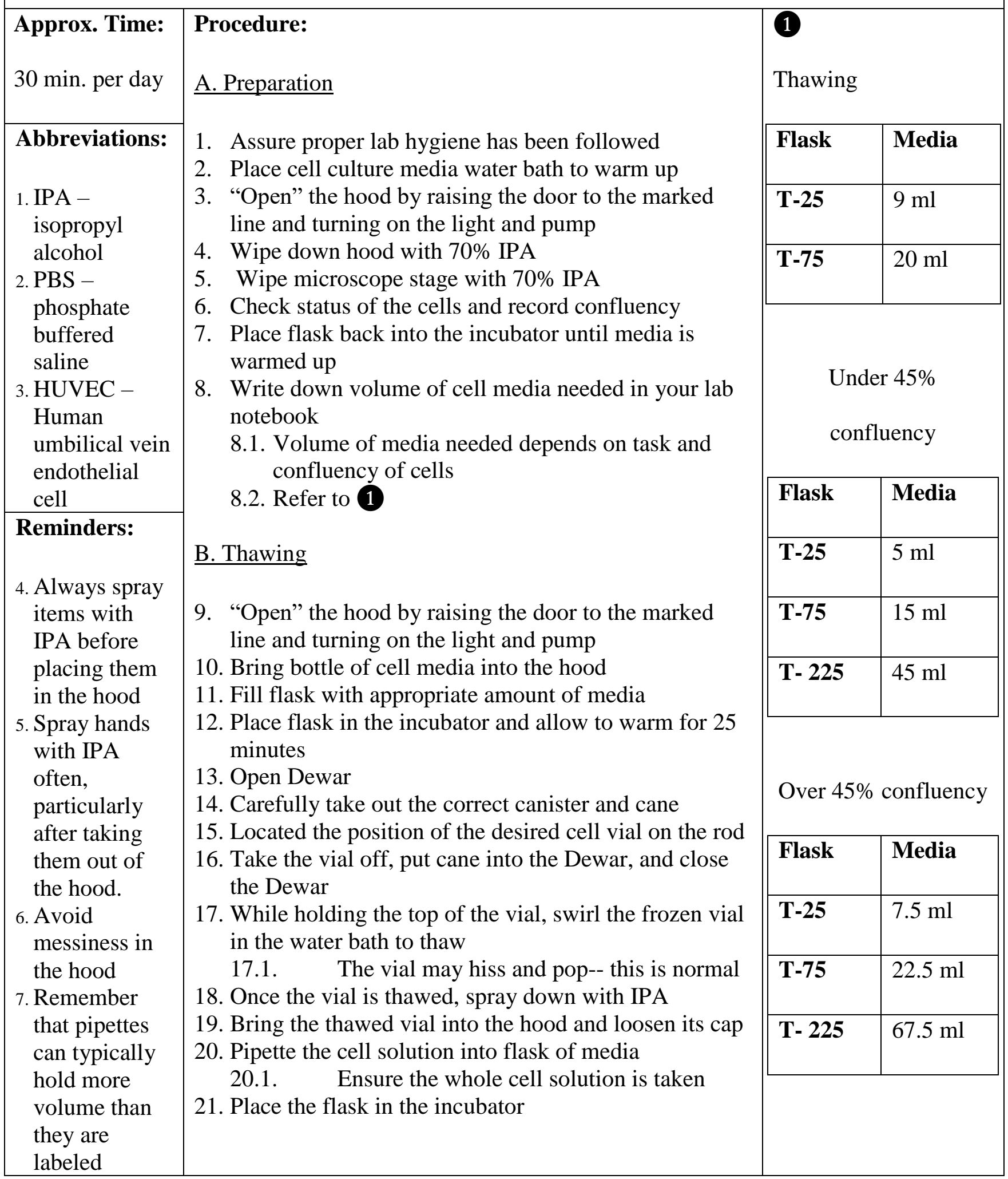




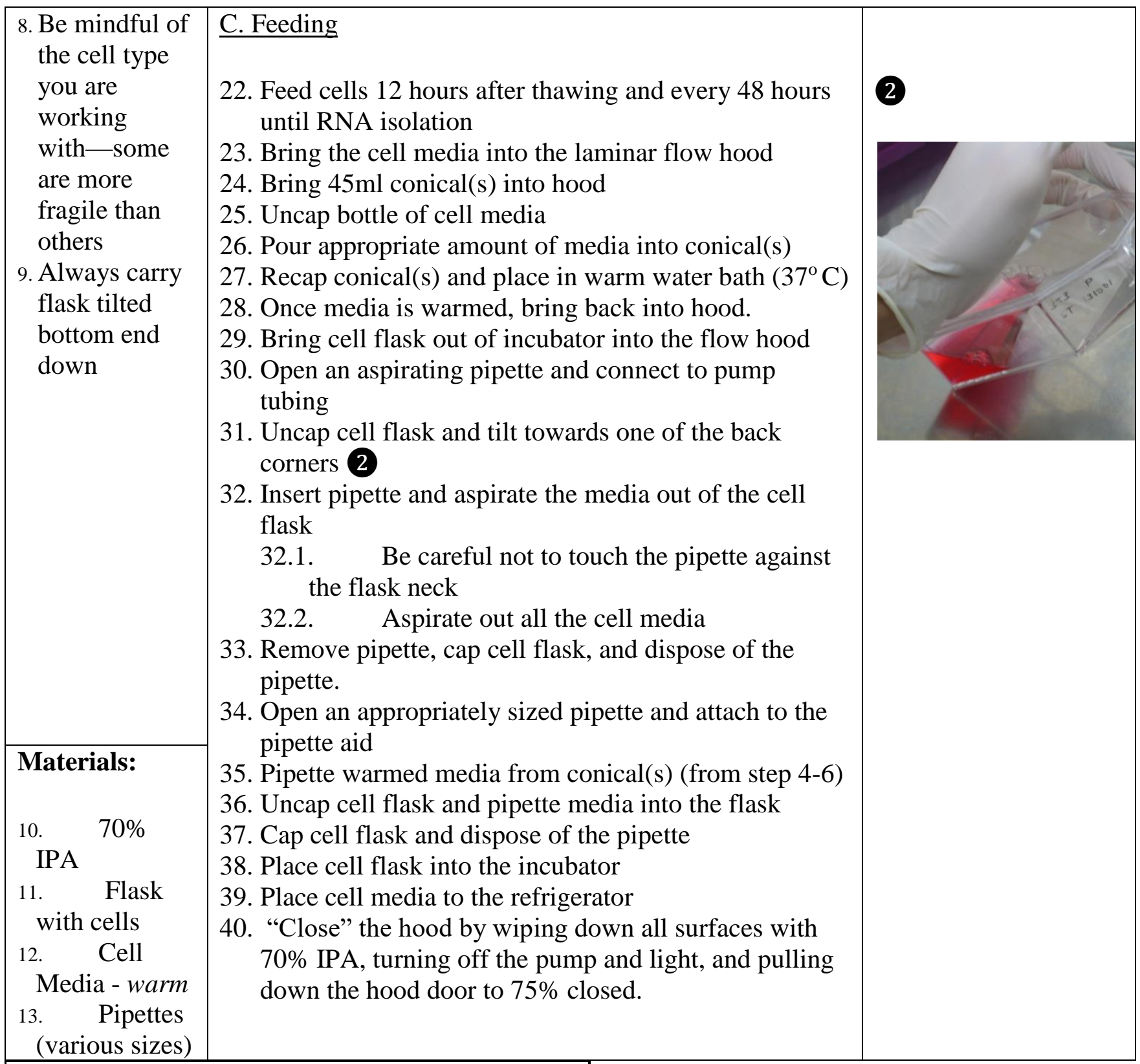

\section{Revision History:}

\begin{tabular}{|l|l|l|}
\hline A & Jakub Truty & Spring 2014 \\
\hline B & Shane Wilsey & Fall 2017 \\
\hline C & Gabriella Sugerman & 180708 \\
\hline
\end{tabular}




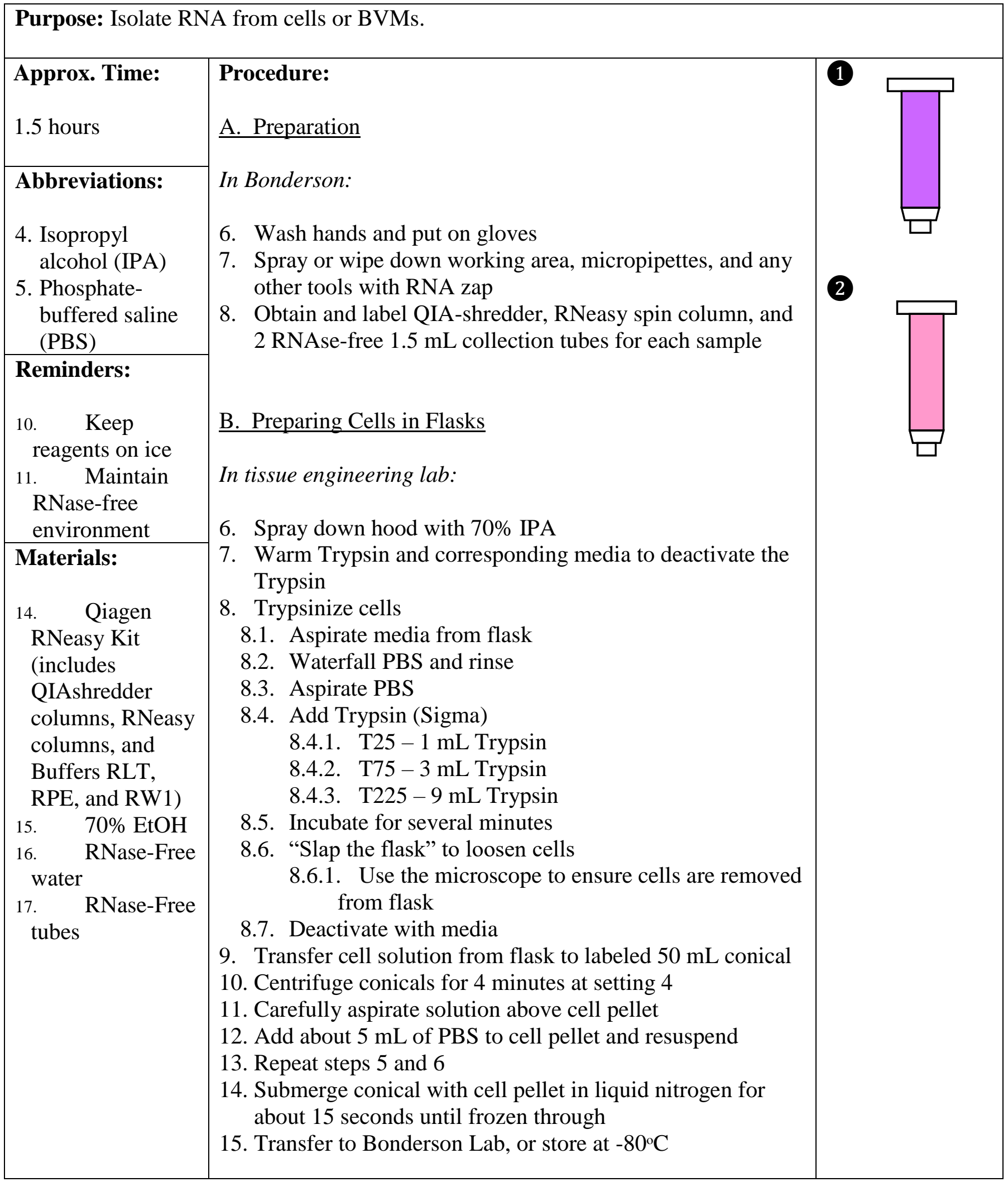




\section{Preparing Cells in BVM}

In Bonderson:

1. Record BVM dimensions

2. Rinse BVM in PBS to remove all traces of media

3. Transfer BVM to $1.5 \mathrm{~mL}$ collection tubes

D. RNA Isolation

\section{In Bonderson:}

1. Add $600 \mathrm{uL}$ of Buffer RLT to sample

1.1. Pipette up and down to mix

1.2. Vortex tube for 2 minutes

1.3. For BVMs, add half the volume of Buffer RLT and perform steps 1.1 and 1.2 twice

2. Transfer all cell lysate to QIA shredder spin column (1)

2.1. Centrifuge at $16.1 \times 10^{4} \mathrm{rcf}$ for 2 minutes

3. Add 600 ul of $70 \%$ ethanol to flow through from QIA shredder spin column

3.1. Pipette up and down to mix

4. Transfer $600 \mathrm{uL}$ of flow through solutions to RNeasy spin column 2

4.1. Centrifuge at $16.1 \times 10^{4} \mathrm{rcf}$ for 30 seconds

4.2. Discard flow through in waste

4.3. Repeat step 4 for the remaining flow through from QIA shredder spin column

5. Add 700 uL RW 1 washing buffer to RNeasy spin column

5.1. Centrifuge at $16.1 \times 10^{4} \mathrm{rcf}$ for 30 seconds

5.2. Discard flow through in waste

6. Add $500 \mathrm{uL}$ Buffer RPE to RNeasy spin column

6.1. Centrifuge at $16.1 \times 10^{4}$ ref for 30 seconds

6.2. Discard flow through in waste

7. Add $500 \mathrm{uL}$ Buffer RPE to RNeasy spin column

7.1. Let sit for 5 minutes

7.2. Centrifuge at $16.1 \times 10^{4} \mathrm{rcf}$ for 2 minutes

7.3. Discard flow through in waste

8. Centrifuge empty RNeasy spin column at $16.1 \times 10^{4} \mathrm{rcf}$ for 2 minutes

9. Change gloves and wipe down with RNA zap wipes 


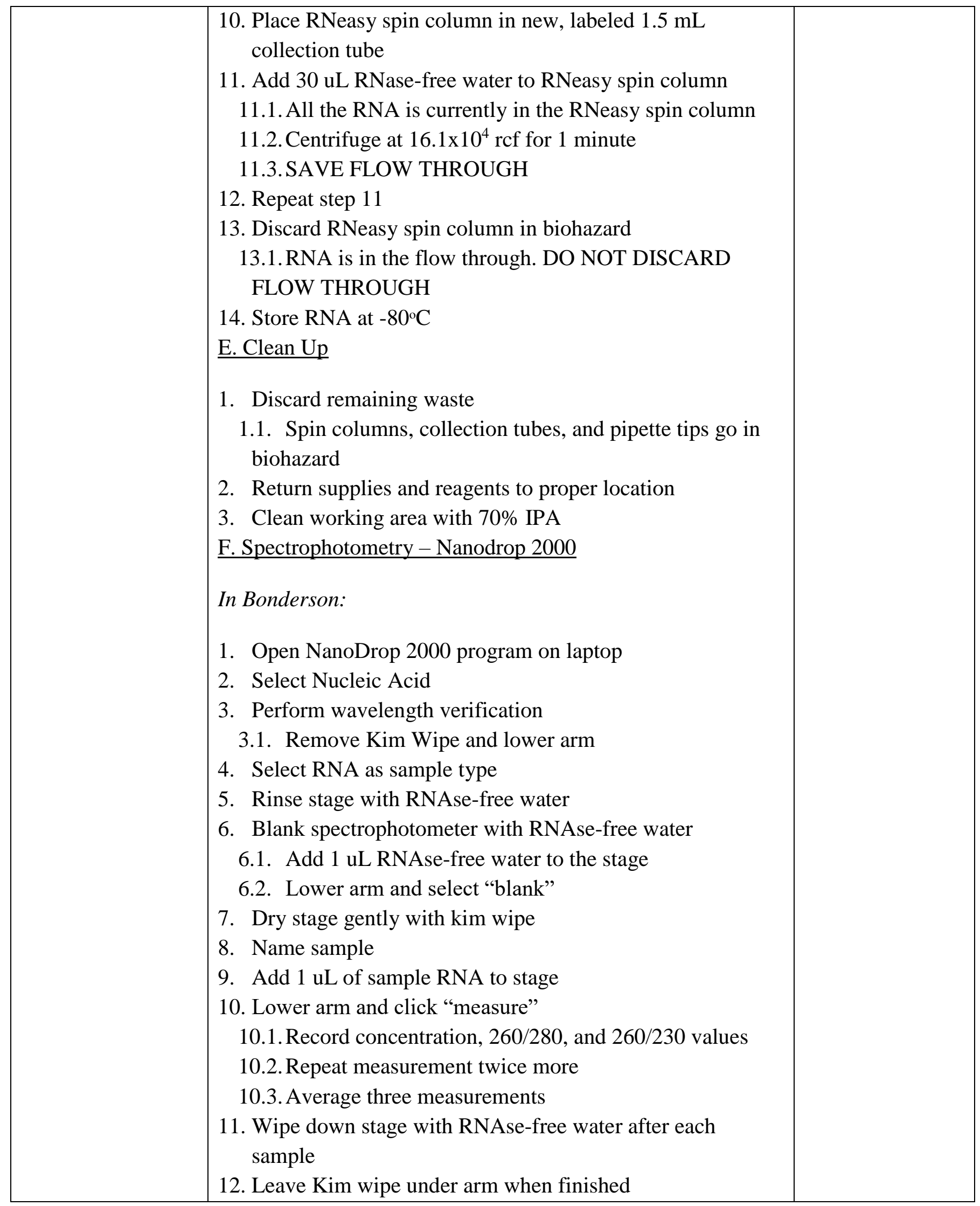




\begin{tabular}{|l|l|}
\hline 13. If the concentration of the RNA is high, it may be \\
necessary to dilute the sample \\
13.1. It is difficult to accurately pipette less than $1 \mathrm{uL}$ \\
values, so dilution will allow for greater pipetting accuracy \\
13.2. Add small volumes of RNAse-free water to the RNA \\
sample \\
13.3. Test the concentration of the sample after each dilution \\
until appropriate concentration is reached \\
\end{tabular} \mid

\begin{tabular}{|l|l|l|}
\hline \multicolumn{2}{|l|}{ Revision History } & 120608 \\
\hline A & Michael Gibbons & \\
\hline B & Sarah Ur & 150122 \\
\hline C & Shelby Kunz & 160601 \\
\hline D & Conor Hedigan & 171101 \\
\hline E & Gabriella Sugerman & 180531 \\
\hline
\end{tabular}




\section{APPENDIX G: PROTOCOL FOR CDNA SYNTHESIS}

\begin{tabular}{|c|c|}
\hline $\begin{array}{l}\text { Approx. Time: } \\
1.5 \text { hours }\end{array}$ & $\begin{array}{l}\text { Procedure: } \\
\text { A. Preparation }\end{array}$ \\
\hline $\begin{array}{l}\text { Abbreviations: } \\
\text { 1. } \mathrm{RT} \text { - reverse } \\
\text { transcriptase }\end{array}$ & $\begin{array}{l}\text { 1. Using average concentration obtained in RNA assessment, } \\
\text { calculate volume of RNA needed } \\
\text { 1.1. Determine amount of RNA (ng) for duration of experiment }\end{array}$ \\
\hline $\begin{array}{l}\text { Reminders: } \\
\text { 1. Keep working area } \\
\text { and tools RNAse } \\
\text { free } \\
\text { 2.Store iScript cDNA } \\
\text { synthesis kit at }-20^{\circ} \mathrm{C}\end{array}$ & $\begin{array}{l}\text { 1.2. } 200 \mathrm{ng} \text { to } 1000 \mathrm{ng} \text { are needed to run } \mathrm{qPCR} \\
\text { 1.2.1. We use } 1000 \mathrm{ng} \\
\text { 1.3. (x ng of RNA })^{*}(1 \mathrm{~mL} /[\mathrm{RNA} \mathrm{ng} / \mathrm{nL}])=\text { Volume RNA }(\mathrm{uL}) \\
\text { 2. Calculate amount of nuclease free water needed } \\
\text { 2.1. } 15 \mathrm{uL} \text { - Volume RNA = Volume } \mathrm{H}_{2} \mathrm{O}(\mathrm{uL}) \\
\text { 3. Wipe down working area, micropipettes, and any other tools } \\
\text { with RNAse Zap Wipes } \\
\text { B. iScript Transcription } \\
\text { 4. Prepare master mix } \\
\text { 4.1. } 4 \mathrm{uL} \text { of } 5 \mathrm{X} \text { iScript Reaction Mix per sample } \\
\text { 4.2. } 1 \mathrm{uL} \text { of RT per reaction } \\
\text { 4.3. Include additional } 10 \% \text { of each reagent for pipetting error } \\
\text { 4.4. Ex: for } 3 \text { samples, need } 13.2 \mathrm{uL} \text { of } 5 \mathrm{X} \text { iScript }+1.1 \mathrm{uL} \mathrm{RT}\end{array}$ \\
\hline
\end{tabular}




\begin{tabular}{|c|c|}
\hline $\begin{array}{l}\text { Materials: } \\
\text { 1. } \text { iScript cDNA } \\
\text { synthesis kit } \\
-\quad 5 x \text { iScript } \\
\quad \text { Reaction mix } \\
-\quad \text { RT } \\
-\quad \text { RNAse free } \\
\quad \text { water } \\
\text { 2. Nuclease free tubes } \\
\text { 3. Thermal cycler tubes }\end{array}$ & $\begin{array}{l}\text { 5. Label } 1.5 \mathrm{~mL} / 2 \mathrm{~mL} \text { nuclease free tubes for each sample } \\
\text { 6. Add } 5 \mathrm{uL} \text { of master mix to each tube } \\
\text { 7. Add calculated volume of RNA and nuclease free water to each } \\
\text { tube } \\
\text { 7.1. Centrifuge briefly } \\
\text { 8. Load samples }(20 \mathrm{uL} \text { ) into thermal cycler tubes and cap them. } \\
\text { 9. Place tubes into thermal cycler } \\
\text { 9.1. Ensure the lid of the thermal cycler is touching the caps of } \\
\text { the tubes } \\
\text { 10. Run thermal cycler program "ISCRIPT" } \\
\text { 10.1. } 25^{\circ} \mathrm{C} \text { for } 5 \text { minutes ( } 300 \text { seconds) } \\
\text { 10.2. } 42^{\circ} \mathrm{C} \text { for } 30 \text { minutes ( } 1800 \text { seconds) } \\
\text { 10.3. } 4^{\circ} \mathrm{C} \text { forever (hold) } \\
\text { 11. After cycling is completed, remove from thermal cycler } \\
\text { 12. Dilute cDNA } 1: 5 \text { with } 80 \mathrm{uL} \text { of nuclease free water } \\
\text { 13. Store cDNA at }-20^{\circ} \mathrm{C}\end{array}$ \\
\hline
\end{tabular}

\begin{tabular}{|l|l|l|}
\hline \multicolumn{2}{|l|}{ Revision History } \\
\hline A & $\begin{array}{l}\text { Michael Gibbons } \\
\text { Sarah Ur }\end{array}$ & 120608 \\
\hline B & Tristin McGuffick & 140605 \\
\hline C & Shelby Kunz & 160601 \\
\hline D & Conor Hedigan & 171101 \\
\hline E & Gabriella Sugerman & 180707 \\
\hline
\end{tabular}




\section{APPENDIX H: PROTOCOLS FOR CELL CULTURE FOR CONFOCAL}

\section{MICROSCOPY}

Purpose: The purpose of this SOP is to guide the user in thawing into an 8-chamber slide for confocal microscopy

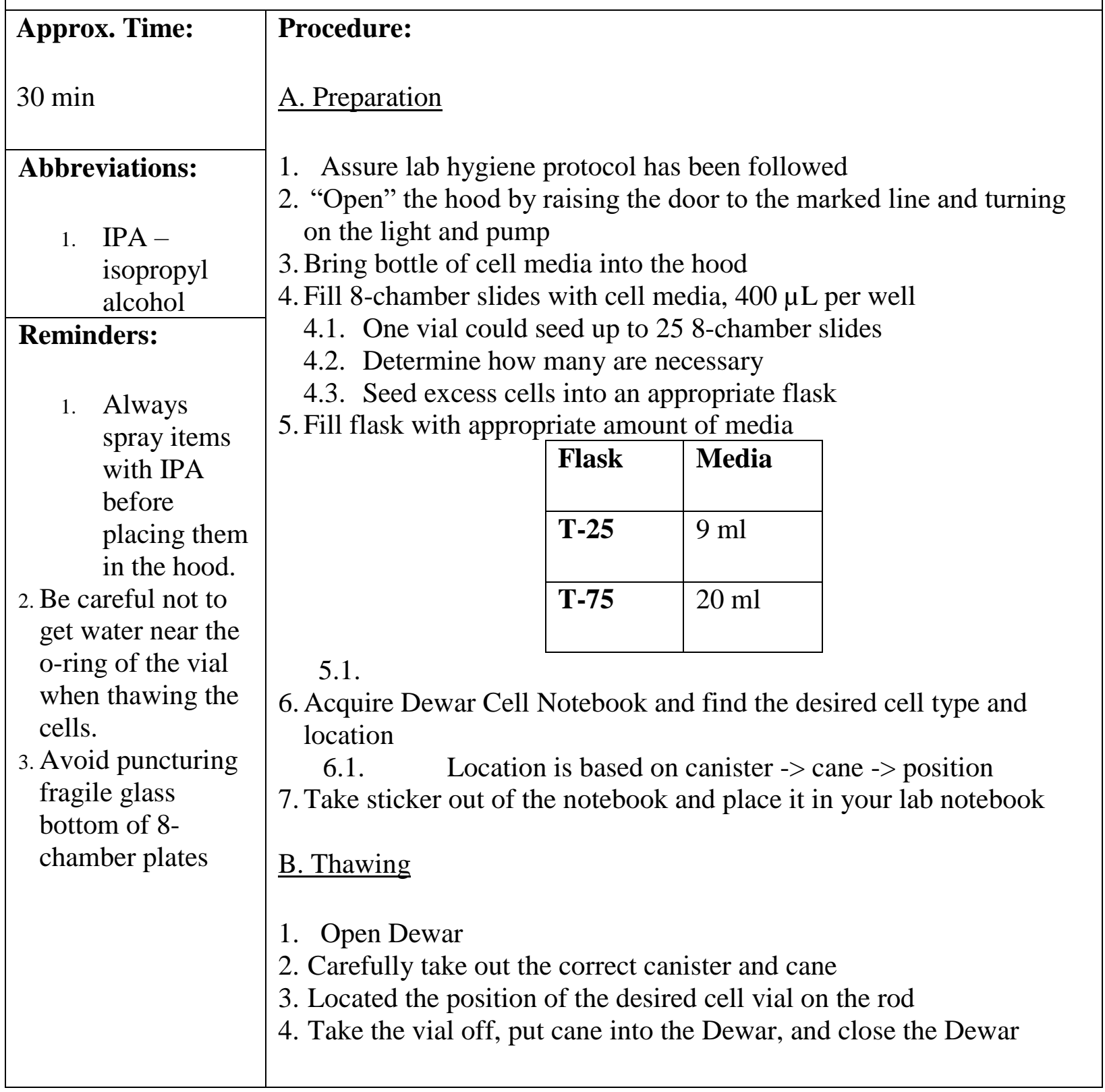




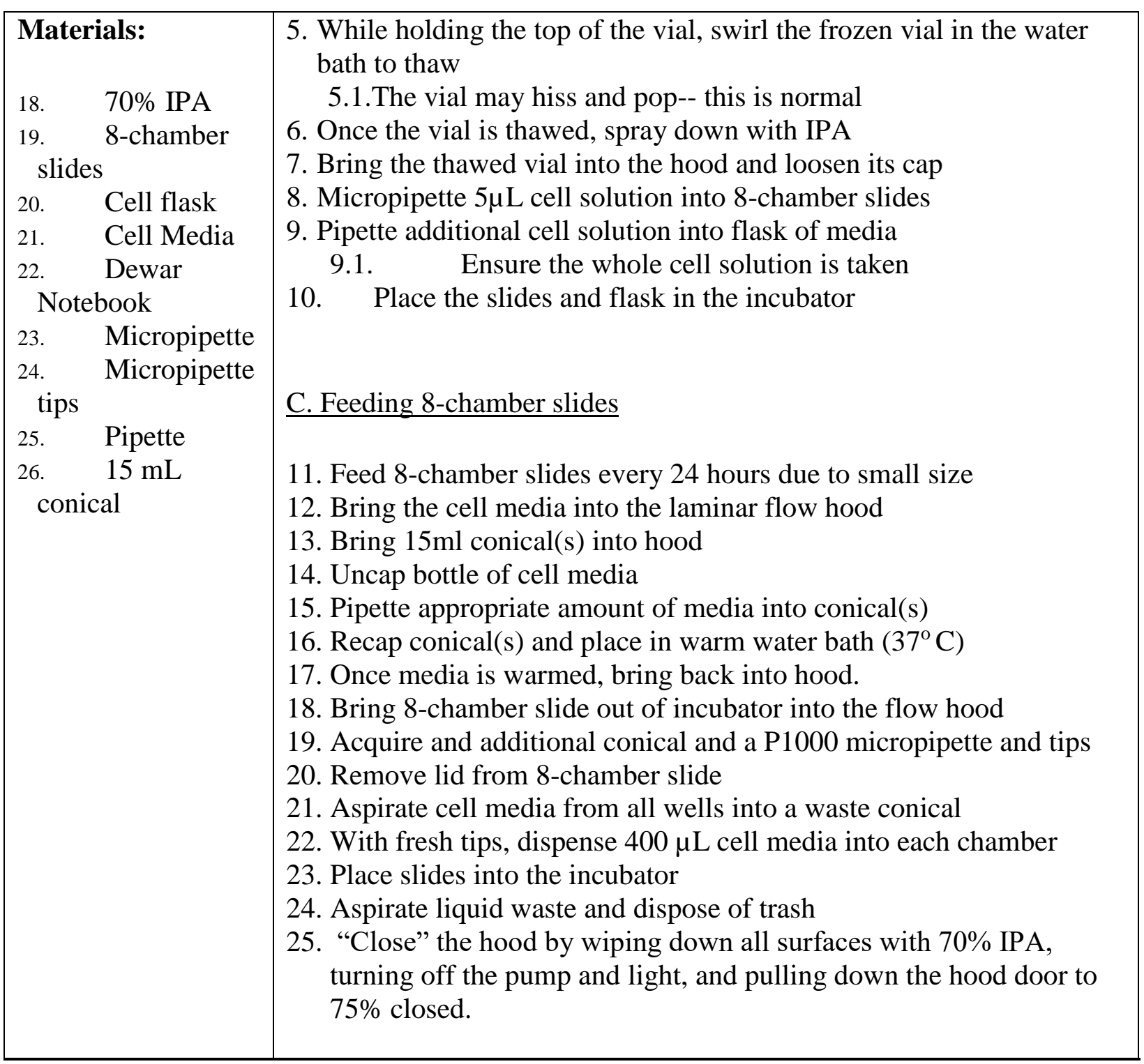

\begin{tabular}{|l|l|l|}
\hline \multicolumn{2}{|l|}{ Revision History: } \\
\hline A & Jakub Truty & Summer 2013 \\
\hline B & Gabriella Sugerman & 180708 \\
\hline & & \\
\hline
\end{tabular}


ASSESSMENT

Purpose: The purpose of this SOP is to guide the user in staining for ROS generation and viability assessment

Approx. Time:

3 hours

Abbreviations:

4. ROS:

Reactive

Oxygen

Species

5. DMSO:

dimethyl

sulfoxide

6. HBSS: Hank's

Buffered

Saline

Solution

7. TBHP: known

ROS inducer
Procedure:

A. Make Staining Solutions

1. Make $100 \mathrm{uM}$ TBHP solution

1.1 Add $1 \mu \mathrm{L}$ TBHP to $77 \mu \mathrm{L}$ water to make $100 \mathrm{mM}$ stock

1.2 Dilute stock 1:1000 in cell media

2. Make 5 uM Carboxy- $\mathrm{H}_{2}$ DCFDA solution

2.1 Combine $50 \mathrm{uL}$ DMSO with one vial powdered DCFDA and vortex to mix

2.2 Combine $10 \mathrm{uL}$ DCFDA with $4.0 \mathrm{~mL}$ HBSS

3. Make 2 uM EthD solution

3.1 Combine 5.1 uL $2 \mathrm{mM}$ EthD stock in $844.9 \mathrm{uL}$ HBSS

4. Make 1 uM Hoechst solution

4.1 Combine 10.8 uL Hoechst stock in 889.2 uL HBSS

B. TBHP Positive Control Treatment

1. Aspirate cell media from positive control samples

2. Apply $200 \mathrm{uL}$ of $100 \mathrm{uM}$ TBHP solution for 60 minutes 


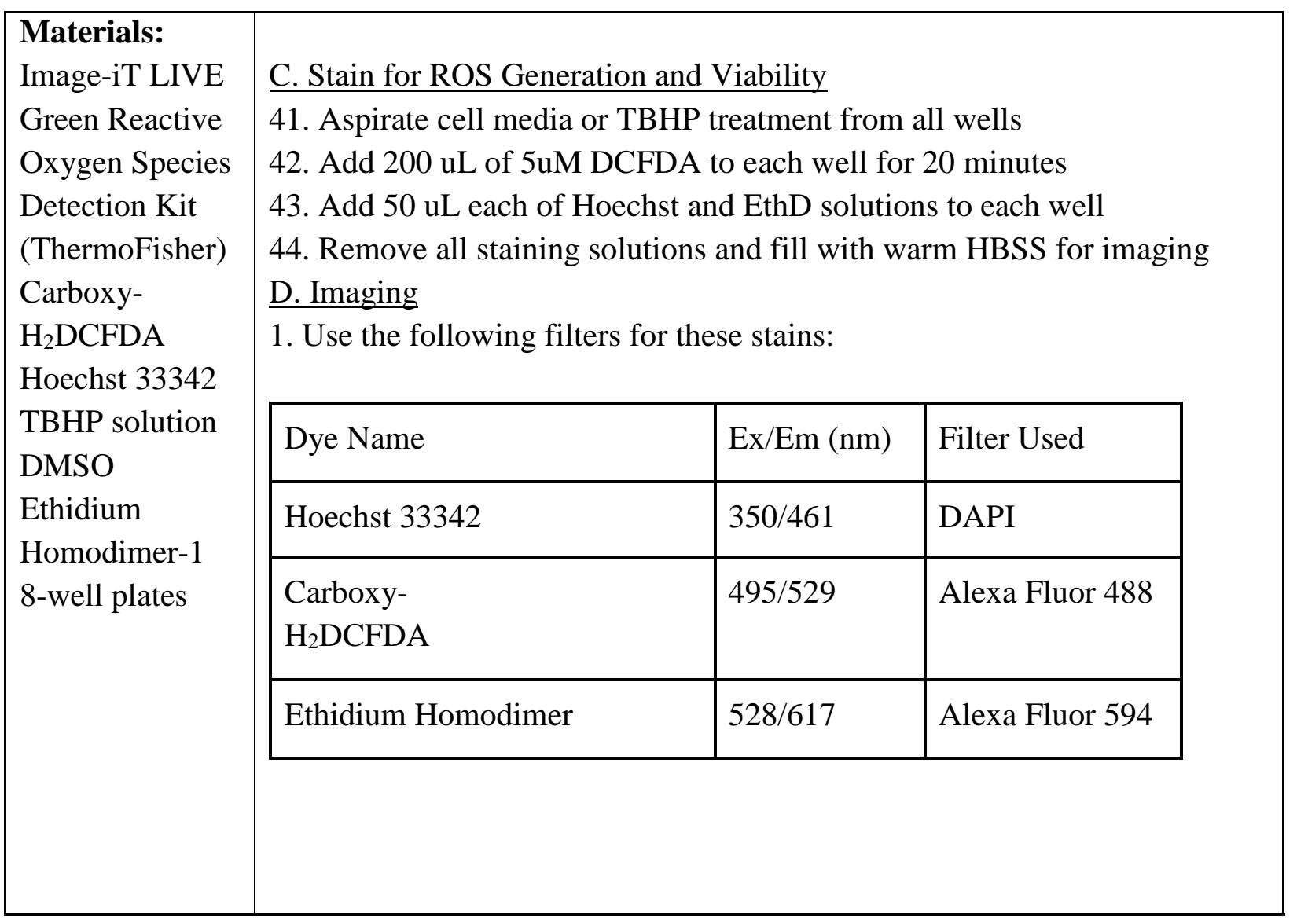

\begin{tabular}{|l|l|l|}
\hline Revision History: & Gabriella Sugerman & 180708 \\
\hline A & & \\
\hline & & \\
\hline
\end{tabular}




\section{APPENDIX J: PROTOCOLS FOR CONFOCAL IMAGING}

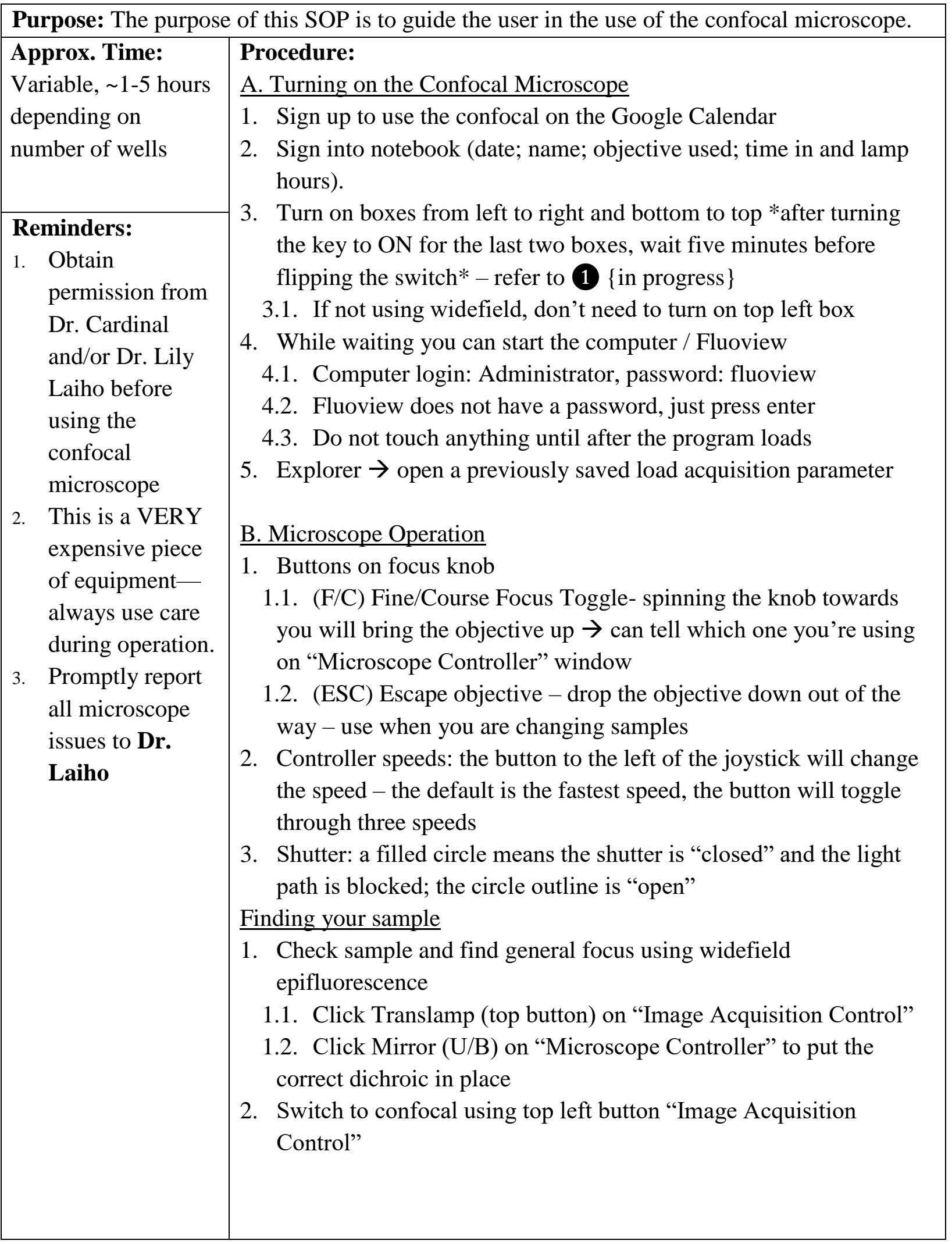




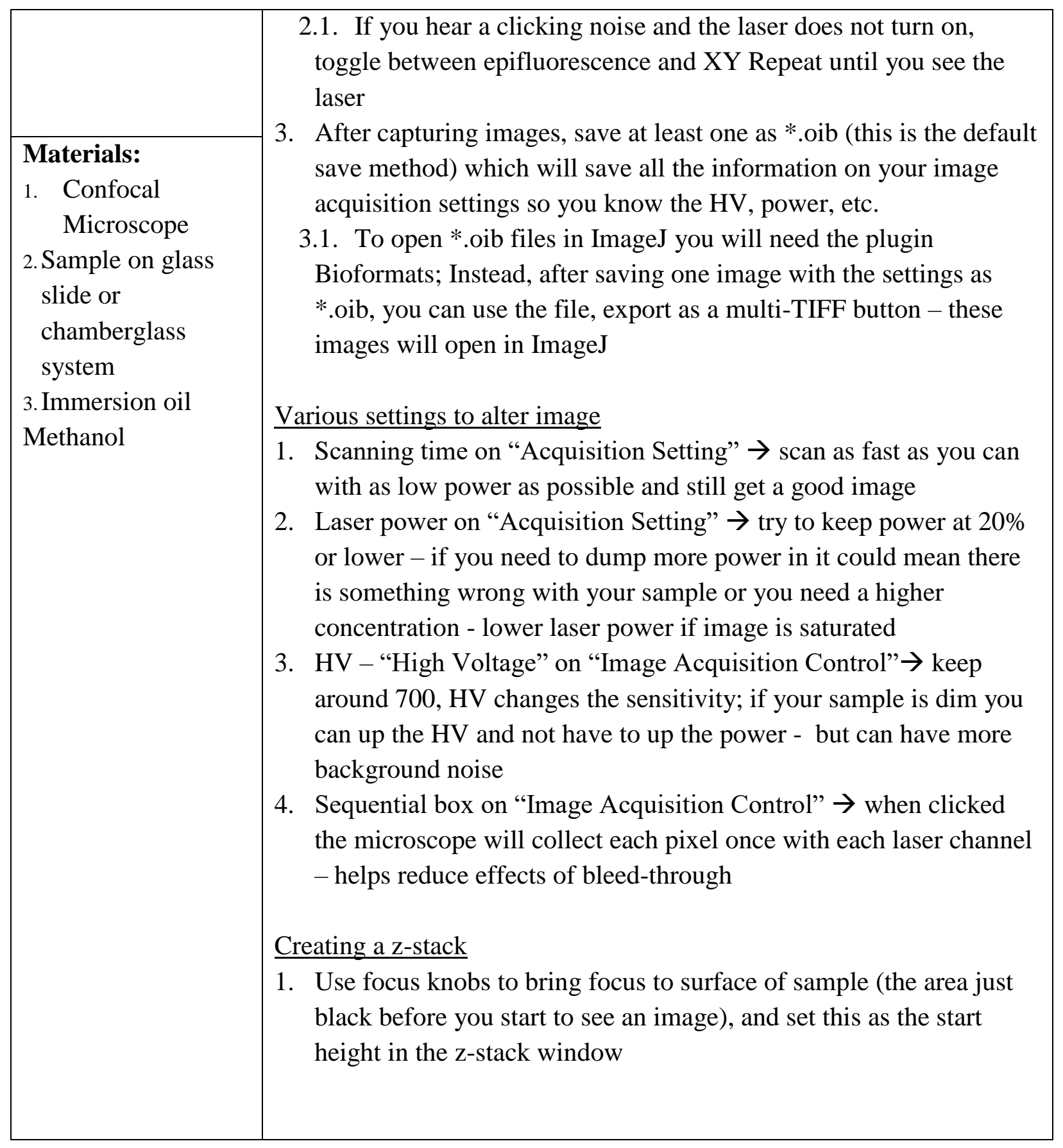




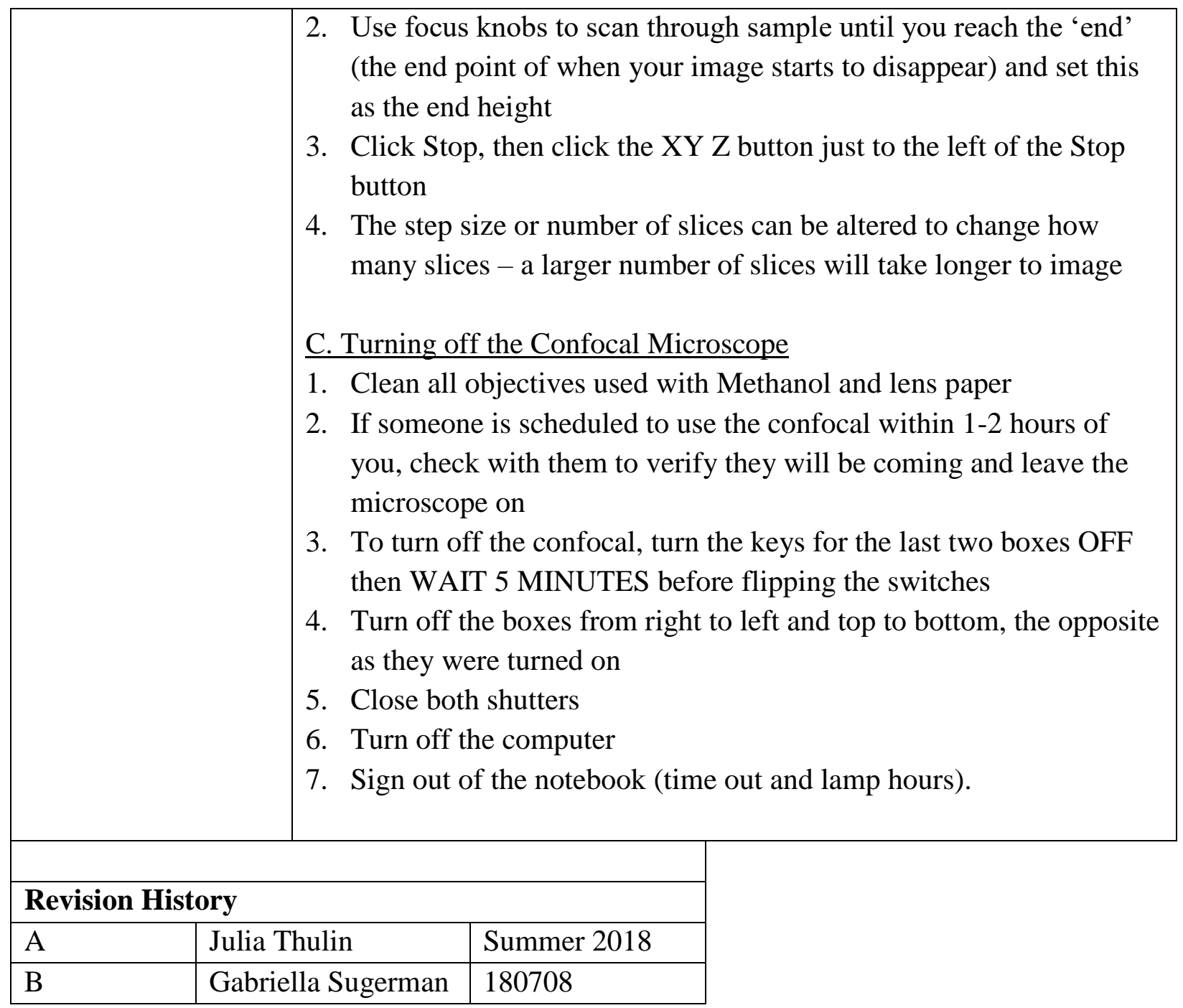




\section{APPENDIX K: MACROS FOR IMAGE PROCESSING}

Note: change "[YOUR FOLDER'S LOCATION]" to the path to where you want images saved, for example "C:/Desktop/My Images"

function split(inputDir, filename) \{

open(inputDir + filename);

run("Split Channels");

selectWindow("C1-" + filename);

saveAs("Tiff", "[YOUR FOLDER'S LOCATION]/C1-" + filename);

selectWindow("C2-" + filename);

saveAs("Tiff", "'[YOUR FOLDER’S LOCATION]/C2-" + filename);

selectWindow("C3-" + filename);

saveAs("Tiff", "[YOUR FOLDER'S LOCATION]/C3-" + filename);

close();

\}

setBatchMode(true);

inputDir = getDirectory("Choose input directory");

fileList $=$ getFileList(inputDir);

for $(\mathrm{i}=0 ; \mathrm{i}<$ fileList.length; $\mathrm{i}++)\{$

showProgress(i, fileList.length);

split(inputDir, fileList[i]);

\}

resultsDir = "[YOUR FOLDER'S LOCATION]" ;

function meangray(zProjectDir, maskDir, zProjectFile, maskFile) \{

open(maskDir + maskFile);

selectWindow(maskFile);

run("Analyze Particles...", "size=10-Infinity add");

open(zProjectDir + zProjectFile); 
selectWindow(zProjectFile);

roiManager("Select", Array.getSequence(roiManager("count")));

roiManager("Measure");

saveAs("Results", resultsDir + maskFile + "Results.csv");

Table.deleteRows(0, Table.size);

\}

setBatchMode(true);

zProjectDir = getDirectory ("Z Projections input directory");

maskDir = getDirectory("Masks input directory");

zFileList $=$ getFileList $(\mathrm{zProjectDir})$;

maskFileList $=$ getFileList $($ maskDir $)$;

for $(\mathrm{i}=0$; $\mathrm{i}<\mathrm{zFileList.length;} \mathrm{i++)} \mathrm{\{}$

meangray(zProjectDir, maskDir, zFileList[i], maskFileList[i]);

\} 


\section{APPENDIX L: ANOVA REPORTS}

qPCR

IL-6 Day 7

Fit Group

Oneway Analysis of CT By Column 1

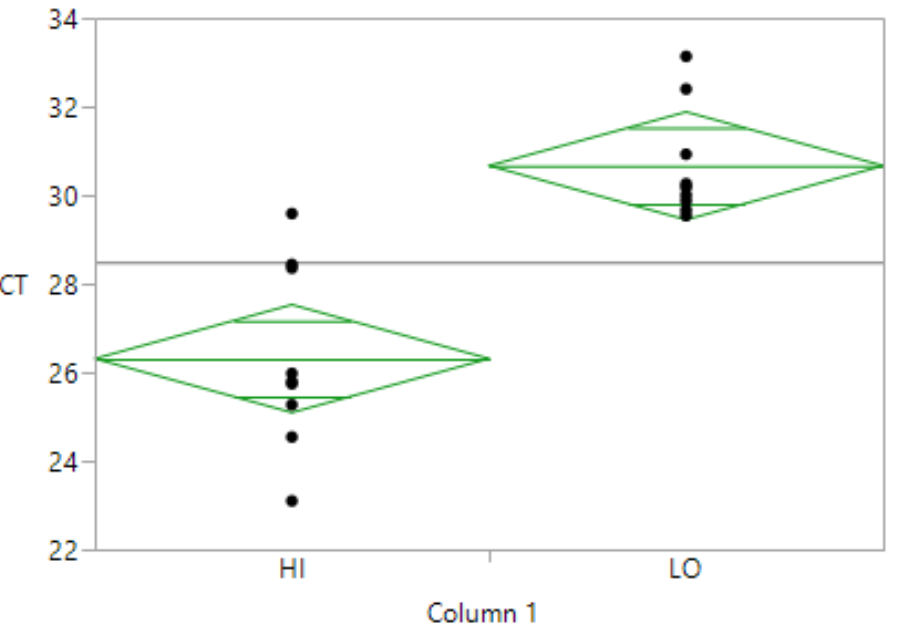

\begin{tabular}{|c|c|}
\hline \multicolumn{2}{|l|}{ Oneway Anova } \\
\hline \multicolumn{2}{|l|}{ Summary of Fit } \\
\hline Rsquare & 0.64201 \\
\hline Adj Rsquare & 0.619636 \\
\hline Root Mean Square Error & 1.724042 \\
\hline Mean of Response & 28.48894 \\
\hline Observations (or Sum Wgts) & 18 \\
\hline
\end{tabular}

t Test

LO-HI

Assuming equal variances

Difference $\quad 4.35349$ t Ratio $\quad 5.356682$

$\begin{array}{lll}\text { Std Err Dif } & 0.81272 \text { DF } & 16\end{array}$

Upper CL Dif 6.07638 Prob $>|t| \quad<.0001^{*}$

Lower CL Dif 2.63060 Prob $>t \quad<, 0001^{\star}$

Confidence $\quad 0.95$ Prob $<\mathrm{t} \quad 1.0000$

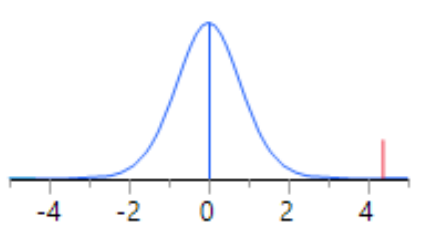

Analysis of Variance

\begin{tabular}{|c|c|c|c|c|c|}
\hline Source & DF & $\begin{array}{l}\text { Sum of } \\
\text { Squares }\end{array}$ & Mean Square & F Ratio & Prob $>F$ \\
\hline Column 1 & 1 & 85.28789 & 85.2879 & 28.6940 & $<.0001^{\star}$ \\
\hline Error & 16 & 47.55713 & 2.9723 & & \\
\hline C. Total & 17 & 132.84502 & & & \\
\hline
\end{tabular}

Means for Oneway Anova

Level Number Mean Std Error Lower 95\% Upper 95\%

$\begin{array}{llllll}\mathrm{HI} & 9 & 26.3122 & 0.57468 & 25.094 & 27.530\end{array}$

$\begin{array}{llllll}\text { LO } & 9 & 30.6657 & 0.57468 & 29.447 & 31.884\end{array}$

Std Error uses a pooled estimate of error variance 


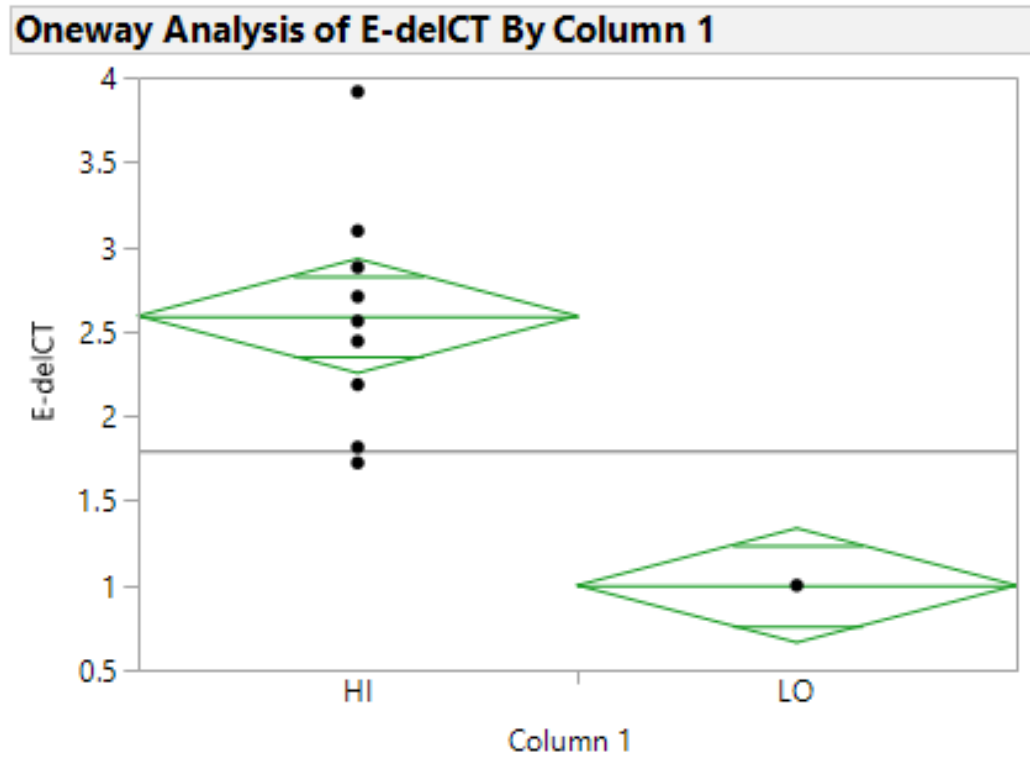

\begin{tabular}{|c|c|}
\hline \multicolumn{2}{|l|}{ Oneway Anova } \\
\hline \multicolumn{2}{|l|}{ Summary of Fit } \\
\hline Rsquare & 0.757673 \\
\hline Adj Rsquare & 0.742528 \\
\hline Root Mean Square Error & 0.477173 \\
\hline Mean of Response & 1.795499 \\
\hline Observations (or Sum Wgts) & 18 \\
\hline
\end{tabular}

\section{t Test}

LO-HI

Assuming equal variances

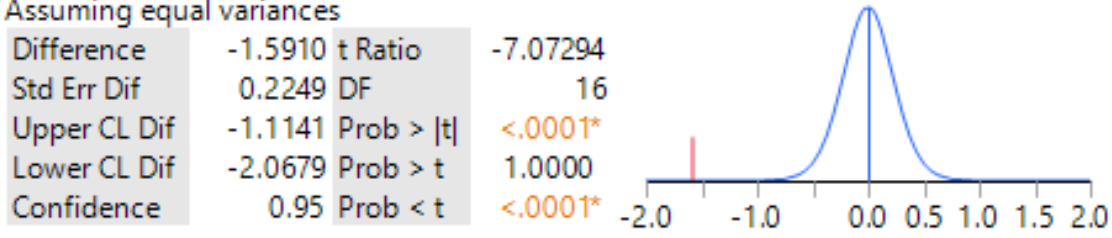

\begin{tabular}{|c|c|c|c|c|c|}
\hline \multicolumn{6}{|c|}{ Analysis of Variance } \\
\hline Source & DF & $\begin{array}{l}\text { Sum of } \\
\text { Squares }\end{array}$ & Mean Square & F Ratio & Prob $>F$ \\
\hline Column 1 & 1 & 11.390730 & 11.3907 & 50.0265 & $<.0001^{\star}$ \\
\hline Error & 16 & 3.643102 & 0.2277 & & \\
\hline C. Total & 17 & 15.033832 & & & \\
\hline
\end{tabular}

\section{Means for Oneway Anova}

Level Number Mean Std Error Lower 95\% Upper 95\%

$\begin{array}{llllll}\text { HI } & 9 & 2.59100 & 0.15906 & 2.2538 & 2.9282 \\ \text { LO } & 9 & 1.00000 & 0.15906 & 0.6628 & 1.3372\end{array}$

Std Error uses a pooled estimate of error variance 


\section{IL-6 Day 14}

\section{Fit Group}

\section{Oneway Analysis of CT By Column 1}

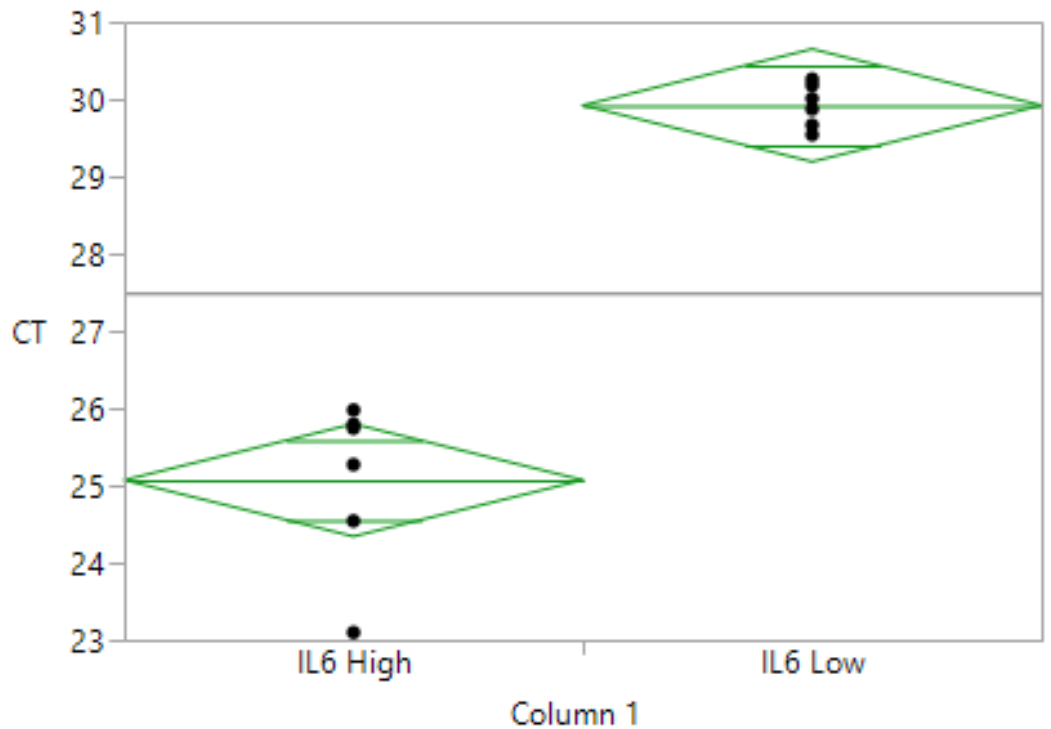

\begin{tabular}{|c|c|}
\hline Dneway Anova & \\
\hline Summary of Fit & \\
\hline Rsquare & 0.916712 \\
\hline Adj Rsquare & 0.908383 \\
\hline Root Mean Square Error & 0.800735 \\
\hline Mean of Response & 27.49662 \\
\hline Observations (or Sum Wgts) & 12 \\
\hline
\end{tabular}

\section{t Test}

IL6 Low-IL6 High

Assuming equal variances

Difference 4.85013 t Ratio $\quad 10.49121$

Std Err Dif

0.46230 DF

10

Upper CL Dif

5.88021 Prob $>|t|<.0001^{*}$

Lower CL Dif

3.82005 Prob $>t$

0.95 Prob $<\mathrm{t}$

$<, 0001^{*}$

1.0000

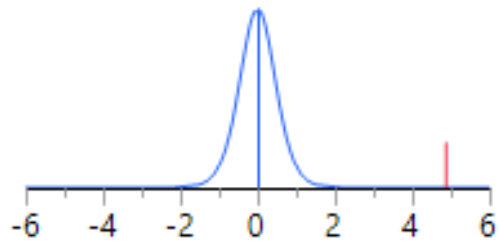

\section{Analysis of Variance}

\begin{tabular}{|c|c|c|c|c|c|}
\hline Source & DF & $\begin{array}{l}\text { Sum of } \\
\text { Squares }\end{array}$ & Mean Square & F Ratio & Prob $>F$ \\
\hline Column 1 & 1 & 70.571380 & 70.5714 & 110.0654 & $<.0001^{*}$ \\
\hline Error & 10 & 6.411768 & 0.6412 & & \\
\hline C. Total & 11 & 76.983148 & & & \\
\hline
\end{tabular}

\section{Means for Oneway Anova}

Level Number Mean Std Error Lower 95\% Upper 95\%

$\begin{array}{llllll}\text { IL6 High } & 6 & 25.0716 & 0.32690 & 24.343 & 25.800\end{array}$

$\begin{array}{lllll}\text { IL6 Low } \quad 6 & 29.9217 & 0.32690 & 29.193 & 30.650\end{array}$

Std Error uses a pooled estimate of error variance 


\section{Oneway Analysis of E-delCT By Column 1}

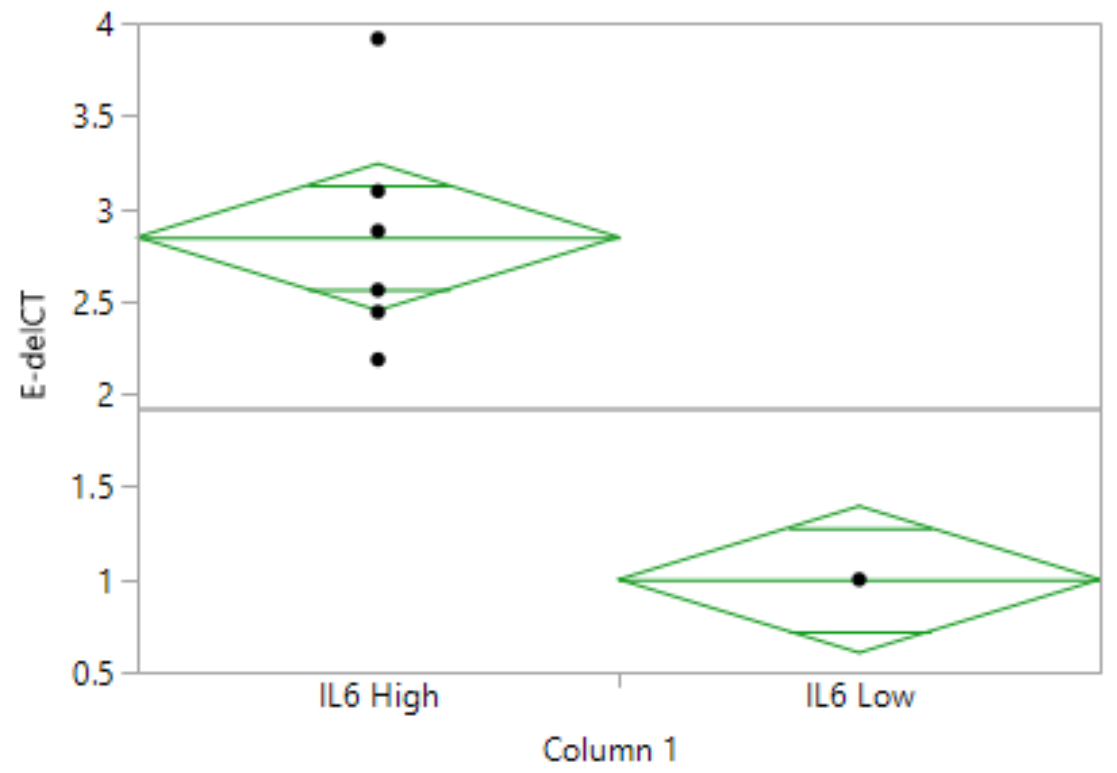

\section{Oneway Anova}

\section{Summary of Fit}

\begin{tabular}{lr}
\hline Rsquare & 0.844157 \\
Adj Rsquare & 0.828573 \\
Root Mean Square Error & 0.434383 \\
Mean of Response & 1.922892 \\
Observations (or Sum Wgts) & 12
\end{tabular}

\section{t Test}

IL6 Low-IL6 High

Assuming equal variances

Difference

-1.8458 t Ratio

$-7.35984$

Std Err Dif

Upper CL Dif

0.2508 DF

10

Lower CL Dif

-1.2870 Prob $>|t|$

-2.4046 Prob $>t$

Confidence

0.95 Prob $<\mathrm{t}$

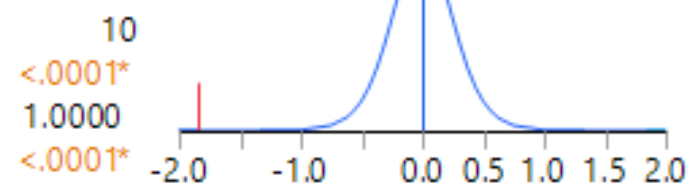

\section{Analysis of Variance}

\begin{tabular}{|c|c|c|c|c|c|}
\hline Source & DF & $\begin{array}{c}\text { Sum of } \\
\text { Squares }\end{array}$ & Mean Square & F Ratio & Prob $>F$ \\
\hline Column 1 & 1 & 10.220749 & 10.2207 & 54.1672 & $<.0001^{*}$ \\
\hline Error & 10 & 1.886890 & 0.1887 & & \\
\hline C. Total & 11 & 12.107638 & & & \\
\hline
\end{tabular}

\section{Means for Oneway Anova}

Level Number Mean Std Error Lower 95\% Upper 95\%

$\begin{array}{llllll}\text { IL6 High } & 6 & 2.84578 & 0.17734 & 2.4507 & 3.2409\end{array}$

IL6 Low $\quad 6 \quad 1.00000 \quad 0.17734 \quad 0.6049 \quad 1.3951$

Std Error uses a pooled estimate of error variance 


\section{Imaging}

\section{Fit Group}

Oneway Analysis of MGV By Duration

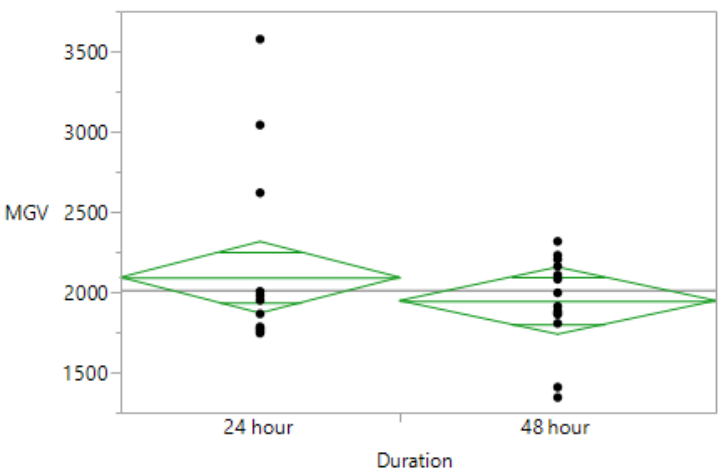

Oneway Anova

Summary of Fit

Adj Rsquare

Root Mean Square Error

Mean of Response

Observations (or Sum Wgts)

0.030353
-0.00197

422.3391

2015.569

32

t Test

48 hour-24 hour

Assuming equal variances

Difference $\quad-144.98$ t Ratio $\quad-0.96907$

Std Err Dif 149.61 DF

Upper CL Dif

Lower CL Dif

160.56 Prob $>|t|$

-450.53 Prob $>t$

0.95 Prob $<\mathrm{t} \quad 0.1701$

Confidence

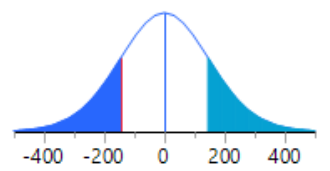

Analysis of Variance

\begin{tabular}{|c|c|c|c|c|c|}
\hline Source & DF & $\begin{array}{l}\text { Sum of } \\
\text { Squares }\end{array}$ & Mean Square & F Ratio & Prob $>F$ \\
\hline Duration & 1 & 167505.3 & 167505 & 0.9391 & 0.3403 \\
\hline Error & 30 & 5351109.9 & 178370 & & \\
\hline C. Total & 31 & 5518615.2 & & & \\
\hline
\end{tabular}

Means for Oneway Anova

Level Number Mean Std Error Lower 95\% Upper 95\%

\begin{tabular}{lrrrrr}
24 hour & 15 & 2092.59 & 109.05 & 1869.9 & 2315.3 \\
\hline
\end{tabular}

Std Error uses a pooled estimate of error variance

Oneway Analysis of Viability By Duration

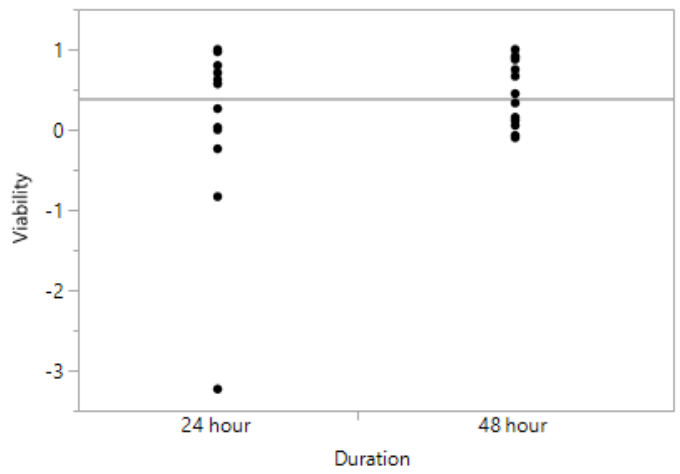

\section{Oneway Analysis of MGV By Treatment}

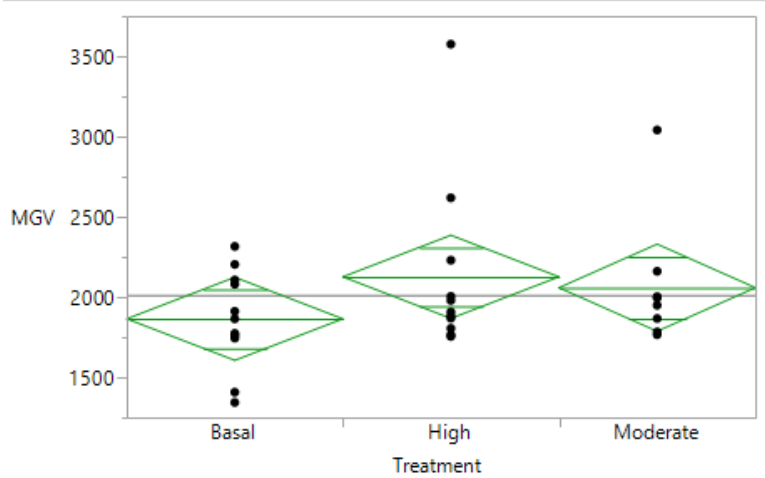

Oneway Anova

\section{Summary of Fit}

Rsquare

Adj Rsquare

0.073111

0.009187

Root Mean Square Error $\quad 419.9813$

Mean of Response 2015.569

Observations (or Sum Wgts) $\quad 32$

Analysis of Variance

\begin{tabular}{lrrrrr} 
& & \multicolumn{2}{c}{ Sum of } & & \\
Source & DF & Squares & Mean Square & F Ratio & Prob $>$ F \\
Treatment & 2 & 403470.4 & 201735 & 1.1437 & 0.3326 \\
Error & 29 & 5115144.8 & 176384 & & \\
C. Total & 31 & 5518615.2 & & &
\end{tabular}

\section{Means for Oneway Anova}

Level Number Mean Std Error Lower 95\% Upper 95\%

$\begin{array}{lrrrrr}\text { Basal } & 11 & 1865.13 & 126.63 & 1606.1 & 2124.1\end{array}$

\begin{tabular}{l|lllll} 
Basal & 11 & 1865.13 & 126.63 & 1606.1 & 2124.1 \\
High & 11 & 2126.67 & 126.63 & 1867.7 & 2385.7
\end{tabular}

$\begin{array}{llllll}\text { Moderate } & 10 & 2058.85 & 132.81 & 1787.2 & 2330.5\end{array}$

Std Error uses a pooled estimate of error variance

\section{Oneway Analysis of Viability By Treatment}

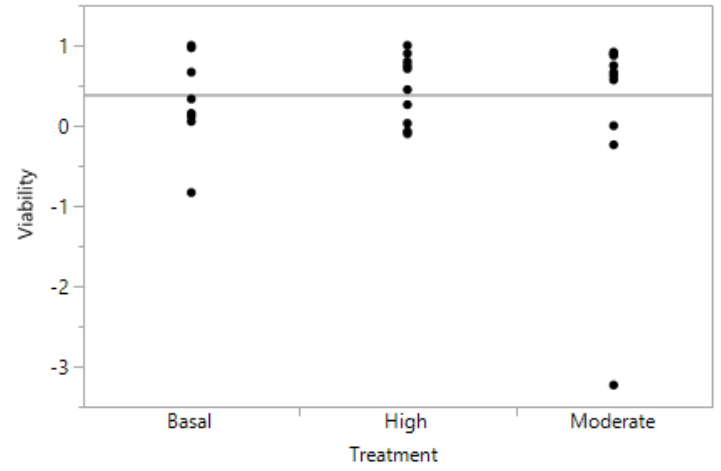




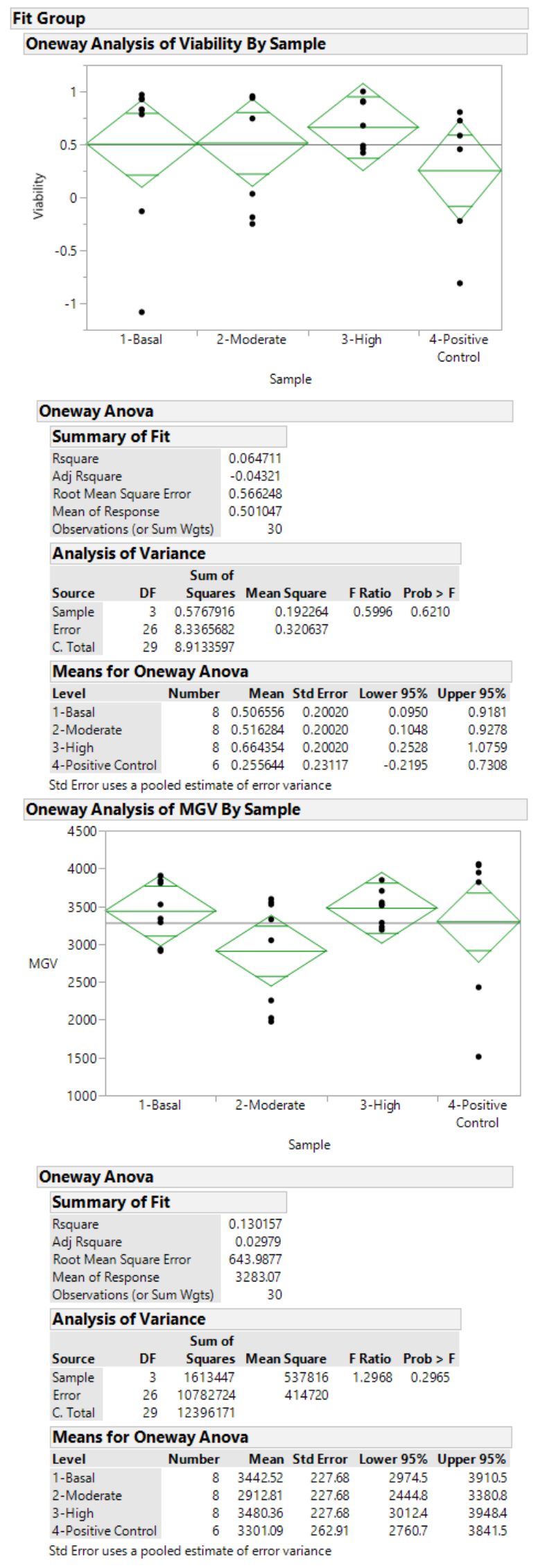




\section{APPENDIX M: RAW DATA}

\section{qPCR}

Testing Custom Primers

\begin{tabular}{|c|c|c|}
\hline Well & Sample Name & $\mathrm{Ct}$ \\
\hline E4 & ACTB & 30.1907 \\
\hline $\mathrm{F} 4$ & ACTB & 32.1067 \\
\hline G4 & ACTB & 30.1604 \\
\hline $\mathrm{H} 4$ & ACTB & Undetermined \\
\hline A4 & CD36-1 & Undetermined \\
\hline B4 & CD36-1 & Undetermined \\
\hline $\mathrm{C} 4$ & CD36-1 & Undetermined \\
\hline D4 & CD36-1 & Undetermined \\
\hline A3 & CD36-2 & Undetermined \\
\hline B3 & CD36-2 & Undetermined \\
\hline $\mathrm{C} 3$ & CD36-2 & Undetermined \\
\hline D3 & CD36-2 & 13.6893 \\
\hline A5 & CD68-1 & 31.9268 \\
\hline B5 & CD68-1 & 30.7794 \\
\hline C5 & CD68-1 & 30.4113 \\
\hline D5 & CD68-1 & Undetermined \\
\hline A6 & CD68-9 & Undetermined \\
\hline B6 & CD68-9 & Undetermined \\
\hline C6 & CD68-9 & Undetermined \\
\hline D6 & CD68-9 & Undetermined \\
\hline E3 & GAPDH & 21.3126 \\
\hline F3 & GAPDH & 20.5271 \\
\hline G3 & GAPDH & 20.2041 \\
\hline $\mathrm{H} 3$ & GAPDH & Undetermined \\
\hline A7 & IL1A-1 & Undetermined \\
\hline B7 & IL1A-1 & Undetermined \\
\hline C7 & IL1A-1 & Undetermined \\
\hline D7 & IL1A-1 & Undetermined \\
\hline A8 & IL1A-6 & 36.894 \\
\hline B8 & IL1A-6 & 35.4699 \\
\hline $\mathrm{C} 8$ & IL1A-6 & 35.2767 \\
\hline D8 & IL1A-6 & Undetermined \\
\hline A1 & IL6-2 & Undetermined \\
\hline
\end{tabular}




\begin{tabular}{|c|c|c|}
\hline B1 & IL6-2 & Undetermined \\
\hline $\mathrm{C} 1$ & IL6-2 & Undetermined \\
\hline D1 & IL6-2 & Undetermined \\
\hline A2 & IL6-8 & Undetermined \\
\hline B2 & IL6-8 & Undetermined \\
\hline $\mathrm{C} 2$ & IL6-8 & 29.1564 \\
\hline D2 & IL6-8 & Undetermined \\
\hline A9 & PLA2G7-1 & Undetermined \\
\hline B9 & PLA2G7-1 & Undetermined \\
\hline $\mathrm{C} 9$ & PLA2G7-1 & 39.4996 \\
\hline D9 & PLA2G7-1 & Undetermined \\
\hline A10 & PLA2G7-4 & Undetermined \\
\hline B10 & PLA2G7-4 & Undetermined \\
\hline $\mathrm{C} 10$ & PLA2G7-4 & Undetermined \\
\hline D10 & PLA2G7-4 & Undetermined \\
\hline A12 & PPARG-1 & 38.5619 \\
\hline B12 & PPARG-1 & 39.2674 \\
\hline $\mathrm{C} 12$ & PPARG-1 & 38.5588 \\
\hline D12 & PPARG-1 & Undetermined \\
\hline A11 & PPARG-6 & Undetermined \\
\hline B11 & PPARG-6 & Undetermined \\
\hline $\mathrm{C} 11$ & PPARG-6 & 37.2391 \\
\hline D11 & PPARG-6 & Undetermined \\
\hline $\mathrm{E} 2$ & PTGS2-1 & 27.7264 \\
\hline $\mathrm{F} 2$ & PTGS2-1 & 29.0332 \\
\hline $\mathrm{G} 2$ & PTGS2-1 & 27.5553 \\
\hline $\mathrm{H} 2$ & PTGS2-1 & Undetermined \\
\hline E1 & PTGS2-5 & 31.7588 \\
\hline $\mathrm{F} 1$ & PTGS2-5 & 32.4068 \\
\hline G1 & PTGS2-5 & 32.2364 \\
\hline $\mathrm{H} 1$ & PTGS2-5 & Undetermined \\
\hline
\end{tabular}

Standard Curves

\begin{tabular}{|r|l|l|}
\hline Dilution & Sample Name & Ct \\
\hline 0 & B2M & NTC \\
\hline 0 & CD36 & NTC \\
\hline 0 & HPRT1 & NTC \\
\hline 0 & IL6 & NTC \\
\hline
\end{tabular}




\begin{tabular}{|r|l|r|}
\hline 0.125 & B2M & 23.0772 \\
\hline 0.125 & B2M & 27.6819 \\
\hline 0.125 & B2M & 26.4787 \\
\hline 0.125 & CD36 & 36.1385 \\
\hline 0.125 & CD36 & 36.6827 \\
\hline 0.125 & CD36 & 36.6919 \\
\hline 0.125 & HPRT1 & 29.2113 \\
\hline 0.125 & HPRT1 & 29.6163 \\
\hline 0.125 & IL6 & 38.1149 \\
\hline 0.125 & IL6 & 36.5813 \\
\hline 0.125 & IL6 & 37.1748 \\
\hline 0.25 & B2M & 21.5211 \\
\hline 0.25 & B2M & 21.1977 \\
\hline 0.25 & B2M & 18.4869 \\
\hline 0.25 & CD36 & 29.6911 \\
\hline 0.25 & CD36 & 29.0239 \\
\hline 0.25 & CD36 & 28.3647 \\
\hline 0.25 & HPRT1 & 25.2433 \\
\hline 0.25 & HPRT1 & 24.5271 \\
\hline 0.25 & HPRT1 & 23.48 \\
\hline 0.25 & IL6 & 28.745 \\
\hline 0.25 & IL6 & 28.1088 \\
\hline 0.25 & IL6 & 30.802 \\
\hline 0.5 & B2M & 17.7577 \\
\hline 0.5 & B2M & 20.5211 \\
\hline 0.5 & B2M & 20.0036 \\
\hline 0.5 & CD36 & 27.8352 \\
\hline 0.5 & CD36 & 27.9685 \\
\hline 0.5 & HPRT1 & 24.2611 \\
\hline 0.5 & HPRT1 & 24.4024 \\
\hline 0.5 & HPRT1 & 27.0582 \\
\hline 0.5 & IL6 & 25.5383 \\
\hline 0.5 & IL6 & 19.3963 \\
\hline 0.5 & IL6 & 19.0834 \\
\hline 0.66 & B2M & \\
\hline 0.66 & B2M & \\
\hline 0.66 & B2M & \\
\hline 0.66 & CD36 & \\
\hline 0.66 & CD36 & \\
\hline & & 2424 \\
\hline
\end{tabular}




\begin{tabular}{|r|l|r|}
\hline 0.66 & CD36 & 27.7586 \\
\hline 0.66 & HPRT1 & 23.0095 \\
\hline 0.66 & HPRT1 & 22.9605 \\
\hline 0.66 & HPRT1 & 25.6776 \\
\hline 0.66 & IL6 & 26.9784 \\
\hline 0.66 & IL6 & 27.9516 \\
\hline 0.66 & IL6 & 28.0519 \\
\hline 1 & B2M & 18.6161 \\
\hline 1 & B2M & 18.66 \\
\hline 1 & B2M & 18.2691 \\
\hline 1 & CD36 & 25.5029 \\
\hline 1 & CD36 & 25.7805 \\
\hline 1 & CD36 & 25.352 \\
\hline 1 & HPRT1 & 21.7249 \\
\hline 1 & HPRT1 & 22.0442 \\
\hline 1 & HPRT1 & 21.8862 \\
\hline 1 & IL6 & 26.6202 \\
\hline 1 & IL6 & 27.0045 \\
\hline 1 & IL6 & 26.8122 \\
\hline
\end{tabular}

\section{B2M}

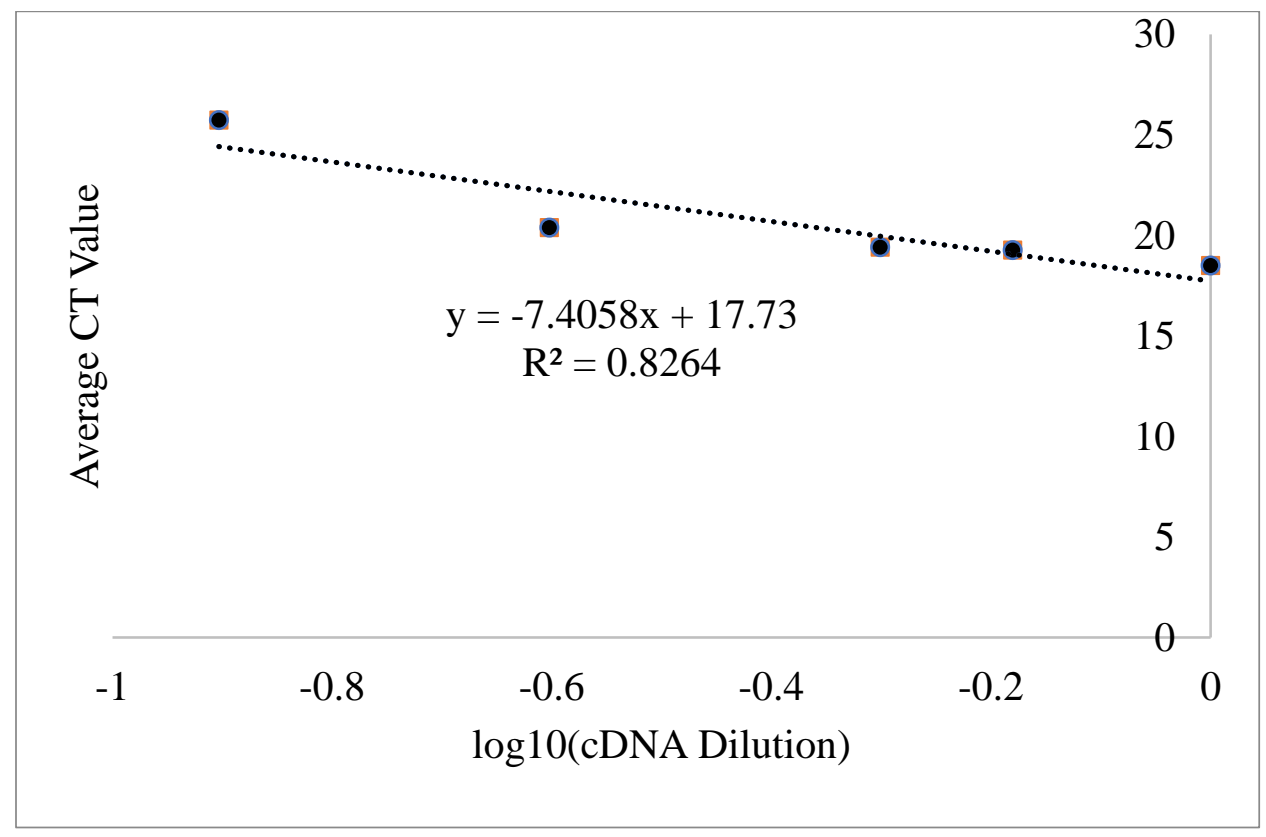


CD36

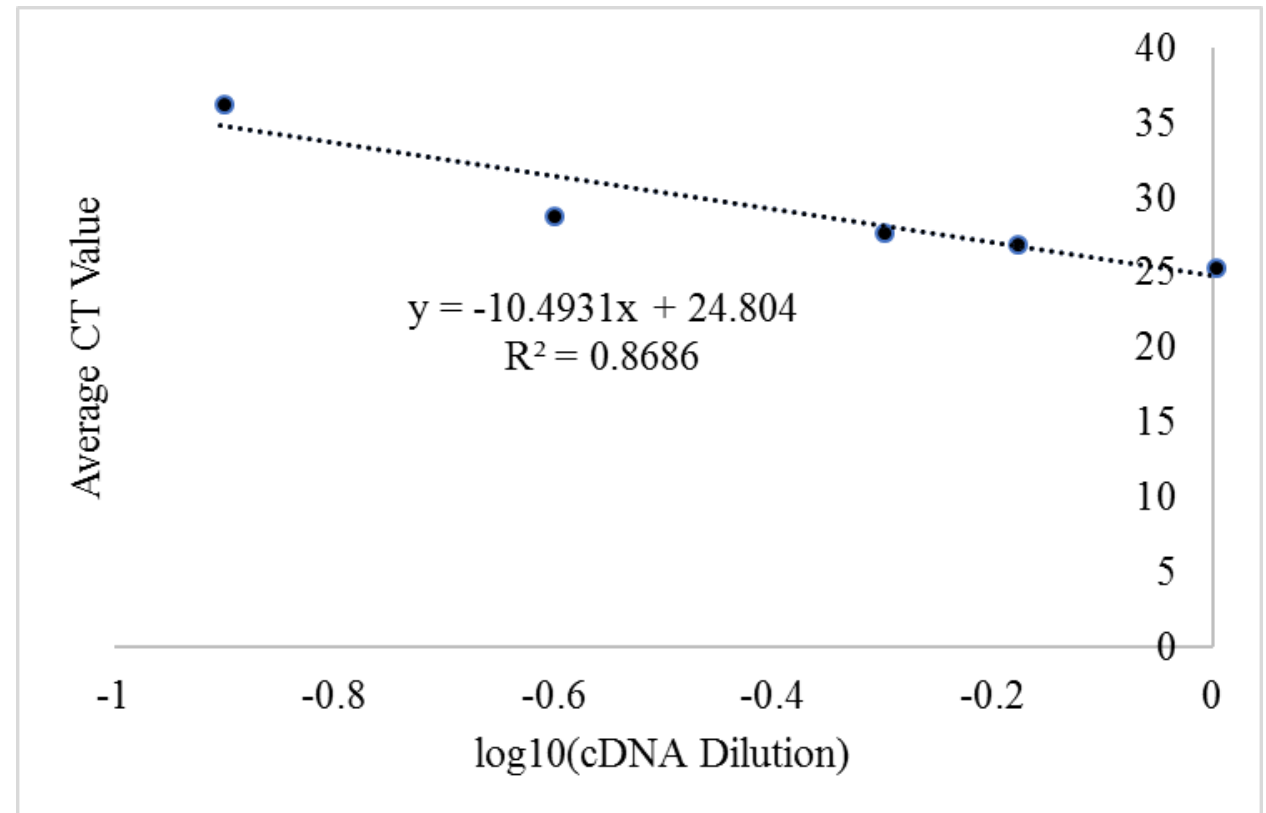

HPRT1

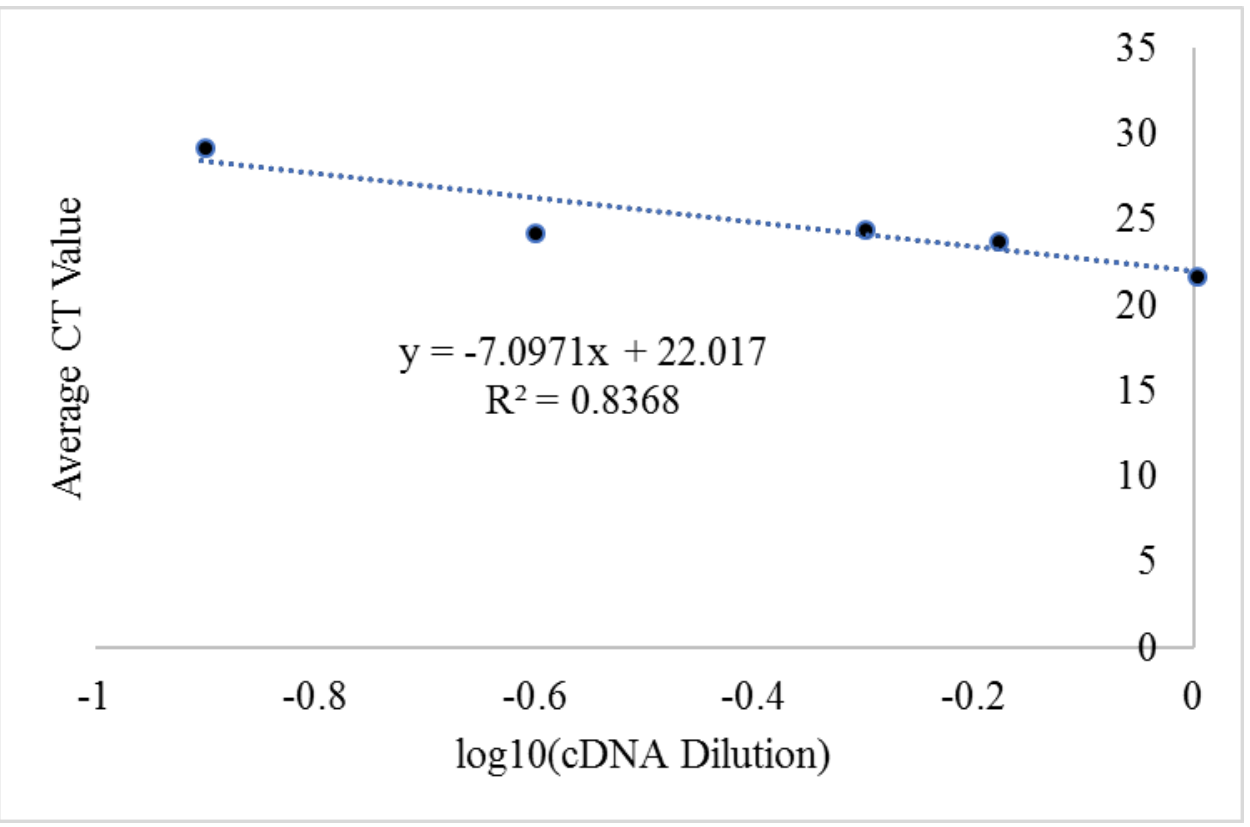


IL-6

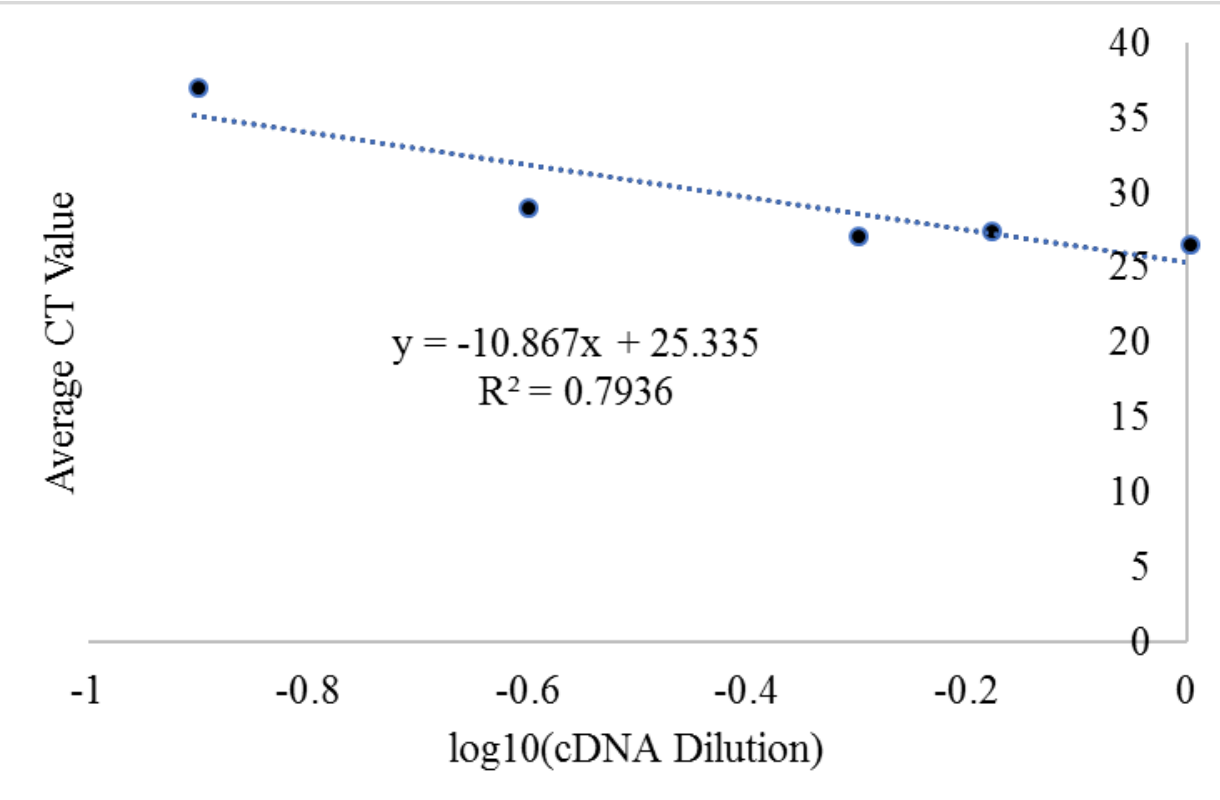

\begin{tabular}{|c|c|c|c|}
\hline 7-Day & & & \\
\hline Well & Treatment & Gene & $\mathrm{Ct}$ \\
\hline $\mathrm{C} 7$ & $\mathrm{HI}$ & $\mathrm{B} 2 \mathrm{M}$ & 16.5737 \\
\hline $\mathrm{C} 8$ & $\mathrm{HI}$ & $\mathrm{B} 2 \mathrm{M}$ & 15.4438 \\
\hline C9 & $\mathrm{HI}$ & B2M & 16.5884 \\
\hline C10 & $\mathrm{HI}$ & B2M & 18.1863 \\
\hline C11 & $\mathrm{HI}$ & B2M & 16.8739 \\
\hline $\mathrm{C} 12$ & $\mathrm{HI}$ & $\mathrm{B} 2 \mathrm{M}$ & 16.7503 \\
\hline A10 & LO & B2M & 17.039 \\
\hline A11 & LO & B2M & 15.8062 \\
\hline A12 & LO & B2M & 17.3592 \\
\hline $\mathrm{C} 1$ & LO & B2M & 27.5071 \\
\hline $\mathrm{C} 2$ & LO & $\mathrm{B} 2 \mathrm{M}$ & 27.2663 \\
\hline $\mathrm{C} 3$ & LO & $\mathrm{B} 2 \mathrm{M}$ & 22.8186 \\
\hline B10 & MED & B2M & 29.5639 \\
\hline B11 & MED & B2M & 30.457 \\
\hline B12 & MED & B2M & 30.9849 \\
\hline $\mathrm{C} 4$ & MED & $\mathrm{B} 2 \mathrm{M}$ & 29.8669 \\
\hline $\mathrm{C} 5$ & MED & $\mathrm{B} 2 \mathrm{M}$ & 29.4111 \\
\hline C6 & MED & B2M & 30.1312 \\
\hline C7 & HI & CD36 & 32.3459 \\
\hline C8 & $\mathrm{HI}$ & CD36 & 31.413 \\
\hline
\end{tabular}




\begin{tabular}{|l|l|l|r|} 
C9 & HI & CD36 & 32.3175 \\
\hline D7 & HI & CD36 & 32.7263 \\
\hline D8 & HI & CD36 & 33.8288 \\
\hline D9 & HI & CD36 & 32.8417 \\
\hline E7 & HI & CD36 & 34.3413 \\
\hline E8 & HI & CD36 & 34.3691 \\
\hline E9 & HI & CD36 & 34.096 \\
\hline C1 & LO & CD36 & 29.5586 \\
\hline C2 & LO & CD36 & 30.0567 \\
\hline C11 & LO & CD36 & 32.2382 \\
\hline D1 & LO & CD36 & 33.5947 \\
\hline D2 & LO & CD36 & 33.931 \\
\hline D3 & LO & CD36 & 34.1809 \\
\hline E1 & LO & CD36 & 33.7902 \\
\hline E2 & LO & CD36 & 34.321 \\
\hline E3 & LO & CD36 & 34.3671 \\
\hline C4 & MED & CD36 & 30.2788 \\
\hline C5 & MED & CD36 & 31.9086 \\
\hline C6 & MED & CD36 & 32.0858 \\
\hline D4 & MED & CD36 & 36.0872 \\
\hline D5 & MED & CD36 & 34.41 \\
\hline D6 & MED & CD36 & 35.0283 \\
\hline E4 & MED & CD36 & 37.4925 \\
\hline E5 & MED & CD36 & 35.3379 \\
\hline E6 & MED & CD36 & 35.4833 \\
\hline D7 & HI & HPRT1 & 25.2667 \\
\hline D8 & HI & HPRT1 & 25.7063 \\
\hline D9 & HI & HPRT1 & 24.3609 \\
\hline F7 & HI & HPRT1 & 23.7603 \\
\hline F8 & HI & HPRT1 & 23.6228 \\
\hline F9 & HI & HPRT1 & 24.5639 \\
\hline F10 & HI & HPRT1 & 23.016 \\
\hline F11 & HI & HPRT1 & 22.8775 \\
\hline F12 & HI & HPRT1 & 23.0758 \\
\hline D1 & LO & HPRT1 & 26.3175 \\
\hline D2 & LO & HPRT1 & 26.5942 \\
\hline D3 & LO & HPRT1 & 26.0351 \\
\hline D10 & LO & HPRT1 & 21.8116 \\
\hline D11 & LO & HPRT1 & 21.6623 \\
\hline & & & \\
\hline
\end{tabular}




\begin{tabular}{|l|l|l|r|}
\hline D12 & LO & HPRT1 & 21.9446 \\
\hline F1 & LO & HPRT1 & 23.9188 \\
\hline F2 & LO & HPRT1 & 24.032 \\
\hline F3 & LO & HPRT1 & 23.9561 \\
\hline D4 & MED & HPRT1 & 24.7127 \\
\hline D5 & MED & HPRT1 & 26.419 \\
\hline D6 & MED & HPRT1 & 29.6952 \\
\hline E10 & MED & HPRT1 & 34.5903 \\
\hline E11 & MED & HPRT1 & 34.2394 \\
\hline E12 & MED & HPRT1 & 37.4171 \\
\hline F4 & MED & HPRT1 & 37.9427 \\
\hline F5 & MED & HPRT1 & 33.974 \\
\hline F6 & MED & HPRT1 & 35.8646 \\
\hline E7 & HI & IL6 & 28.3562 \\
\hline E8 & HI & IL6 & 29.5847 \\
\hline E9 & HI & IL6 & 28.4396 \\
\hline G7 & HI & IL6 & 24.543 \\
\hline G8 & HI & IL6 & 25.2714 \\
\hline G9 & HI & IL6 & 25.7953 \\
\hline H7 & HI & IL6 & 25.7429 \\
\hline H8 & HI & IL6 & 25.9784 \\
\hline H9 & HI & IL6 & 23.0983 \\
\hline E1 & LO & IL6 & 30.9258 \\
\hline E2 & LO & IL6 & 32.3991 \\
\hline E3 & LO & IL6 & 33.1362 \\
\hline G1 & LO & IL6 & 29.8728 \\
\hline G2 & LO & IL6 & 30.2608 \\
\hline G3 & LO & IL6 & 30.0085 \\
\hline H1 & LO & IL6 & 30.1801 \\
\hline H2 & LO & IL6 & 29.6681 \\
\hline H3 & LO & IL6 & 29.5398 \\
\hline E4 & MED & IL6 & 32.808 \\
\hline E5 & MED & IL6 & 27.8487 \\
\hline G4 & MED & IL6 & 37.2573 \\
\hline G5 & MED & IL6 & 37.1969 \\
\hline G6 & MED & IL6 & 38.2392 \\
\hline H4 & MED & IL6 & 39.3774 \\
\hline H5 & MED & IL6 & 39.4759 \\
\hline H6 & MED & IL6 \\
\hline & & & \\
\hline
\end{tabular}


14-Day

\begin{tabular}{|l|l|l|}
\hline Well & $\begin{array}{l}\text { Sample } \\
\text { Name }\end{array}$ & \\
\hline C7 & B2M High & 16.501 \\
\hline C8 & B2M High & 15.3167 \\
\hline C9 & B2M High & 16.2626 \\
\hline C10 & B2M High & 18.2288 \\
\hline C11 & B2M High & 16.5286 \\
\hline C12 & B2M High & 16.5769 \\
\hline A10 & B2M Low & 17.2563 \\
\hline A11 & B2M Low & 15.6395 \\
\hline A12 & B2M Low & 17.5486 \\
\hline C1 & B2M Low & 27.8592 \\
\hline C2 & B2M Low & 27.8396 \\
\hline C3 & B2M Low & 22.143 \\
\hline B10 & B2M Med & 29.7833 \\
\hline B11 & B2M Med & 30.239 \\
\hline B12 & B2M Med & 30.4743 \\
\hline C4 & B2M Med & 29.7935 \\
\hline C5 & B2M Med & 29.5778 \\
\hline C6 & B2M Med & 30.0428 \\
\hline D7 & CD36 High & 32.0553 \\
\hline D8 & CD36 High & 33.6727 \\
\hline D9 & CD36 High & 32.2795 \\
\hline E7 & CD36 High & 34.1963 \\
\hline E8 & CD36 High & 34.844 \\
\hline E9 & CD36 High & 34.6044 \\
\hline D1 & CD36 Low & 33.3387 \\
\hline D2 & CD36 Low & 33.7992 \\
\hline D3 & CD36 Low & 34.7206 \\
\hline E1 & CD36 Low & 33.4278 \\
\hline E2 & CD36 Low & 34.1016 \\
\hline E3 & CD36 Low & 34.3213 \\
\hline D4 & CD36 Med & 36.2395 \\
\hline D5 & CD36 Med & 34.8253 \\
\hline D6 & CD36 Med & 35.7204 \\
\hline E4 & CD36 Med & 37.3185 \\
\hline E6 & 35.2208 \\
\hline
\end{tabular}




\begin{tabular}{|l|l|r|} 
F7 & HPRT1 High & 23.9428 \\
\hline F8 & HPRT1 High & 23.9193 \\
\hline F9 & HPRT1 High & 24.9483 \\
\hline F10 & HPRT1 High & 23.5463 \\
\hline F11 & HPRT1 High & 22.8958 \\
\hline F12 & HPRT1 High & 23.3137 \\
\hline D10 & HPRT1 Low & 21.0103 \\
\hline D11 & HPRT1 Low & 21.9891 \\
\hline D12 & HPRT1 Low & 21.7481 \\
\hline F1 & HPRT1 Low & 23.0031 \\
\hline F2 & HPRT1 Low & 24.6064 \\
\hline F3 & HPRT1 Low & 23.0133 \\
\hline E10 & HPRT1 Med & 34.0225 \\
\hline E11 & HPRT1 Med & 34.0841 \\
\hline E12 & HPRT1 Med & 37.8044 \\
\hline F4 & HPRT1 Med & 37.7632 \\
\hline F5 & HPRT1 Med & 33.3542 \\
\hline F6 & HPRT1 Med & 35.6349 \\
\hline G7 & IL6 High & 24.1783 \\
\hline G8 & IL6 High & 25.6371 \\
\hline G9 & IL6 High & 25.9949 \\
\hline H7 & IL6 High & 25.2269 \\
\hline H8 & IL6 High & 25.8689 \\
\hline H9 & IL6 High & 23.4803 \\
\hline G1 & IL6 Low & 29.0572 \\
\hline G2 & IL6 Low & 30.4029 \\
\hline G3 & IL6 Low & 30.3321 \\
\hline H1 & IL6 Low & 30.8583 \\
\hline H2 & IL6 Low & 29.5542 \\
\hline H3 & IL6 Low & 29.5898 \\
\hline G4 & IL6 Med & 37.47 \\
\hline G5 & IL6 Med & 37.7869 \\
\hline G6 & IL6 Med & 38.6494 \\
\hline H4 & IL6 Med & 39.7318 \\
\hline H5 & IL6 Med & 36.4187 \\
\hline H6 & IL6 Med & 39.5854 \\
\hline & & \\
\hline
\end{tabular}


Probe-Quencher Reporting

\begin{tabular}{|c|c|c|c|}
\hline Well & Sample Name & Detector & $\mathrm{Ct}$ \\
\hline A1 & ACTB Basal & FAM/IowaBlack & 32.5204 \\
\hline $\mathrm{A} 2$ & ACTB Basal & FAM/IowaBlack & 30.1749 \\
\hline $\mathrm{B} 1$ & ACTB Basal & FAM/IowaBlack & 34.6902 \\
\hline $\mathrm{B} 2$ & ACTB Basal & FAM/IowaBlack & 25.2126 \\
\hline B3 & ACTB Basal & FAM/IowaBlack & 33.6815 \\
\hline A8 & ACTB High & FAM/IowaBlack & 21.3877 \\
\hline B7 & ACTB High & FAM/IowaBlack & 15.6414 \\
\hline B8 & ACTB High & FAM/IowaBlack & 39.3752 \\
\hline A4 & ACTB Med & FAM/IowaBlack & 3.60218 \\
\hline B6 & ACTB Med & FAM/IowaBlack & 3.9693 \\
\hline $\mathrm{C} 1$ & B2M Basal & FAM/IowaBlack & 20.6675 \\
\hline $\mathrm{C} 2$ & B2M Basal & FAM/IowaBlack & 22.2837 \\
\hline $\mathrm{C} 3$ & B2M Basal & FAM/IowaBlack & 37.5111 \\
\hline D1 & B2M Basal & FAM/IowaBlack & 21.2299 \\
\hline $\mathrm{D} 2$ & B2M Basal & FAM/IowaBlack & 25.4048 \\
\hline C9 & B2M High & FAM/IowaBlack & 17.0982 \\
\hline D8 & B2M High & FAM/IowaBlack & 25.5236 \\
\hline D9 & B2M High & FAM/IowaBlack & 32.9716 \\
\hline $\mathrm{C} 5$ & B2M Med & FAM/IowaBlack & 19.0237 \\
\hline D5 & B2M Med & FAM/IowaBlack & 32.7524 \\
\hline $\mathrm{C} 11$ & CD36 High & FAM/IowaBlack & 26.0339 \\
\hline E7 & CD36 High & FAM/IowaBlack & 35 \\
\hline E9 & CD36 High & FAM/IowaBlack & 21.201 \\
\hline B10 & CD36 Med & FAM/IowaBlack & 17.6432 \\
\hline E5 & CD36 Med & FAM/IowaBlack & 17.0716 \\
\hline E6 & CD36 Med & FAM/IowaBlack & 34.2663 \\
\hline $\mathrm{H} 10$ & HPRT1 Basal & FAM/IowaBlack & 38.2972 \\
\hline D10 & HPRT1 Basal & FAM/IowaBlack & 29.0237 \\
\hline D12 & HPRT1 Basal & FAM/IowaBlack & 29.8932 \\
\hline $\mathrm{F} 2$ & HPRT1 Basal & FAM/IowaBlack & 31.091 \\
\hline F7 & HPRT1 High & FAM/IowaBlack & 29.156 \\
\hline F9 & HPRT1 High & FAM/IowaBlack & 20.2456 \\
\hline F11 & HPRT1 High & FAM/IowaBlack & 24.0113 \\
\hline F12 & HPRT1 High & FAM/IowaBlack & 29.3666 \\
\hline E10 & HPRT1 Med & FAM/IowaBlack & 20.8872 \\
\hline E12 & HPRT1 Med & FAM/IowaBlack & 31.5817 \\
\hline $\mathrm{F} 4$ & HPRT1 Med & FAM/IowaBlack & 26.6884 \\
\hline
\end{tabular}




\begin{tabular}{|l|l|l|r|} 
G1 & IL6 Basal & FAM/IowaBlack & 37.4332 \\
\hline G3 & IL6 Basal & FAM/IowaBlack & 7.89384 \\
\hline H1 & IL6 Basal & FAM/IowaBlack & 31.937 \\
\hline H3 & IL6 Basal & FAM/IowaBlack & 33.6775 \\
\hline G7 & IL6 High & FAM/IowaBlack & 35.3243 \\
\hline G9 & IL6 High & FAM/IowaBlack & 28.6016 \\
\hline H8 & IL6 High & FAM/IowaBlack & 28.953 \\
\hline G4 & IL6 Med & FAM/IowaBlack & 20.127 \\
\hline H4 & IL6 Med & FAM/IowaBlack & 27.9408 \\
\hline H5 & IL6 Med & FAM/IowaBlack & 26.0028 \\
\hline H6 & IL6 Med & FAM/IowaBlack & 26.1645 \\
\hline
\end{tabular}

\section{Image Analysis}

\section{Experiment 1}

\begin{tabular}{|l|l|r|r|r|r|}
\hline & & Count Hoechst & Count EthD & Count ROS & MGV ROS \\
\hline 48 hour & Basal & 6 & 8 & 0 & 1911.49 \\
\hline 48 hour & Basal & 9 & 1 & 6 & 1407.98 \\
\hline 48 hour & Basal & 9 & 8 & 3 & 2203.85 \\
\hline 24 hour & Moderate & 49 & 17 & 21 & 1949.77 \\
\hline 48 hour & Moderate & 12 & 13 & 1 & 1996.06 \\
\hline 24 hour & High & 10 & 9 & 2 & 3575.85 \\
\hline 24 hour & High & 31 & 8 & 9 & 2004.7 \\
\hline 48 hour & High & 10 & 11 & 1 & 2230.1 \\
\hline 24 hour & Basal & 34 & 34 & 1 & 1774.19 \\
\hline 24 hour & Moderate & 24 & 28 & 9 & 1783.68 \\
\hline 24 hour & High & 42 & 51 & 31 & 1979.34 \\
\hline 48 hour & Basal & 3 & 1 & 2 & 1343.84 \\
\hline 48 hour & Basal & 151 & 10 & 143 & 2315.86 \\
\hline 48 hour & Moderate & 15 & 19 & 5 & 1862.65 \\
\hline 48 hour & Moderate & 4 & 8 & 1 & 1867.15 \\
\hline 48 hour & High & 20 & 2 & 22 & 1805.47 \\
\hline 48 hour & High & 20 & 25 & 11 & 1868.65 \\
\hline 24 hour & Basal & 6 & 43 & 11 & 1864.96 \\
\hline 24 hour & Moderate & 21 & 90 & 26 & 2003.24 \\
\hline 24 hour & High & 68 & 101 & 66 & 2618.73 \\
\hline 48 hour & Basal & 13 & 13 & 11 & 2080.44 \\
\hline
\end{tabular}




\begin{tabular}{|l|l|r|r|r|r|}
48 hour & Basal & 17 & 14 & 15 & 2106.7 \\
\hline 48 hour & Moderate & 19 & 15 & 2 & 2161.07 \\
\hline 48 hour & Moderate & 32 & 13 & 4 & 2157.66 \\
\hline 48 hour & High & 14 & 6 & 15 & 1882.39 \\
\hline 48 hour & High & 8 & 11 & 2 & 1907.97 \\
\hline
\end{tabular}

\section{Experiment 2 Day 1 Raw}

\begin{tabular}{|c|c|c|c|c|}
\hline Stain & Treatment & Particle Count & Area & Mean Gray Value \\
\hline Hoechst & Basal & 103 & 31343.4 & 304.3052 \\
\hline Hoechst & Basal & 66 & 13219.8 & 200.3001 \\
\hline Hoechst & Basal & 94 & 20415.9 & 217.1903 \\
\hline Hoechst & Basal & 178 & 65772 & 369.5056 \\
\hline ROS & Basal & 7 & 8781.84 & 1254.5484 \\
\hline ROS & Basal & 9 & 3185.4 & 353.9336 \\
\hline ROS & Basal & 17 & 7462.94 & 438.9966 \\
\hline ROS & Basal & 61 & 29785.4 & 488.2859 \\
\hline EthD & Basal & 7 & 1072.08 & 153.1549 \\
\hline EthD & Basal & 2 & 410.323 & 205.1615 \\
\hline EthD & Basal & 7 & 1389.85 & 198.5505 \\
\hline EthD & Basal & 30 & 4314.56 & 143.8187 \\
\hline Hoechst & Moderate & 98 & 17967.8 & 183.3452 \\
\hline Hoechst & Moderate & 113 & 24239.9 & 214.5125 \\
\hline Hoechst & Moderate & 76 & 15544.5 & 204.5323 \\
\hline Hoechst & Moderate & 115 & 23434.7 & 203.7799 \\
\hline ROS & Moderate & 40 & 10967.7 & 274.1914 \\
\hline ROS & Moderate & 30 & 14865.7 & 495.5242 \\
\hline ROS & Moderate & 7 & 1318.9 & 188.4136 \\
\hline ROS & Moderate & 6 & 1366.72 & 227.7858 \\
\hline EthD & Moderate & 6 & 928.626 & 154.771 \\
\hline EthD & Moderate & 5 & 811.391 & 162.2781 \\
\hline EthD & Moderate & 4 & 735.805 & 183.9512 \\
\hline EthD & Moderate & 6 & 854.583 & 142.4304 \\
\hline Hoechst & High & 8 & 1611.98 & 201.4979 \\
\hline Hoechst & High & 19 & 3083.59 & 162.2944 \\
\hline Hoechst & High & 10 & 1610.44 & 161.0441 \\
\hline Hoechst & High & 88 & 17653.1 & 200.6039 \\
\hline ROS & High & 2 & 439.632 & 219.8159 \\
\hline
\end{tabular}




\begin{tabular}{|l|l|r|r|r|}
\hline ROS & High & 11 & 2730.34 & 248.2132 \\
\hline ROS & High & 51 & 18736 & 367.3731 \\
\hline ROS & High & 19 & 6273.62 & 330.1907 \\
\hline EthD & High & 0 & 0 & NaN \\
\hline EthD & High & 11 & 1679.86 & 152.7142 \\
\hline EthD & High & 1 & 112.607 & 112.6074 \\
\hline EthD & High & 8 & 1260.28 & 157.5347 \\
\hline
\end{tabular}

Experiment 2 Day 2 Raw

\begin{tabular}{|l|l|r|r|}
\hline Treatment & Stain & Particle Count & Mean Gray Value \\
\hline Positive Control & Hoechst & 126 & 5469.95 \\
\hline Positive Control & Hoechst & 4 & 111.065 \\
\hline Positive Control & Hoechst & 185 & 5724.47 \\
\hline Positive Control & Hoechst & 96 & 4965.53 \\
\hline Basal & Hoechst & 84 & 2204.33 \\
\hline Basal & Hoechst & 12 & 370.216 \\
\hline Basal & Hoechst & 154 & 14387.5 \\
\hline Basal & Hoechst & 480 & 66791.6 \\
\hline Moderate & Hoechst & 12 & 183.566 \\
\hline Moderate & Hoechst & 30 & 1470.07 \\
\hline Moderate & Hoechst & 662 & 63249.9 \\
\hline Moderate & Hoechst & 16 & 504.42 \\
\hline High & Hoechst & 201 & 19001.3 \\
\hline High & Hoechst & 645 & 33191.4 \\
\hline High & Hoechst & 102 & 10671.5 \\
\hline High & Hoechst & 239 & 22132.8 \\
\hline Positive Control & EthD & 154 & 4988.66 \\
\hline Positive Control & EthD & 8 & 175.853 \\
\hline Positive Control & EthD & 335 & 9346.42 \\
\hline Positive Control & EthD & 40 & 1004.21 \\
\hline Basal & EthD & 95 & 2869.18 \\
\hline Basal & EthD & 25 & 965.647 \\
\hline Basal & EthD & 27 & 939.424 \\
\hline Basal & EthD & 104 & 3154.55 \\
\hline Moderate & EthD & 15 & 504.42 \\
\hline Moderate & EthD & 29 & 933.254 \\
\hline Moderate & EthD & 169 & 5161.43 \\
\hline Moderate & EthD & 109 & 968.733 \\
\hline High & EthD & 2987.95 \\
\hline
\end{tabular}




\begin{tabular}{|l|l|r|r|} 
High & EthD & 330 & 9526.9 \\
\hline High & EthD & 55 & 1806.35 \\
\hline High & EthD & 77 & 2429.54 \\
\hline Positive Control & ROS & 25 & 13696.5 \\
\hline Positive Control & ROS & 24 & 21140.9 \\
\hline Positive Control & ROS & 4 & 447.345 \\
\hline Basal & ROS & 7 & 8781.84 \\
\hline Basal & ROS & 9 & 3185.4 \\
\hline Basal & ROS & 17 & 7462.94 \\
\hline Basal & ROS & 61 & 29785.4 \\
\hline Moderate & ROS & 40 & 10967.7 \\
\hline Moderate & ROS & 30 & 14865.7 \\
\hline Moderate & ROS & 7 & 1318.9 \\
\hline Moderate & ROS & 6 & 1366.72 \\
\hline High & ROS & 2 & 439.632 \\
\hline High & ROS & 11 & 2730.34 \\
\hline High & ROS & 51 & 18736 \\
\hline High & ROS & 19 & 6273.62 \\
\hline
\end{tabular}

